\title{
A New Table of Constant Weight Codes
}

\author{
A. E. BROUWER, JAMĖS B. SHEARER, N. J. A. SLOANE, FELl@w, IEEE, AND WARREN D. SMITH
}

\begin{abstract}
A table of binary constant weight codes of length $n \leq 28$ is presented. Explicit constructions are given for most of the 600 codes in the table; the majority of these codes are new. The known techniques for constructing constant weight codes are surveyed, and also a tablc is given of (unrestricted) binary codes of length $n \leq 28$.
\end{abstract}

\section{INTRODUCTION}

$\mathrm{T}$ HE MAIN GOAL of this paper is to givc an extensive tablc of lower bounds on $A(n, d, w)$, the maximal possible number of binary vectors of length $n$, Hamming distance at least $d$ apart, and constant weight $w$. We also give a table of lower bounds on $A(n, d)$, the maximal possible number of binary vectors of length $n$ and Hamming distance at least $d$ apart (with no restriction on weight).

Thesc functions have been studied by many authors, and were tabulated for $n \leq 24$ in [13], [45], [72], [132]. In the present paper we extend the tables to length $n \leq 28$.

Our main concern is with Table I, the table of constant weight codes. The majority of the 600 codes in this table are new, either because we have discovered nicer versions of existing codes, or (more frequently) because we have found better codes than were known before.

Our goal has been to give either an explicit construction or a reference for every code in the table. With some exceptions a rcadcr should be able to reconstruct any of these codes from the information given here. (This is in contrast to [13], where several codes are simply describcd as being found by an unstatcd "miscellaneous construction".) However, because of space limitations, we have not included explicit listings for the codes constructed in Section XII (indicated by " $y$ " in Tablc I) when they contain more than 1500 codewords.

Although [13] gives both upper and lower bounds on $A(n, d, w)$ and $A(n, d)$, in the present paper we give only lower bounds, i.e. tables of actual codes. We have not

Manuscript received August 26, 1989; revised February 21, 1990.

A. E. Brouwer is with the Department of Mathematics and Computer Science, Technological University of Eindhoven, $5600 \mathrm{MB}$ Eindhoven. Netherlands. Electronic mail: aeb(cwi.n1.

I. B. Shearer is with the Department of Mathematical Sciences, IBM Thomas J. Watson Research C.nter, P.O. Box 218, Yorktown Heights NY 10598. Elcctronic mail: jbs a ibm.com

N. I. A. Sloane is with the Mathcmatical Sciences Research Center, AT\&T Bell Laboratorics, Murray Hill, NI 07974. Electronic mail: useramhuxo.att.com

W. D. Smith was with the Mathematical Sciences Research Center, $\Lambda T \&$ T Bell Laboratories, Murray Hill, NJ 07974. He is now with NLC Research Institute, 4 Independence Way, Princeton, NJ 08544. Electronic mail: wds(a gauss.att.com

IEEE Log Number 9036748. given upper bounds for several reasons: 1) their calculation is a separate investigation, requiring analytic as opposed to combinatorial methods, 2) all the upper bounds in [13] for $d=10$ should be rechecked (see the Errata section), 3) it is very difficult to check upper bounds found by others, ' and 4) the paper is long enough already. However, wc do mention the cases where we know that our lower bound is actually the exact value.

$A(n, d, w)$ and $A(n, d)$ are fundamental combinatorial quantities. They are also used in thc construction of codes for asymmetric channels [16], [39], [49]-[51], [90], [180], DC-free codes [15], [64], [175], and spherical codes [167].

We would appreciate hearing of any improvements to the tables. Plcase send them to N. J. A. Sloane, Room 2C-376, AT\&T Bell Labs, Murray Hill, NJ 07974, USA; electronic mail address user@mhuxo.att.com.

Notation: The following notation will be used throughout. $\mathbb{F}_{a}$ denotes the Galois field of order $q$, while $\mathbb{Z}_{m}$ denotes the integers modulo $m$. An $[n, k, d]$ code is a linear code with length $n$, dimension $k$ and minimal distance $d$ [132]. Bars indicate complements of sets or binary vectors.

To save space we have sometimes written vectors in hexadecimal, using $0=0000, \cdots, 9=1001, \mathrm{~A}=1010, \cdots$, $\mathrm{F}=1111$, usually omitting leading zeros (so the vectors are right-justified). Superscripts (for example in Table $\mathrm{XV}$ ) indicate the number of vectors in an orbit. Parenthescs inside a vector (for example in Tables XII-XIV) indicate that all simultaneous cyclic shifts of the parenthesized sections are to be used. For example $(110)(10)$ is an abbreviation for the six vectors $11010,01101,10110$, $11001,01110,10101$.

A design $\left(X, x^{\prime}\right)$ is a set $X$ (of "points") together with a collection $\mathscr{B}$ of subscts of $X$ (called "blocks"). A $t-(v, k, \lambda)$ design is a design in which $|X|=v$, all blocks contain exactly $k$ points, and any $t$ distinct points of $X$ belong to exactly $\lambda$ blocks ([14], [27], [31], [77], [96], [163], [176]). A Steiner system $S(t, k, v)$ is a $t-(v, k, 1)$ design. A balanced incomplete block design is a $2-(v, k, \lambda)$ design. More generally an $(r, \lambda)$-design is a design in which cach point bclongs to exactly $r$ blocks and each pair of points belong to exactly $\lambda$ blocks (but the blocks need not all contain the same number of points). A symmetric design

\footnotetext{
${ }^{1}$ An extreme case is the recent theorem of Lam et al. that there is no projective plane of order 10 [120]. This result, based on thousands of hours of computer time and as yet unchecked [141], implies $A(111,20,11) \leq 110$ (see $[132$, p. 528]).
} 
TABLE I-A

L.OWER Bocidos on $A(n, 4, w)$

\begin{tabular}{|c|c|c|c|c|c|c|c|c|c|c|c|c|}
\hline$n, w$ & $3^{23}$ & $4^{25}$ & 5 & 6 & 7 & 8 & 9 & 10 & 11 & 12 & 13 & 14 \\
\hline 6 & $4^{a}$ & 3 & 1 & 1 & 0 & 0 & 0 & 0 & 0 & 0 & 0 & 0 \\
\hline 7 & - $7 .^{s s}$ & 7 & 3 & 1 & 1 & 0 & 0 & 0 & 0 & 0 & 0 & 0 \\
\hline 8 & $.8^{c}$ & $14 .^{s s}$ & 8 & 4 & 1 & 1 & 0 & 0 & 0 & 0 & 0 & 0 \\
\hline 9 & $12 .^{s}$ & $18 .^{s}$ & 18 & 12 & 4 & 1 & 1 & 0 & 0 & 0 & 0 & 0 \\
\hline 10 & $13 \mathrm{~s}^{\mathrm{s}}$ & $30 .^{c}$ & $36 .^{s}$ & 30 & 13 & 5 & 1 & 1 & 0 & 0 & 0 & 0 \\
\hline 11 & $17 .^{s}$ & $35^{p c}$ & $66^{s}$ & 66 & 35 & 17 & 5 & 1 & 1 & 0 & 0 & 0 \\
\hline 12 & $20 .^{s}$ & $51 .^{p 0}$ & $80^{y}$ & $132 .^{s s}$ & 80 & 51 & 20 & 6 & 1 & 1 & 0 & 0 \\
\hline 13 & $26 .^{c}$ & $65 s^{s}$ & $123^{y}$ & $166^{y}$ & 166 & 123 & 65 & 26 & 6 & 1 & 1 & 0 \\
\hline 14 & $28^{s}$ & $91 .{ }^{* s}$ & $169^{8}$ & $278^{y a}$ & $325^{y d}$ & 278 & 169 & 91 & 28 & 7 & 1 & 1 \\
\hline 15 & $35 .^{s}$ & $105^{s}$ & $237^{\text {yd }}$ & $389^{y}$ & $585^{s}$ & 585 & 389 & 237 & 105 & 35 & 7 & 1 \\
\hline 16 & 37.5 & $140 .^{s s}$ & $312^{y d}$ & $615^{y}$ & $836^{\text {sd }}$ & $1170^{n}$ & 836 & 615 & 312 & 140 & 37 & 8 \\
\hline 17 & $44 .^{s}$ & $156^{e c}$ & $424^{p 0}$ & $854^{p !}$ & $1416^{5}$ & $1770^{s}$ & 1770 & 1416 & 854 & 424 & 156 & 44 \\
\hline 18 & $48 .^{s}$ & $198 .^{c}$ & $518^{y}$ & $1260^{\circ}$ & $2041^{p 3}$ & $3186^{s}$ & $3540^{s}$ & 3186 & 2041 & 1260 & 518 & 198 \\
\hline 19 & $57 . .^{c}$ & $228 .^{s}$ & $684^{s}$ & $1596^{s}$ & $3172^{p 1}$ & $4667^{p 0}$ & $6726^{s}$ & 6726 & 4667 & 3172 & 1596 & 684 \\
\hline 20 & $600^{s}$ & $285 .^{p 0}$ & $874^{y}$ & $2280^{8}$ & $4213^{\rho 0}$ & $7730^{\rho 0}$ & $10039^{00}$ & $13452^{6}$ & 10039 & 7730 & 4213 & 2280 \\
\hline 21 & $70 .^{s}$ & $315^{s}$ & $1071^{s}$ & $2856^{5}$ & $6120^{\circ 0}$ & $10726^{\rho 1}$ & $16856^{p^{0}}$ & $20188^{p 2}$ & 20188 & 16856 & 10726 & 6120 \\
\hline 22 & $73 . s$ & $385^{s}$ & $1386 .^{s}$ & $3927^{s}$ & $8211^{p 0}$ & $16354^{p 1}$ & $25570^{\circ 3}$ & $36381^{p^{2}}$ & $39688^{p 3}$ & 36381 & 25570 & 16354 \\
\hline 23 & $83 .^{5}$ & $416^{0}$ & $1771 .^{s}$ & $5313^{s}$ & $11638^{s}$ & $23276^{s}$ & $40786^{p 1}$ & $57436^{\circ 0}$ & $73794^{p 1}$ & 73794 & 57436 & 40786 \\
\hline 24 & $88^{s}$ & $498 p^{0 U}$ & $1895^{\circ} \bullet$ & 7084. ss & $15554^{p 0}$ & $34914^{8}$ & $59262^{p 0}$ & $96496^{p 0}$ & $116914^{p 0}$ & $146552^{p 0}$ & 116914 & 96496 \\
\hline 25 & $100^{c}$ & $5500^{s}$ & $2334^{p}$ & $7772^{\rho 1}$ & $21094^{p 0}$ & $46390^{p 1}$ & $88411^{p^{0}}$ & $140320^{\rho 0}$ & $194756^{p 0}$ & $227168^{p 0}$ & 227168 & 194756 \\
\hline 26 & $104 .^{c}$ & $650 .^{s s}$ & $2670^{s}$ & $10010^{p 1}$ & $26920^{p 0}$ & $65260^{p 1}$ & $128024^{p 0}$ & $218853^{p !}$ & $315648^{p 0}$ & $394874^{p 1}$ & $424868^{p 0}$ & 394874 \\
\hline 27 & $117 .^{s}$ & $702 . s$ & $3276^{s}$ & $12012^{s}$ & $35510^{p 1}$ & $87709^{0}$ & $184420^{p 1}$ & $329076^{p 0}$ & $506444^{\rho 1}$ & $672148^{p 0}$ & $774565^{p 1}$ & 774565 \\
\hline 28 & $121^{.3}$ & $819 .^{s s}$ & $3718^{p 0}$ & $15288^{8}$ & $44747^{p 0}$ & $121403^{p 0}$ & $259703^{p 0}$ & $502068^{p 0}$ & $806303^{p 0}$ & $1154541^{p 0}$ & $1399597^{p 0}$ & $1520224^{\rho 0}$ \\
\hline
\end{tabular}

TABLE I-B

LOWER BOUNDS ON $A(n, 6, w)$

\begin{tabular}{|c|c|c|c|c|c|c|c|c|c|c|c|}
\hline$n, w$ & $4^{24}$ & 5 & 6 & 7 & 8 & 9 & 10 & 11 & 12 & 13 & 14 \\
\hline 8 & 2. & 2 & 1 & 1 & 1 & 0 & 0 & 0 & 0 & 0 & 0 \\
\hline 9 & $3 .^{s}$ & 3 & 3 & 1 & 1 & 1 & 0 & 0 & 0 & 0 & 0 \\
\hline 10 & $5 .^{a}$ & $6 .^{s}$ & 5 & 3 & 1 & 1 & 1 & 0 & 0 & 0 & 0 \\
\hline 11 & $6 .^{s}$ & $11 .^{c}$ & 11 & 6 & 3 & 1 & 1 & 1 & 0 & 0 & 0 \\
\hline 12 & 9.s & $12 .^{c}$ & $22 .^{h m}$ & 12 & 9 & 4 & 1 & 1 & 1 & 0 & 0 \\
\hline 13 & $13 .^{c}$ & 18.5 & $26{ }^{\circ}$ & 26 & 18 & 13 & 4 & 1 & 1 & 1 & 0 \\
\hline 14 & $14 .^{c}$ & $28 .^{s}$ & $42 .^{c}$ & $42 .^{n n}$ & 42 & 28 & 14 & 4 & 1 & 1 & 1 \\
\hline 15 & $15^{c}$ & $42 s^{s}$ & $70 .^{s}$ & $69^{h 1}$ & 69 & 70 & 42 & 15 & 5 & 1 & 1 \\
\hline 16 & $20 .^{s}$ & $48 .^{s}$ & $112^{t:}$ & $109^{h 1}$ & $120^{t 1}$ & 109 & 112 & 48 & 20 & 5 & 1 \\
\hline 17 & 20. & $68 s^{s s}$ & 112 & $166^{h 1}$ & $184^{8}$ & 184 & 166 & 112 & 68 & 20 & 5 \\
\hline 18 & $22 .^{x h}$ & 68 & $132^{s h}$ & $243^{h 1}$ & $260^{y}$ & $304^{3}$ & 260 & 243 & 132 & 68 & 22 \\
\hline 19 & $25^{22}$ & $76^{c}$ & $172^{s b}$ & $338^{s b}$ & $408^{s b}$ & $504^{3}$ & 504 & 408 & 338 & 172 & 76 \\
\hline 20 & $30 .^{28}$ & $84^{c}$ & $232^{12}$ & $462^{12}$ & $588^{12}$ & $832^{12}$ & $944^{12}$ & 832 & 588 & 462 & 232 \\
\hline 21 & $31 .^{s}$ & $105^{c}$ & $269^{h 3}$ & $570^{s b}$ & $774^{y}$ & $1184^{y}$ & $1454^{y}$ & 1454 & 1184 & 774 & 570 \\
\hline 22 & $37 .^{m}$ & $132^{m}$ & $319^{8}$ & $759^{n 2}$ & $1139^{y}$ & $1792^{y}$ & $2182^{y}$ & $2636^{t 2}$ & 2182 & 1792 & 1139 \\
\hline 23 & $40 .^{14}$ & $147^{\prime 2}$ & $399^{s}$ & $969^{\circ}$ & $1436^{y}$ & $2271^{y}$ & $2970^{y}$ & $3585^{y}$ & 3585 & 2970 & 2271 \\
\hline 24 & $42 .^{s}$ & $168^{s}$ & $532^{s}$ & $1368^{s}$ & $1882^{y d}$ & $3041^{y a}$ & $4200^{y}$ & $5267^{y}$ & $5616^{y}$ & 5267 & 4200 \\
\hline 25 & $500^{s}$ & $2100^{s}$ & $700^{s}$ & $1900^{x}$ & $2590^{y d}$ & $4127^{y a}$ & $6036^{y a}$ & $7960^{y}$ & $9031^{\text {ya }}$ & 9031 & 7960 \\
\hline 26 & $52 .^{c}$ & $260 .^{s}$ & $910^{s}$ & $2600^{5}$ & $3532^{y d}$ & $5703^{y}$ & $8695^{y a}$ & $12037^{y a}$ & $14836^{g s}$ & $15977^{8 s}$ & 14836 \\
\hline 27 & $54 .^{c}$ & 260 & $1170 .^{s}$ & $35100^{*}$ & $4786^{y d}$ & $7727^{y a}$ & $12368^{y a}$ & $18096^{y a}$ & $23879^{g s}$ & $27553^{8 s}$ & 27553 \\
\hline 28 & $63 .^{s s}$ & $272^{y}$ & 1170 & $4680 . s^{s s}$ & $6315^{y d}$ & $10313^{y a}$ & $17447^{y a}$ & $28368^{8.5}$ & $40188^{y^{s}}$ & $49462^{8 s}$ & $52995^{85}$ \\
\hline
\end{tabular}

(or square 2-design) is a 2-design with as many blocks as points.

We have included some codcs that are close to the best presently known when they are easy to construct and the best code is not. These nonrecord codes are indicated by $\ddagger$.

\section{The Tables of $A(n, d, w)$ and $A(n, d)$}

We begin with the main tables, Tables I and II, which give lower bounds on $A(n, d, w)$ and $A(n, d)$. The rest of the paper is devoted to describing the codes in Table I (Table II being largely self-explanatory).
Most of the entries in Table I are new, either because we have improved the lower bound, or because we have found a more symmetric code or more compact definition than was known before. The notes to Table I describe the simplest construction we know for a code with the given parameters. We have usually not attempted to indicate the original discoverer of a code. For as mentioned in Section I our chief concern is to describe thcse codes explicitly. Those interested in the history of these codes may consult the extensive bibliography (see for example [11], [13], [23], [69], [103], [132], [177], [178]) and the Acknowledgment at the end of the paper. Table II contains one new entry, $A(25,10) \geq 151$. 
TABLE I-C

Lowek BUunds on $A(n, 8, w)$

\begin{tabular}{|c|c|c|c|c|c|c|c|c|c|c|}
\hline$n, w$ & 5 & 6 & 7 & 8 & 9 & 10 & 11 & 12 & 13 & 14 \\
\hline 10 & 2. & 2 & 1 & 1 & 1 & 1 & 0 & 0 & 0 & 0 \\
\hline 11 & 2. & 2 & 2 & 1 & 1 & 1 & 1 & 0 & 0 & 0 \\
\hline 12 & $3 .^{j}$ & $4 .^{d}$ & 3 & 3 & 1 & 1 & 1 & 1 & 0 & 0 \\
\hline 13 & 3. & 4. & 4 & 3 & 3 & 1 & 1 & 1 & 1 & 0 \\
\hline 14 & $4 .^{s}$ & 7. ${ }^{d}$ & $8 .^{s}$ & 7 & 4 & 3 & 1 & 1 & 1 & 1 \\
\hline 15 & $6^{a}$ & $10)^{s}$ & $15^{c}$ & 15 & 10 & 6 & 3 & 1 & 1 & 1 \\
\hline 16 & 6. & $16^{9^{2}}$ & $16^{c}$ & $30)^{h m}$ & 16 & 16 & 6 & 4 & 1 & 1 \\
\hline 17 & $7 .^{s}$ & $17^{p c}$ & $24 e^{e c}$ & $34 .^{c}$ & 34 & 24 & 17 & 7 & 4 & 1 \\
\hline 18 & $9 .^{s}$ & $21 .^{93}$ & $33^{s}$ & $46^{s}$ & $48^{s}$ & 46 & 33 & 21 & 9 & 4 \\
\hline 19 & $12^{s}$ & $28 .^{s}$ & $52^{*}$ & $78^{s}$ & $88^{s}$ & 88 & 78 & 52 & 28 & 12 \\
\hline 20 & $16 .^{5}$ & $40 .^{s}$ & $80)^{s}$ & $130)^{5}$ & $160)^{5}$ & $176^{s}$ & 160 & 130 & 80 & 40 \\
\hline 21 & $21 .^{c}$ & $56 . s$ & $120)^{s}$ & $2100^{s}$ & $280)^{s}$ & $336^{s}$ & 336 & 280 & 210 & 120 \\
\hline 22 & 21. & 77:" & $176 .^{s}$ & 330." & 280 & $616^{s}$ & $672^{s}$ & 616 & 280 & 330 \\
\hline 23 & 23 & 77 & $253 . "$ & $506 . "$ & $4000^{r}$ & 616 & $1288^{\circ}$ & 1288 & 616 & 460 \\
\hline 24 & $24^{c}$ & $78^{\times 2}$ & 253 & $759 .^{s s}$ & $640^{\prime 4}$ & $960^{t 4}$ & 1288 & $2576 .^{.4}$ & 1288 & 960 \\
\hline 25 & $30 .^{s}$ & $100)^{s}$ & $254^{x^{2}}$ & 759 & $829^{15}$ & $1248^{y a}$ & $1662^{15}$ & 2576 & 2576 & 1662 \\
\hline 26 & 30. & $130 . s s$ & $2.57^{\prime}$ & $760^{\times 2}$ & $883^{y}$ & $1.519^{15}$ & $1988^{y a}$ & $3070^{y}$ & $3328^{y a}$ & 3070 \\
\hline 27 & 30 & 130 & $278^{y}$ & $766^{x y}$ & $970^{y}$ & $1597^{y}$ & $2295^{y}$ & $3335^{y a}$ & $3923^{y}$ & 3923 \\
\hline 28 & $33 .^{m}$ & 130 & $296^{\prime}$ & $833^{y}$ & $1107^{y}$ & $1806^{y e}$ & $2756^{y / 2}$ & $4114^{y a}$ & $4805^{y a}$ & $5280^{t 6}$ \\
\hline
\end{tabular}

TABLE I-D

LOWER BOUNDS ON $A(n, 10, w)$

\begin{tabular}{|c|c|c|c|c|c|c|c|c|c|}
\hline$n, w$ & 6 & 7 & 8 & 9 & 10 & 11 & 12 & 13 & 14 \\
\hline 12 & 2. & 2 & 1 & 1 & 1 & 1 & 1 & () & 0 \\
\hline 13 & 2. & 2 & 2 & 1 & 1 & 1 & 1 & 1 & 0 \\
\hline 14 & 2. & 2. & 2 & 2 & 1 & 1 & 1 & 1 & 1 \\
\hline 15 & $3 . .^{\prime}$ & $3 . !$ & 3 & 3 & 3 & 1 & 1 & 1 & 1 \\
\hline 16 & 3. & $4 .^{j}$ & $4 !$ & 4 & 3 & 3 & 1 & 1 & 1 \\
\hline 17 & 3. & $5 .^{\prime}$ & $6 !$ & 6 & 5 & 3 & 3 & 1 & 1 \\
\hline 18 & $4 !^{\prime}$ & $6 !$ & $9 .^{42}$ & $10 ?^{s}$ & 9 & 6 & 4 & 3 & 1 \\
\hline 19 & 4. & $8 .^{x}$ & $12^{s b}$ & $19)^{c}$ & 19 & 12 & 8 & 4 & 3 \\
\hline 20 & $5^{s}$ & $10.9^{2}$ & $17^{m}$ & 20 & $38 .^{\mathrm{km}}$ & 20 & 17 & 10 & 5 \\
\hline 21 & 7.4 & $13 .^{x h}$ & 21 & $27^{p c}$ & 38 & 38 & 27 & 21 & 13 \\
\hline 22 & 7. & $16^{p c}$ & $24^{5 i}$ & $35^{p r}$ & $42^{e r}$ & $46^{\prime}$ & 42 & 35 & 24 \\
\hline 23 & $8 .^{x 2}$ & $20)^{y}$ & $33^{p c}$ & $45^{p c}$ & $54^{p c}$ & $63^{p c}$ & 63 & 54 & 45 \\
\hline 24 & $9 .^{\times 2}$ & $24^{\circ}$ & $38^{p c}$ & $56^{c}$ & $72^{c}$ & $90^{c}$ & $96^{c}$ & 90 & 72 \\
\hline 2.5 & $10 .^{s}$ & $28^{e c}$ & $48^{e r}$ & $72^{e c}$ & $100^{\circ}$ & $125^{c}$ & $130^{e c}$ & 130 & 125 \\
\hline 26 & $13 .^{42}$ & 28 & $54^{p c}$ & $84^{p c}$ & $130^{\circ}$ & $168^{p c}$ & $185^{y}$ & $191^{y}$ & 185 \\
\hline 27 & $14 .^{49}$ & $36^{4} 6^{3}$ & $66^{p r .}$ & $111^{c}$ & $159^{\circ}$ & $213^{y a}$ & $257^{y}$ & $283^{y a}$ & 283 \\
\hline 28 & $16 .^{m}$ & $37^{9^{4}}$ & $78^{p c}$ & $132^{p c}$ & $195^{y d}$ & $280^{y a}$ & $356^{y a}$ & $414^{y a}$ & $435^{y d}$ \\
\hline
\end{tabular}

Key to Table I

An entry followed by a period is known to be exact.

$a=$ From a trivial design or its dual (Section III).

$c=$ Cyclic code (Table XI).

$\mathrm{cm}=$ Conference matrix code ((19) of Section III).

$d=$ Doubling ((2) of Section III).

$d 1=2-(25,9,3)$ design (Section III).

$d 2=$ From $(r, \lambda)$-design (Section III).

$e c=$ Extended cyclic (or "cyclic with fixed point") code (Table XII).

$g$ = Group code-orbits under a group with more than one generator (Table XV).

$g f=$ Group code plus extra vectors (Table XV).

$g p=$ Group code followed by polishing-group code need not be subcode (Table XV).

$g s=$ From $S_{2}$-sets (Theorem 16 and Table V).

hm = Hadamard matrix code (Theorem 10).

$h n=$ From Hadamard matrix of order 12 (Section III).

$h 1=$ From Theorem 20 .

$h 2=$ From Theorem 21. h3 = Hämäläinen (see remark following Theorem 21).

$j=$ Juxtaposing ((1) of Section III). Details are left to the reader.

$m=$ Miscellaneous construction (Section XI).

$p 0=$ Partitioning construction with $n_{1}=[n / 2], \epsilon=0$ (Section VI).

$p 1=$ Partitioning construction with $n_{1}=[n / 2], \epsilon=1$ (Section VI).

$p 2=$ Partitioning construction with $n_{1}=[n / 2]-1, \epsilon=0$ (Section VI).

p3 $=$ Partitioning construction with $n_{1}=[n / 2]-1, \epsilon=1$ (Section VI).

$p c=$ Orbits under a single permutation (Table XIV).

$q i=$ Quasi-cyclic code, for $2 \leq i \leq 9$-fixed by a pcrmutation containing $i$ cycles of length $n / i$ (Table XIII).

$s=$ Section of code below or diagonally down to right, obtained from (5) of Section III.

$s b=$ Section of code below, obtained by direct examination of the code (Section III). 
TABLE I-E

LOWer Bounds on $A(n, 12, w)$

\begin{tabular}{|c|c|c|c|c|c|c|c|c|}
\hline$n, w$ & 7 & 8 & 9 & 10 & 11 & 12 & 13 & 14 \\
\hline 14 & 2 & 2 & 1 & 1 & 1 & 1 & 1 & 1 \\
\hline 15 & 2. & 2 & 2 & 1 & 1 & 1 & 1 & 1 \\
\hline 16 & 2. & 2. & 2 & 2 & 1 & 1 & 1 & 1 \\
\hline 17 & 2. & 2. & 2 & 2 & 2 & 1 & 1 & 1 \\
\hline 18 & $3 .^{\prime}$ & $3 .^{d}$ & $4 !$ & 3 & 3 & 3 & 1 & 1 \\
\hline 19 & 3. & 3. & 4. & 4 & 3 & 3 & 3 & 1 \\
\hline 20 & 3. & $5^{d}$ & 5. & $6 .^{a}$ & 5 & 5 & 3 & 3 \\
\hline 21 & 3. & 5. & 7. & $7 . j$ & 7 & 7 & 5 & 3 \\
\hline 22 & $4 .^{j}$ & $6 .^{d}$ & $8^{p c}$ & $11^{d}$ & $12^{s}$ & 11 & 8 & 6 \\
\hline 23 & 4. & 6. & $10 .^{s}$ & $16^{x h}$ & $23 .^{c}$ & 23 & 16 & 10 \\
\hline 24 & 4. & $9 .^{d}$ & $16 .^{s}$ & $24 .^{c}$ & $24^{c}$ & $46 .^{\mathrm{hm}}$ & 24 & 24 \\
\hline 25 & $5^{j}$ & $10 . .^{95}$ & $25 .^{d 1}$ & $28^{p c}$ & $36^{e c}$ & $50^{c m}$ & 50 & 36 \\
\hline 26 & 5. & $13^{d}$ & $26^{c}$ & 3()$^{p c}$ & $39^{a 2}$ & $54^{29}$ & $58^{29}$ & 54 \\
\hline 27 & $6 .^{s}$ & 15.99 & $39 .{ }^{29}$ & $39^{e c}$ & $54^{c}$ & $82^{17}$ & $81^{17}$ & 81 \\
\hline 28 & $8 .^{a}$ & $19 .^{p c}$ & 39 & $48^{8}$ & $63^{p c}$ & $84^{c}$ & $96^{p r}$ & $106^{m}$ \\
\hline
\end{tabular}

TABLE I-F

LOWER BOINDS ON $A(n, 14, w)$

\begin{tabular}{|c|c|c|c|c|c|c|c|}
\hline$n, w$ & 8 & 9 & 10 & 11 & 12 & 13 & 14 \\
\hline 16 & 2. & 2 & 1 & 1 & 1 & 1 & 1 \\
\hline 17 & 2. & 2 & 2 & 1 & 1 & 1 & 1 \\
\hline 18 & 2. & 2. & 2 & 2 & 1 & 1 & 1 \\
\hline 19 & 2. & 2. & 2 & 2 & 2 & 1 & 1 \\
\hline 20 & 2. & 2. & 2. & 2 & 2 & 2 & 1 \\
\hline 21 & $3 !$ & $3 .^{j}$ & $3 .^{j}$ & 3 & 3 & 3 & 3 \\
\hline 22 & 3. & 3. & $4 !$ & $4 !$ & 4 & 3 & 3 \\
\hline 23 & 3. & 3. & 4. & 4. & 4 & 4 & 3 \\
\hline 24 & 3. & 4.' & $5 .^{\prime}$ & $6 . j$ & $6^{j}$ & 6 & 5 \\
\hline 25 & 3. & $5 .^{j}$ & $6 .^{j}$ & 7.. & 8. & 8 & 7 \\
\hline 26 & $4 .^{j}$ & $6 . '$ & $8 \cdot d 2$ & $10 .^{j}$ & $13 .^{9^{2}}$ & $14 .^{s}$ & 13 \\
\hline 27 & 4. & 6. & 9. ${ }^{j}$ & $133^{s}$ & $19^{q 9}$ & $27 .^{s}$ & 27 \\
\hline 28 & 4. & 7. & $11 x^{x}$ & $21^{.94}$ & $28^{c}$ & $28^{c}$ & $54^{\mathrm{hm}}$ \\
\hline
\end{tabular}

$s d=$ Section of code diagonally down to right, obtained by direct examination of the code (Section III).

$s f=$ Section of code below or diagonally down to right, followed by addition of extra vectors (Scction XI).

$s p=$ Section of code below or diagonally down to right, followed by polishing (Section XI).

$s s=$ From Steiner systems $S(2,3,7), \quad S(3,4,8)$, $S(5,6,12), \quad S(3,4,14), \quad S(3,4,16), \quad S(5,6,24)$, $S(3,4,26), \quad S(3,4,28)$ for $d=4 ; \quad S(3,5,17)$, $S(2,4,28), S(5,7,28)$ for $d=6 ; S(5,8,24), S(3,6,26)$ for $d=8$ (Table IV)

$t 1=$ From translate of Nordstrom-Robinson code (Section IX).

t2 = Adding tails to translates of Nordstrom-Robinson code (Section IX).

$t 4=$ From translate of Golay code (Section IX).

t5 = Adding tails to translates of Golay code (Section IX).

$t 6=$ From translate of Karlin's [27, 14, 7] code (Section IX).

$t 7=$ From translate of $[26,7,11]$ code (Section IX).

$x=$ Lexicographic code (Section VIII).

$x h=$ Lexicode with sccd (Table VIII).

$x y=$ Lexicode with seed (Table XVI).

$x 2=$ Complement of lexicode with sum constraint (Table VII).

$y=$ No known structure (Table XVI).
TABLE I-G

VALLES OF $A(n, 16, w)$

\begin{tabular}{c|cccccc}
\hline \hline$n, w$ & 9 & 10 & 11 & 12 & 13 & 14 \\
\hline 18 & 2. & 2 & 1 & 1 & 1 & 1 \\
19 & 2. & 2 & 2 & 1 & 1 & 1 \\
20 & 2. & 2. & 2 & 2 & 1 & 1 \\
21 & 2. & 2. & 2 & 2 & 2 & 1 \\
22 & 2. & 2. & 2. & 2 & 2 & 2 \\
23 & 2. & 2. & 2. & 2 & 2 & 2 \\
24 & $3 . j$ & $3 .{ }^{d}$ & $3 . j$ & $4 . .^{d}$ & 3 & 3 \\
25 & 3. & 3. & 3. & 4. & 4 & 3 \\
26 & 3. & 3. & $4 !$ & 4. & 4. & 4 \\
27 & 3. & 3. & $5 . j$ & $5 !^{j}$ & $6 .^{j}$ & 6 \\
28 & 3. & $4 .{ }^{d}$ & 5. & $7 .^{d}$ & $7 .^{j}$ & $8 .{ }^{d}$ \\
\hline
\end{tabular}

TABLE I-H

V ALUES OF $A(n, 18, w)$

\begin{tabular}{c|ccccc}
\hline \hline$n, w$ & 10 & 11 & 12 & 13 & 14 \\
\hline 20 & 2. & 2 & 1 & 1 & 1 \\
21 & 2. & 2 & 2 & 1 & 1 \\
22 & 2. & 2. & 2 & 2 & 1 \\
23 & 2. & 2. & 2 & 2 & 2 \\
24 & 2. & 2. & 2. & 2 & 2 \\
25 & 2. & 2. & 2. & 2 & 2 \\
26 & 2. & 2. & 2. & 2. & 2 \\
27 & 3. & 3. & 3. & 3. & 3 \\
28 & 3. & 3. & 3. & $4 !$ & $4 !$
\end{tabular}

$y a=$ Obtaincd by extending the code above it in the table; no other known structurc.

$y d=$ Obtained by extending the code diagonally above it to left; no other known structure.

$z 2=[170]$.

$z 3=$ Theorem 4 .

$z 4=$ Theorem 6 .

$z 5=$ Theorem 5 .

$28=$ [22].

$z 9=[95]($ see Table XVI)

Key to Table II

Unmarked entries are either trivial or are obtained by

shortening the code below.

An entry followed by a period is known to be exact.

1 = Extended Hamming code ([132], p. 23).

2 = Conferencc matrix code ([132], p. 585, [165]).

3 = Best ([12], [45], p. 140).

4 = From $S(5,6,12), 6$ disjoint words of weight 2 and complements ([45], p. 139, [132], p. 585).

5 = Romanov [155]-see Section VI.

$6=$ From Hamming code over GF(5) [79].

$7=$ From the $u \mid u+v$ construction ([132], p. 76, [166]).

8 = Hadamard matrix codc ([132], p. 49).

$8 a=$ "Nadler" code ([13], [129], [132], pp. 75, 79).

$9=$ Nordstrom-Robinson code (Section IX, [5], [132], p. 73).

$10=$ Nonlinear code from Construction X ([132], p. 583, [164], p. 505).

11 = From Construction X4 ([132], p. 585, Example 7, [164], p. 507).

12 = Wagner [179]. 
TABLE II

LOWER BOUNDS ON $\boldsymbol{A}(n, d)$

\begin{tabular}{|c|c|c|c|c|c|c|c|c|c|}
\hline$n, d$ & 4 & 6 & 8 & 10 & 12 & 14 & 16 & 18 & 20 \\
\hline 5 & 2. & 1. & 1. & 1. & 1. & 1. & 1. & 1. & 1. \\
\hline 6 & 4. & 2. & 1. & 1. & 1. & 1. & 1. & 1. & 1. \\
\hline 7 & 8. & 2. & 1. & 1. & 1. & 1. & 1. & 1. & 1. \\
\hline 8 & $16 .^{1}$ & 2. & 2. & 1. & 1. & 1. & 1. & 1. & 1. \\
\hline 9 & $20 .^{2}$ & 4. & 2. & 1. & 1. & 1. & 1. & 1. & 1. \\
\hline 10 & $40 .^{3}$ & 6. & 2. & 2. & 1. & 1. & 1. & 1. & 1. \\
\hline 11 & 72 & 12. & 2. & 2. & 1. & 1. & 1. & 1. & 1. \\
\hline 12 & $144^{4}$ & $24 .^{8}$ & 4. & 2. & 2. & 1. & 1. & 1. & 1. \\
\hline 13 & 256. & $32 .^{8 a}$ & 4. & 2. & 2. & 1. & 1. & 1. & 1. \\
\hline 14 & 512. & 64. & 8. & 2. & 2. & 2. & 1. & 1. & 1. \\
\hline 15 & 1024 & 128. & 16. & 4. & 2. & 2. & 1. & 1. & 1. \\
\hline 16 & $2048 .{ }^{1}$ & $256^{9}$ & $32 .^{14}$ & 4. & 2. & 2. & 2. & 1 & 1. \\
\hline 17 & $2720^{5}$ & 256 & $36^{2}$ & 6.17 & 2. & 2. & 2. & 1. & 1. \\
\hline 18 & 5248 & 512 & 64 & I). & 4. & 2. & 2. & 2. & 1. \\
\hline 19 & $10496^{6}$ & 1024 & 128 & 20. & 4. & 2. & 2. & 2. & 1. \\
\hline 20 & $2048\left(0^{7}\right.$ & $2048^{10}$ & 256 & $40^{8}$ & $6^{17}$ & 2. & 2. & 2. & 2. \\
\hline 21 & 36864 & $2560^{11}$ & 512. & $42^{18}$ & 8.17 & 4. & 2. & 2. & 2. \\
\hline 22 & 73728 & $4(096$ & 1024 & $48^{17}$ & 12. & 4. & 2. & 2. & 2. \\
\hline 23 & 147456 & 8192 & 2048. & $68^{19}$ & 24. & 4. & 2. & 2. & 2. \\
\hline 24 & $294912^{7}$ & $16384^{12}$ & $4096 . .^{15}$ & $128^{20}$ & $48^{8}$ & $6 .^{17}$ & 4. & 2. & 2. \\
\hline 25 & 524288 & 16384 & 4096 & $151^{21}$ & $52^{2}$ & $8 .^{17}$ & 4. & 2. & 2. \\
\hline 26 & 1048576 & 32768 & 4096 & 256 & 64 & 14. & 4. & 2. & 2. \\
\hline 27 & 2097152 & 65536 & 8192 & 512 & $128^{23}$ & 28 & $6 .^{17}$ & 4. & 2. \\
\hline 28 & $4194304^{1}$ & $131072^{13}$ & $16384^{16}$ & $1024^{22}$ & 128 & $56 .^{8}$ & 8.17 & 4. & 2. \\
\hline
\end{tabular}

13 = Shortened nonprimitive $\mathrm{BCH}$ code of length 32 ([132], p. 586).

14 = Reed-Muller code ([132], Chap. 13).

15 = Golay code ([45], Chaps. 3, 11, [132], Chap. 20).

16 = Sclf-dual double circulant code ([45], p. 189, [132], p. 509, [148]) - see (53).

17 = From Hadamard matrices using Levenshtein's construction ([122], [132], p. 50).

$18=$ Extended quasi-cyclic code [104].

$19=$ Extended cyclic code [105] (see Table XI).

20 = Hashim-Pozdniakov linear codc [88].

21 = Cyclic code (see Table XI).

22 = Piret [147].

23 = Linear code ((51) of Section IX, [87], [89], [132], p. 593).

We see from Table I that the exact value of $A(n, d, w)$ is now known for all lengths $n \leq 11$ (the first undetermined value being $80 \leq A(12,4,5) \leq 84)$. Similarly, from Tablc II, $A(n, d)$ (for $d$ even) is known exactly for $n \leq 10$, the first undetermined value being $72 \leq A(11,4) \leq 79$ (the upper bound is from [12]).

Of course there is no theoretical difficulty in computing $A(n, d)$ or $A(n, d, w)$. One "simply" forms the graph with $2^{n}$ or $\left(\begin{array}{l}n \\ w\end{array}\right)$ vertices, corresponding to all possible codewords, joins two vertices by an edge if their Hamming distance is at least $d$, and finds the largest clique. Although several new algorithms have recently been proposed for clique-finding (see [6], [7], [70], [71], [140]), the unsolved problems in Tables I and II appear to be beyond their range. Similarly, finding the largest code invariant under a given permutation group (see Section $\mathrm{X}$ ) requircs finding the largest clique in a graph with weights attached to the vertices.

\section{Johnson and Relateid Buuniss}

S. M. Johnson [99]-[102] obtained a number of upper bounds on $A(n, d, w)$, given in Theorems 2,8 and (7), (16), (18). The cascs when equality holds in these bounds (see Conjecture 3 and Theorems 4-7, 9-13) are of particular interest, because of their connection with Steiner systems, block designs and Hadamard matrices. We also mention some related lower bounds obtained from conference matrices.

\section{Theorem 1 (Trivial values):}

a) If $d$ is odd, $A(n, d, w)=A(n, d+1, w)$.

b) $A(n, d, w)=A(n, d, n-w)$.

c) $A(n, d, w)=1$ if $2 w<d$.

d) If $d=2 w$ then $A(n, d, w)=\lfloor n / w\rfloor$

e) $A(n, 2, w)=\left(\begin{array}{l}n \\ w\end{array}\right)$.

Remark: Lct $N(a, d, w)$ denote the inverse of $A(n, d, w)$, that is, the smallest $n$ for which one can find $a$ words of weight $w$ at mutual distance $d$, where $d$ is even. Then one can show that

$$
\begin{aligned}
& N(0, d, w)=0, \\
& N(1, d, w)=w,
\end{aligned}
$$

and if $w \geq d / 2$ then

$$
\begin{aligned}
& N(2, d, w)=w+d / 2, \\
& N(3, d, w)=\max \{3 d / 2, w+d / 2\}, \\
& N(4, d, w)=\max \{3 d-2 w,[d+2 w / 3\rceil, w+d / 2\}
\end{aligned}
$$

(if $w<d / 2$ these quantities do not exist).

By juxtaposing two codes-placing them side by side - we obtain

$$
\begin{aligned}
A\left(n_{1}+n_{2}, d_{1}\right. & \left.+d_{2}, w_{1}+w_{2}\right) \\
& \geq \min \left\{A\left(n_{1}, d_{1}, w_{1}\right), A\left(n_{2}, d_{2}, w_{2}\right)\right\} .
\end{aligned}
$$


In particular we have the doubling construction:

$$
A(2 n, 2 d, 2 w) \geq A(n, d, w) .
$$

Lower bounds in Table I obtained from (1), (2) are indicated by " $j$ " and " $d$ " respectively.

Theorem 2 (.Johnson; [132], p. 527, [176], p. 98):

$$
\begin{aligned}
& A(n, d, w) \leq\left\lfloor\frac{n}{w} A(n-1, d, w-1)\right\rfloor, \\
& A(n, d, w) \leq\left\lfloor\frac{n}{n-w} A(n-1, d, w)\right\rfloor .
\end{aligned}
$$

In particular

$$
A(n, d, w) \leq A(n-1, d, w-1)+A(n-1, d, w) .
$$

We also use the contrapositives to (3a), (3b): if there exists a code showing that $A(n, d, w) \geq M$, then

$$
\begin{aligned}
A(n-1, d, w-1) \geq & \left\lceil\frac{w M}{n}\right\rceil, \\
& A(n-1, d, w) \geq\left\lceil\frac{n-w}{n} M\right\rceil .
\end{aligned}
$$

Lower bounds obtained from (5) are indicated by "s" (for "Section") in Tablc I. In some cases these inequalities can be improved by examining the code and finding a column with an exceptionally large number of 0's or 1's (and deleting that column). Bounds obtained in this way are indicated by "sb" or " $s d$ " in Table $\mathrm{I}$.

The first Johnson bound $J_{1}(n, d, w)$ is defined to be the smallest upper bound on $A(n, d, w)$ that is obtained by repeatedly applying (3a) and (3b) until Theorem 1 can be used. For example

$$
J_{1}(n, 4,3)= \begin{cases}\left\lfloor\frac{n}{3}\left\lfloor\frac{n-1}{2}\right\rfloor\right], & \text { if } n \neq 5(\bmod 6) \\ \left\lfloor\frac{n}{3}\left\lfloor\frac{n-1}{2}\right\rfloor\right\rfloor-1, & \text { if } n \equiv 5(\bmod 6) .\end{cases}
$$

Clearly

$$
A(n, d, w) \leq J_{1}(n, d, w),
$$

and thcre are reasons for believing that this bound may be tight when $n$ is sufficiently large.

Conjecture 3: For $d, w$ fixed,

$$
A(n, d, w)=J_{1}(n, d, w)
$$

for all sufficiently large $n$. If true this would be a remarkably strong result (it would imply for example by Theorem 7 that Stciner systems $S(t, k, v)$ cxist for all $t$ and $k$ provided $v$ is sufficiently large and satisfies the obvious congruences). Rödl [154] has shown that $A(n, d, w) /$ $J(n, d, w)$ approaches 1 as $n \rightarrow \infty$ for any fixed $d$ and $w$. An asymptotic result for certain values of $w$ and $d$ growing with $n$ was given in [186]. The conjecture is known to be true in a few cases, as we shall now see.

Theorem 4 (Kirkman [109], Schönheim [157]; see also [77], p. 237, [106], [153], (169)):

$$
A(n, 4,3)=J_{1}(n, 4,3)(\text { see }(6)) \text { holds for all } n \text {. }
$$

Theorem 5 (Brouwer [21], Hanani [81], Kalbfleisch and Stanton [106], Schönheim [157]): For $n \neq 5(\bmod 6)$,

$$
A(n, 4,4)=J_{1}(n, 4,4) \text {, }
$$

the values of this function being

$$
\begin{array}{ll}
\frac{n(n-1)(n-2)}{24}, & \text { if } n \equiv 2 \operatorname{or} 4(\bmod 6) \\
\frac{n(n-1)(n-3)}{24}, & \text { if } n \equiv 1 \operatorname{or} 3(\bmod 6) \\
\frac{n\left(n^{2}-3 n-6\right)}{24}, & \text { if } n \equiv 0(\bmod 6) .
\end{array}
$$

For $n \equiv 5(\bmod 6)$ we have $A(5,4,4)=1, A(11,4,4)=35$ ([12], [13], [45, p. 141]), but for larger $n$ the values are unknown. See also [86].

Theorem 6 (Brouwer [22], [29]):

$$
A(n, 6,4)=J_{1}(n, 6,4)
$$

holds for all $n$ except $8,9,10,11,17,19$ (for these values see Table I-B).

One special case of equality in the first Johnson bound is particularly important.

Theorem 7 (Schönheim [157]; see also [132], p. 528, [160], [176], p. 100):

$$
A(n, 2 \delta, w)=\frac{n(n-1) \cdots(n-w+\delta)}{w(w-1) \cdots \delta}
$$

if and only if a Steiner system $S(w-\delta+1, w, n)$ exists.

In this case equality also holds in (7); the codewords are the blocks of the corresponding Steiner system. The codes obtained in this way are discussed in Section IV.

Thcorem 7 enables us to write down immediately the parameters of the codes corresponding to the blocks of a Steiner system. The blocks of an arbitrary $2-(v, k, \lambda)$ design form a code showing that $A(v, d, k) \geq b$, but in general $d$ is not determined by the other parameters. However, the blocks of the dual or transpose design (obtained by interchanging points and blocks) form a code showing that

$$
A(b, 2(r-\lambda), r) \geq v .
$$

If the design is symmetric these two codes have the same parameters. Theorem 9 describes a case where equality holds both in (14) and in another of Johnson's bounds.

Theorem 8 (Johnson [99]; [132, p. 526]): Let $A(n, 2 \delta, w)$ $=M$, and write $w M=a n+b, 0 \leq b<n$. Then (by considering the sum of the inner products of all pairs of codewords)

$$
(n-b) a(a-1)+b a(a+1) \leq(w-\delta) M(M-1) .
$$

Theorem 8 rules out certain combinations of $n, d, w, M$ (see [132, p. 526] for an example). The second Johnson bound $J_{2}(n, d, w)$ is defined to be the largest value of $M$ permitted by Theorem 8 (possibly infinity). Clearly

$$
A(n, d, w) \leq J_{2}(n, d, w) .
$$

The proof of Theorem 8 shows that if equality holds in (15) then every pair of distinct codewords has inner prod- 
uct $w-\delta$, and also that the $M \times n$ array formed by the codewords contains $n-b$ columns of weight $a$ and $b$ columns of weight $a+1$. This is the dual of an $(r, \lambda)$ design with $r=w, \lambda=w-\delta$ in the notation of Section I. We mention three examples of such designs.

1) A trivial example occurs when

$$
n=\left(\begin{array}{c}
k \\
a
\end{array}\right), M=k, \quad b=0, \quad w=\left(\begin{array}{c}
k-1 \\
a-1
\end{array}\right),
$$

and the columns of the array consist of all possible binary vectors of weight $a$. Such codes are denoted by " $a$ " in Table I.

2) In the special case $a=2$ (when $w M<3 n$ ), equality holds in (15) if and only if there is a 1-design consisting of $b$ triples from $M$ points, such that no pair is covered more than $w-\delta$ times. For example $A(26,14,9)=6$ can be obtained in this way; we omit the details.

3) To obtain $A(26,14,10)=8$, by the previous remarks we must find 24 triples and 2 quadruples from an 8 -set. If the 8 -sct is $\{a, b, c, d, A, B, C, D\}$ we may use the triples $\{i, j, K\}$ and $\{i, J, K\}$ and the quadruples $\{a, b, c, d\}$, $\{A, B, C, D\}$.

We shall discuss equality in (16) at the end of this section. We next discuss a weaker version of Theorem 8 , also due to Johnson (obtained by ignoring the condition that certain variables must be integers), which states that

$$
A(n, 2 \delta, w) \leq \frac{\delta n}{w^{2}-w n+\delta n},
$$

provided the denominator is positive ([99]; [132], p. 525, [176], p. 97).

Theorem 9 (Semakou and Zinoviev [160]; [176], p. 99): Equality holds in (18) if and only if there exists a 2-design with parameters $h=n, r=w, \lambda=w-\delta, l=\delta n /\left(w^{2}-\right.$ $w n+\hat{\delta} n), k=\delta w /\left(w^{2}-w n+\delta n\right)$.

In Theorem 9 the codewords are the blocks of the dual design; equality then holds in (14). There are lists of small block designs in [77], [133]. The entry $A(25,12,9)=25$ in Table I for example is obtained from Theorem 9 using a symmetric $2-(25,9,3)$ design. There are in fact 78 inequivalent designs with these parameters [58], [133], and so exactly 78 inequivalent codes showing $A(25,12,9)=25$. One example is given in Table XIV.

Hadamard matrices also determine some values of $A(n, d, w)$

Theorem 10 ([160)]; see also [13], Theorem 15, [132], p. 528): A Hadamard matrix $H_{n}$ of order $n \geq 1$ exists if and only if

$$
A\left(n, \frac{n}{2}, \frac{n}{2}\right)=2 n-2 .
$$

The code is constructed from the rows of $H_{n}$ and $-H_{n}$ by making the entries of the first row of $H_{n}$ equal to +1 , then changing +1 to 0 and -1 to 1 in every row. The optimality of these codes follows by applying (3) once and then (18).
A modification of this construction shows that $A(14,6,7) \geq 42$. Takc a Hadamard matrix of order 12, with first row and column containing +1 's, replace +1 's by 0 's and -1 's by 1's, omit the first (zero) row, and label the remaining rows $\rho_{0}, \cdots, \rho_{10}$. Then the code

$$
\begin{array}{cccc}
\frac{\rho_{0}}{\rho_{0}} & 0 & 01 & \\
\rho_{i} & 10 & (1 \leq i \leq 10) \\
\overline{\rho_{i}} & 10 & (1 \leq i \leq 10) \\
\frac{\rho_{0}+\rho_{i}}{0} & 01 & (1 \leq i \leq 10) \\
\overline{\rho_{0}+\rho_{i}} & 01 & (1 \leq i \leq 10)
\end{array}
$$

shows that $A(14,6,7) \geq 42$. This idea appears to succeed only for a Hadamard matrix of order $122^{2}$

Similarly if a conference matrix ([132], p. 55, [165]) of order $n \equiv 2(\bmod 4)$ exists then

$$
A\left(n-1, \frac{n-2}{2}, \frac{n-2}{2}\right) \geq 2 n-2 .
$$

Equality holds in (19) for $n=6,10,14,18$; we would like to know if it holds in general.

Honkala et al. [95] show that if a conference matrix of order $n$ exists then

$$
A(2 n, n, n-1) \geq 2 n,
$$

if $n$ is a multiple of 4 then

$$
A(2 n+1, n, n-1) \geq 2 n+A\left(n, \frac{n}{2}, \frac{n-2}{2}\right),
$$

and if $n$ is a multiple of 4 and a conference matrix of order either $n / 2$ or $n+2$ cxists then

$$
A(2 n+1, n, n-1) \geq 3 n \text {. }
$$

For example (22) gives an alternative proof that $A(25,12,11) \geq 36$ (cf. Table IV).

Finally we return to (16). In 1985 Honkala [92] obtained a considerable generalization of Theorem 10 by showing that equality holds in (16) over a wide range of values. The proof is constructive, by juxtaposing (see (1)) appropriate combinations of Hadamard matrices of orders up to $n$.

Theorem 11 (Honkala [92]): Provided Hadamard matrices of all orders $4 k \leq n$ exist,

$$
A(n, d, w)=J_{2}(n, d, w)
$$

holds whenever

$$
n-d \leq w \leq d .
$$

In particular, if (23) holds and $n<2 d$, then

$$
A(n, d, w)= \begin{cases}2 u, & \text { if } i-v \leq 2 j \leq i+v, \\ 2 u-1, & \text { otherwise }\end{cases}
$$

where $i=2 d-n, j=d-w$ and $d=u i+v$ with $0 \leq v<i$.

\footnotetext{
${ }^{2} \mathrm{~A}$ fact which ultimately depends on the existence of the outer automorphism of the symmetric group $S_{6}$.
} 
TABLE III

Sources for Proof that Ceriain Entries in Tabif. I Marked with Period are Exact*

\begin{tabular}{|c|c|}
\hline Value & Reference \\
\hline$A(12,6,5)=1,2$ & {$[132]$, p. 530.} \\
\hline$A(13,6,5)=18$ & [132], p. 531. \\
\hline$A(14,6,7)=42$ & Exha ustive search (see Section XII). \\
\hline$A(17,6,4)=20$ & {$[20]$.} \\
\hline$A(19,6,4)=25$ & {$[170]$.} \\
\hline$A(16,8,7)=16$ & Exhaustive search (see Section XII). \\
\hline$A(17.8,7)=24$ & Exhaustive search (see Section XII). \\
\hline$A(17,8,8)=34$ & [149]. \\
\hline$A(22.8,5)=21$ & $\begin{array}{l}\text { By the Bose-Conner theorem }([18],[96]) \text { a square } \\
\text { divisible design } G D(5,1,2 ; 11 \times 2) \\
\text { does not exist. }\end{array}$ \\
\hline$A(24,8,12)=2576$ & Linear programming [13]. \\
\hline$A(26,8,5)=30$ & {$[25]$. } \\
\hline$A(20,10,8)=17$ & Exhaustive search (see Section XII). \\
\hline$A(21,10,7)=13$ & $\begin{array}{l}\text { From The orem } 8 \text {, if } A(21,10,7) \geq 14 \text { then in fact } \\
A(21,10,7)-15 ; \text { but }(\text { see }[77]) \\
\text { no } 2-(15,5,2) \text { design exists. }\end{array}$ \\
\hline$A(22,10,7)=16$ & Exhaustive search (see Scction XII). \\
\hline$A(23,10,7)=21$ & Exhaustive search (scc Section XII). \\
\hline$A(28,12,8)=19$ & $\begin{array}{l}\text { From Theorem } 8 \text {, if } A(28,12,8) \geq 20 \text { then in fact } \\
A(28,12,8)=21 \text {; hut }(\text { see }[77]) \\
\text { no } 2-(21,6,2) \text { design exists. }\end{array}$ \\
\hline
\end{tabular}

*Exactness of entries not listed here follows from the Johnson bounds (3), (7), (16)

The examplc $A(26,14,10)=8$ previously mentioned shows that equality may also hold in (16) outside the "Honkala region" (23).

Most proofs that the entries in Table I followed by a period are exact can be obtained from the Johnson bounds ((3), (7), (16)). Sources for proofs that the other entrics are cxact are given in Table III.

\section{Steiner Systems}

In this section we give a highly compressed survey of Steiner systems (defined in Section I). Because some constructions are available only in obscure sources, and because in Section VI wc shall require not just a single Steiner system but as many disjoint ones as possiblc Tablc IV contains a number of explicit constructions for small Steiner systems.

As we saw in the previous section (Theorem 7), if a Steiner system $S(t, k, v)$ exists, it contains $b=\left(\begin{array}{l}c \\ 1\end{array}\right) /\left(\begin{array}{l}k \\ t\end{array}\right)$ blocks, and then $A(v, 2 k-2 t+2, k)=b$. If $S(t, k, v)$ exists then so does the contracted (or derived) design $S(t-1, k-1, v-1)$, formed from all the blocks containing (say) the last point.

Steiner triple systems $S(2,3, v)$ exist if and only if $v \equiv 1$ or 3 (mod 6) (Kirkman [109]; see Theorem 4). Steiner quadruple systems $S(3,4, v)$ exist if and only if $v \equiv 2$ or 4 (mod 6) (Hanani [81]; see Theorem 5).

Theorem 12 (Hanani [82], [83]): $S(2,4, v)$ exists if and only if $v \geq 4$ and $v \equiv 1$ or $4(\bmod 12)$; in these cases

$$
A(v, 6,4)=v(v-1) / 12
$$

$S(2,5, \iota)$ exists if and only if $v \geq 5$ and $\iota \equiv 1$ or $5(\bmod 20)$; in these cases

$$
A(\iota, 8,5)=\imath \cdot(\iota-1) / 20 .
$$

Theorem 13 (See [53], [52], Chap. 6; [84]): For any prime power $q$ and any nonnegative integer $d$ there exist Stciner systems $S\left(2, q, q^{d}\right)$ (from the lines in the affine geometry $A G(d, q)), S\left(2, q+1, q^{d}+q^{d-1}+\cdots+q+1\right)$ (from the lines in the projective geometry $P G(d, q)$ ), $S\left(2, q+1, q^{3}+1\right)$ (from a unital in $P G\left(2, q^{2}\right)$ ), $S\left(2,2^{a}, 2^{a+b}+2^{a}-2^{b}\right)$ for $a \leq b$ (from a complete $2^{a}$-arc in $P G\left(2,2^{b}\right)$ ), $S\left(3, q+1, q^{2}+1\right)$ (from an clliptic quadric in $P G(3, q)$ ), or more generally $S\left(3, q+1, q^{d}+1\right)$ (from subficld sublines of a projective line). Thus

$$
\begin{gathered}
A\left(q^{d}, 2 q-2, q\right)=\frac{q^{d-1}\left(q^{d}-1\right)}{q-1}, \\
A\left(q^{d}+q^{d-1}+\cdots+q+1,2 q, q+1\right) \\
=\frac{\left(q^{d+1}-1\right)\left(q^{d}-1\right)}{\left(q^{2}-1\right)(q-1)}, \\
A\left(q^{3}+1,2 q, q+1\right)=q^{2}\left(q^{2}-q+1\right), \\
=\left(2^{a+h}+2^{a}-2^{b}, 2^{a+1}-2,2^{a}\right)\left(2^{b}-2^{b-a}+1\right), \\
A\left(q^{d}+1,2 q-2, q+1\right)=\frac{q^{d-1}\left(q^{2 d}-1\right)}{q^{2}-1} .
\end{gathered}
$$

The only Steiner systems presently known with $t \geq 4$ are $S(5,6,12), S(5,8,24)$ (Mathieu, Witt [182], [183], [45]), $S(5,6,24), \quad S(5,7,28), \quad S(5,6,48), \quad S(5,6,84)$ (Denniston [56]), and $S(5,6,72)$ (Mills [138]); and their contractions with $t=4$. All these 5-designs are invariant under the group $\operatorname{PSL}(2, v-1)$ for the appropriate value of $\iota$.

Tablc IV lists the known Steiner systems (or $t-(v, k, 1)$ designs) with $v \leq 28$ and $2 \leq t<k ; b$ denotes the number of blocks. For each design we give either a construction or a reference, as well as information about the number of inequivalent designs (up to permutation of coordinates) and the number of disjoint designs. Some designs which are contractions of others have been omitted.

The Steiner triple systems on at most 21 points are treated in great detail in [156]. For further information about Steiner systems see [1], [3], [45], [52], [61], [62], [84], [96], [117], [118], [121], [127], [128], [139], [152], [168], [182], [183].

\section{Graham-Sloane Type: Boundes}

We next discuss lower bounds on $A(n, d, w)$, roughly in order of increasing complexity. The bounds described in this section require very little computation (and correspondingly produce the weakest results). For $d \geq 6$ we give several different bounds, all roughly comparable (although only codes from Theorem 16 are necded for the present version of Table I). 
TABLE IV

STEINER SYSTEMS

$S(2,3,7)=P G(2,2) ; b=7$. Cyclic: $\{(1011000)\}$; unique. Exactly two disjoint designs exist, the other being $\{(1101000)\}$.

$S(3,4,8)=A G(3.2) ; b=14$. Extended cyclic: $\{(1011000) 1,(0100111) 0\}$; unique. Exactly two disjoint designs exist, the other being $\{(1101000) 1$, $(0010111) 0\}$ [32].

$S(2,3,9)=A G(2,3) ; b=12$. It is also the unital in $P G(2,4)$; unique. In each of the following matrices, the 12 triples formed by the rows, columns and six generalized diagonals form an $S(2,3,9)$. The 7 matrices together yield 7 disjoint designs, partitioning the set of all $\left(\begin{array}{l}9 \\ 3\end{array}\right)$ triples [109].

$\begin{array}{lllllll}124 & 128 & 125 & 129 & 123 & 126 & 127 \\ 378 & 943 & 983 & 743 & 469 & 357 & 346 . \\ 956 & 765 & 476 & 586 & 785 & 489 & 598\end{array}$

There are exactly two inequivalent partitions [9].

$S(3,4,10) ; b=30$. Cyclic; unique. Exactly five disjoint designs exist, in a unique way [115]. For example, we may take the five designs defined by the columns of the following array. The six hexadecimal vectors in a column together with their images under the permutation $(1,2,3,4,5)(6,7,8,9,10)$ form an $S(3,4,10)$; the five columns give five disjoint designs (cf. Section $\mathrm{X}$ ).

$\begin{array}{rrrrr}27 & 2 B & 2 D & 2 E & 36 \\ 3 A & 3 C & 33 & 35 & 39 \\ 69 & 65 & 74 & 72 & 6 A \\ B 1 & A 6 & B 8 & A 3 & A C \\ E 4 & F 0 & E 2 & E 8 & E 1 \\ 170 & 162 & 168 & 161 & 164\end{array}$

$S(4.5,11) ; b=66$. Unique. Exactly two disjoint designs exist, in a unique way [115]. For example. the designs $\left\{7 A 0^{55}, 712^{11}\right\}$ and $\left\{782^{55}, 748^{11}\right\}$ defined by the group of order 55 generated by $(1,2,3, \cdots, 11)$ and $(2,5,6,10,4)(3,9,11,8,7)$ (cf. Table XV).

$s(5,6,12) ; b=132$. Unique. Exactly two disjoint designs exist, in unique way [115]. An $S(5,6,12)$ may be oblained from an $S(4,5,11)$ by appending 1 and adjoining the complements of all blocks. Two disjoint $S(5,6,12)$ 's arise from the two previous disjoint $S(5,6,11)$ 's. Alternatively, two disjoint designs arise from the supports of the codewords of weight 6 in the two extended ternary quadratic residue codes of length 12 whose zeros are the residues and nonresidues modulo 11 respectively [2], [3].

The prototype of these bounds is the following.

Theorem 14 ([72]):

$$
A(n, 4, w) \geq \frac{1}{n}\left(\begin{array}{l}
n \\
w
\end{array}\right) .
$$

This is established by defining a map $f$ from vectors $a=\left(a_{0}, a_{1}, \cdots, a_{n-1}\right) \in \mathbb{F}_{2}^{n}$ to $\mathbb{Z}_{n}$ by

$$
f(a)=\sum_{i=0}^{n-1} i a_{i}(\bmod n) .
$$

Then it is easy to see that the sets $C_{0}^{(w)}, C_{1}^{(w)}, \cdots, C_{n-1}^{(w)}$, where

$$
C_{k}^{(w)}=\left\{a \in \mathbb{F}_{2}^{n}: f(a)=k, \quad w t(a)=w\right\},
$$

form a partition of the set of all $\left(\begin{array}{l}n \\ w\end{array}\right)$ binary vectors of weight $w$ into $n$ disjoint codcs each with Hamming distance 4 . Since one of the $C_{k}^{(w)}$ must contain at least as many words as the average, (31) follows.
$S(2,3,13) ; b=26$. There are exactly two inequivalent designs ([77] 237), one of which is cyclic (Table XI). Denniston [54] (see also [116]) partitioned the set of all $\left(\begin{array}{c}13 \\ 3\end{array}\right)$ triples into 11 disjoint designs.

$S(2,4,13)=P G(2,3) ; b=13$. Cyclic; unique. Chouinard [36] partitioned the set of all $\left(\begin{array}{c}13 \\ 4\end{array}\right) 4$-sets into 55 disjoint designs.

$S(3,4,14) ; b=91$. There are exactly four inequivalent designs, one of which is given in Table XV [137]. A set of four disjoint designs (not hitherto known to exist) is obtained by applying the permutations $(1,2,4,7,10,13,12,14,3,6,8,5,9,11),(1,4,8,10,2,11,3,5,7,6,12,13,9)$ and $(1,6,9,14,8,11,12,10,5,4,3)(2,7,13)$ to the design in Table XV. See also [116].

$S(2,3,15) ; b=35$. There are exactly 80 inequivalent designs [38], [78], one of which is $P G(3,2)$. Denniston [54], [55] partitioned the set of all $\left(\begin{array}{c}15 \\ 3\end{array}\right)$ triples into 13 disjoint designs.

$S(2,4,16)=A G(2,4) ; b=20$. The weight 4 words in a translate of the Nordstrom-Robinson code (see Table X); unique. There exists an $S(3,4,16)$ that is tiled hy 7 copies of $S(2.4,16)$

$S(3,4,16) ; b=140$. For example, the planes in $\mathrm{AG}(4,2)$, the weight 4 words in the $[16,11,4]$ Hamming code. Therc are at least 31301 inequivalent designs [126], [127]. At least 8 pairwise disjoint designs exist (see [112], [124], [125] and the Appendix).

$S(3,5,17) ; b=68$. Unique (see Theorem 13 and Table XV). At least two disjoint designs exist [136], [185]

$S(3,4,20) ; b=285$. From the partitioning construction (Section VI). There are at least $10^{17}$ inequivalent designs [126], and exactly 29 cyclic designs [142]. At least 15 disjoint designs exist [67].

$S(3,4,22) ; b=385$. There are exactly 21 incquivalent cyclic designs, and exactly 5 disjoint cyclic designs [59]. At least 11 disjoint dcsigns exist [144], [145].

$S(5,6,24) ; b=7084$. See Table XV. $\Lambda t$ least three inequivalent dcsigns exist [56], [74].

$S(5,8,24) ; b=759$. The weight 8 words in the $[24,12,8]$ Golay code Unique [182], [183], [132], [45]. At least 9 disjoint designs exist [114] $S(2,5,25)=A G(2,5) ; b=30$. Unique.

$S(3,4,26) ; b=210$. See Theorem 5 or [81]. At least 13 disjoint designs exist [146].

$S(3,6,26) ; b=130$. Uniquc (sce Theorem 13, Table XI. [33])

$S(2,3,27) ; b=117$. Many examples, one of which is $A G(3,3)$

$S(2,4,28) ; b=63$. See [52], [84], Theorem 13. At least 154 inequivalent designs exist [24], [26]

$S(3,4,28) ; b=819$ [81]. At least 18 disjoint designs exist [66]

$S(5,7,28) ; b=4680$. See $[56]$ or Table XV.

$S(2,6,31)=P G(2,5) ; b=31$. Cyclic: $\{0,1,3,8,12,18)$ mod 31 ; unique

Furthermore (31) can be rcplaced by

$$
A(n, 4, w) \geq \max _{k=0,1, \cdots, n-1}\left|C_{k}^{(w)}\right|,
$$

although in practice this gives little improvement on (31).

Kløve [110] generalized Theorem 1 by replacing $\mathbb{Z}_{n}$ with an arbitrary abelian group $G$ of order $n$, defining $f$ by

$$
f(a)=\sum_{g \in G} a_{g} g
$$

He found an explicit formula for the bcst lower bound for $A(n, d, w)$ (for given $n$ and $w$ ) obtained from (34) using the optimal choice of $G$. Again these results do not greatly differ from those obtained from (31). See also Delsarte and Piret [50].

For $d \geq 6$ there are several competing analogues of Theorem 14. 
Theorem 15 ([72]): If $\delta \geq 3$ and $n$ is a prime power then

$$
A(n, 2 \delta, w) \geq \frac{1}{n^{\delta-1}}\left(\begin{array}{l}
n \\
w
\end{array}\right) .
$$

Proof: Let $n=q$ be a prime power, and write $F_{q}=$ $\left\{\alpha_{0}, \cdots, \alpha_{a-1}\right\}$. The proof replaces (32) by the map $f$ from $\mathbb{\top}_{2}^{n}$ to $\mathrm{F}_{q}^{\delta-1}$ given by

$$
f(a)=\left(T_{1}(a), T_{2}(a), \cdots, T_{\delta-1}(a)\right),
$$

wherc

$$
\begin{gathered}
T_{1}(a)=\sum_{a_{i}=1} \alpha_{i}, \\
T_{2}(a)=\sum_{\substack{i<j \\
a_{i}=a_{j}=1}} \alpha_{i} \alpha_{j}, \\
T_{3}(a)=\sum_{\substack{i<j<k \\
a_{i}=a_{j}-a_{k}-1}} \alpha_{i} \alpha_{j} \alpha_{k}, \\
\ldots . .
\end{gathered}
$$

For any $\boldsymbol{k} \in \mathbb{F}_{q^{-1}}^{\delta-1}$ it can be shown that the code

$$
C_{k}^{(w)}=\{a \in \underset{2}{\sim}: f(a)=k, \quad w t(a)=w\}
$$

has minimal distance $2 \delta$, and (36) follows.

A subset $S=\left\{s_{1}, \cdots, s_{n}\right\}$ of an abelian group $G$ is called an $S_{t}$-set of size $n$ if all the sums

$$
s_{i_{1}}+s_{i_{2}}+\cdots+s_{i_{1}}
$$

for $1 \leq i_{1}<i_{2} \cdots<i_{t} \leq n$ are distinct in ( $i$ (cf. [72], [73], [75], [76]). $S_{t}$-sets are relevant here because of the following result.

Theorem 16 (Compare [72]): If there exists an $S_{\delta-1}$-set of size $n$ in an abelian group of order $m$ then

$$
A(n, 2 \delta, w) \geq \frac{1}{m}\left(\begin{array}{c}
n \\
w
\end{array}\right) .
$$

Proof: Replace (35) by $f(a)=\sum_{i=1}^{n} s_{i} a_{i}$.

In 1962 Bosc and Chowla ([17], [76]; see also [72]) constructed an $S_{t}$-set of size $q+1$ in $\mathbb{Z}_{m}$ for $m=\left(q^{t+1}-\right.$ 1) $/(q-1)$, for any prime power $q$. Another construction is the following.

Theorem 17: For any prime power $q$ there is an $S_{t}$-set of size $q$ in $\mathbb{Z}_{m}$ for $m=q^{t}-1$.

Proof: Let $\xi$ be a primitive element of the field of order $q^{t}$, and let $F$ be the subfield of order $q$. The set $\left\{s \in \mathbb{Z}_{m} \mid \xi^{s}-\xi \in F\right\}$ is the desired $S_{t}$-set.

Furthermore the columns of a parity-check matrix for an $[n, k, d=2 t+1]$ binary linear code, together with the zero vector, form an $S_{t}$-set of size $n+1$ in the abelian group $\mathbb{Z}_{2}^{n-k}$. (The corresponding constant weight code is

\begin{tabular}{|c|c|c|c|c|c|}
\hline$n$ & $m$ & $n$ & $m$ & $n$ & $m$ \\
\hline 2 & $2^{a \prime}$ & 11 & yge & 20 & $381^{h}$ \\
\hline 3 & $3^{\prime \prime}$ & 12 & $123 f^{\prime}$ & 21 & $?$ \\
\hline 4 & $6^{a}$ & 13 & $152^{\prime \prime}$ & 22 & $?$ \\
\hline 5 & $11^{\prime \prime}$ & 14 & $183^{h}$ & 23 & ? \\
\hline 0 & $16^{b}$ & 15 & $222^{i}$ & 24 & $512^{\prime}$ \\
\hline 7 & 24 & 16 & $255^{\prime}$ & 25 & 624 \\
\hline 8 & $40^{\prime \prime}$ & 17 & $?$ & 26 & $651^{h}$ \\
\hline 9 & $52^{d}$ & 18 & $256^{k}$ & 27 & 728 \\
\hline 10 & $72^{a}$ & 19 & $360^{i}$ & 28 & $757^{h}$ \\
\hline
\end{tabular}
the collection of words of weight $w$ in some coset of the code extended with an (anti-) parity check bit.)

Let $v_{\mathbb{Z}}(n)$ denote the smallest $m$ such that an $S_{2}$-set of size $n$ exists in $\mathbb{Z}_{m}$, and more generally let $\varepsilon(n)$ be the smallest $m$ such that an $S_{2}$-sct of size $n$ exists in some
TABLE V

UPPER Bolinds FOR $S_{2}$-SETS

abclian group of order $m$. In [73] it is shown that $v_{\text {" }}(n) \sim$ $n^{2}$ as $n \rightarrow \infty$, but for $\iota(n)$ it is known only that

$$
\left(\begin{array}{l}
n \\
2
\end{array}\right) \leq l(n)<n^{2}+O\left(n^{36 / 23}\right)
$$

Table $\mathrm{V}$ gives the best upper bounds presently known on $v(n)$ for $2 \leq n \leq 28$.

Key to Table $\mathrm{V}^{\prime}$

Elements of the elementary abelian groups $\mathbb{Z}_{2}^{k}$ are written in decimal; for example (0)(1) $\in \mathbb{Z}_{2}^{4}$ is written as 5 .

$a=$ Optimal $S_{2}$-set in $\mathbb{Z}_{m}$ (see Table IV in [73]).

$b=\operatorname{In} \mathbb{Z}_{2}^{4}$ usc $\mathbf{0}$ and columns of parity-check matrix for $[5,1,5]$ code

$c=\{(0,()),(1,0),(2,0),(4,0),(0,1),(7,1),(0,2)\}$ in $\mathbb{Z}_{2}^{3} \times$ $\mathbb{Z}_{3}$.

$d=\{(0,0),(0,1),(0,2),(1,0),(1,4),(1,8),(2,2),(2,5)$, $(2,8)\}$ in $\mathbb{Z}_{2}^{2} \times \mathbb{Z}_{1,3}$.

$e=\{0,1,2,4,7,15,26,45,54,66,83\}$ in $\mathbb{Z}_{44}$.

$f=\{0,1,2,4,7,14,23,31,48,59,74,92\}$ in $\mathbb{Z}_{123}$.

$g=\{0,1,2,4,7,12,20,35,63,77,106,115,132\}$ in $\mathbb{Z}_{152}$.

$h=$ Perfect difference set [8].

$i=\{0,1,2,4,7,12,20,29,46,69,92,116,140,170,191\}$ in $\mathbb{Z}_{222}$.

$j=$ Theorem 17 .

$k=$ In $\mathbb{Z}_{2}^{8}$ use $\mathbf{0}$ and columns of parity-check matrix for $[17,9,5]$ cyclic code.

$l=$ In $\mathbb{Z}_{2}^{9}$ use $\mathbf{0}$ and columns of parity-check matrix for Wagner's $[23,14,5]$ code [179].

For example, when $n=25$, Theorem 16 and Table $\mathrm{V}$ imply that

$$
A(25,6,12) \geq\left\lceil\frac{1}{624}\left(\begin{array}{l}
25 \\
12
\end{array}\right)\right\rceil=8334 \ddagger .
$$

( $¥$ indicates a code which does not yicld thc best lower bound known for this value of $A(n, d, w)$, but is easy to construct.) The entries labeled " $g s$ " in Table I are obtained in this way. The cntries in the third column of Table $\mathrm{V}$ appear quite weak, and the construction of better $S_{2}$-sets would probably improve several entries of Table I-B.

We also mention an unpublished gencral lower bound of Zaptcioglu [184], although in the range of our tables it 
does not lead to any new bounds. Suppose $C$ attains $A(n, d, w)=M$, where $d=2 \delta$. A d-neighbor of $c \in C$ is any $\iota: \in \mathbb{F}_{2}^{n}$ such that $w t(v)=w$ and $\operatorname{dist}(v, c)<d$. Let $G N(n, d, w, i)$ denote the number of $d$-neighbors of the $i$ th word of $C$ that are also $d$-neighbors of the $j$ th word of $C$ lor some $j<i$. Then Zaptcioglu begins with the equality

$$
A(n, d, w)=\frac{\left(\begin{array}{l}
n \\
w
\end{array}\right)+\sum_{i=2}^{M} G N(n, d, w, i)}{\sum_{i=0}^{\delta-1}\left(\begin{array}{c}
w \\
i
\end{array}\right)\left(\begin{array}{c}
n-w \\
i
\end{array}\right)}
$$

This is proved by multiplying both sides by the denominator, and using a straightforward counting argument. Since $G N \geq 0$ we immediately obtain the "Gilbert bound" of [72]:

$$
A(n, d, w) \geq G(n, d, w),
$$

where

$$
G(n, d, w)=\frac{\left(\begin{array}{l}
n \\
w
\end{array}\right)}{\sum_{i=0}^{\delta-1}\left(\begin{array}{c}
w \\
i
\end{array}\right)\left(\begin{array}{c}
n-w \\
i
\end{array}\right)} .
$$

Zaptcioglu shows that (38) has the following stronger conscquencc.

Theorem 18 (Zaptcioglu /184]): For $d \geq 4$ and $w \geq 3$ we have

$$
\begin{gathered}
A(n, d, w) \geq G(n, d, w)+\frac{\sum_{i=0}^{\delta-1}\left(\begin{array}{c}
w \\
i
\end{array}\right)\left(\begin{array}{c}
n-w-1 \\
i
\end{array}\right)}{\sum_{i=0}^{\delta-1}\left(\begin{array}{c}
w \\
i
\end{array}\right)\left(\begin{array}{c}
n-w \\
i
\end{array}\right)} \\
\cdot[A(n-1, d, w)-G(n-1, d, w)] .
\end{gathered}
$$

\section{Vi. The Partitionisig Constrlction}

The partitioning construction, used by several authors ([13], [49], [68], [149], [150]), produces good lower bounds for codes with minimal distance 4 . It is related to a generalization of Theorem 14 (see subsection 4).

A partition $\Pi(n, w)=\left(X_{1}, \cdots, X_{m}\right)$ is a collection of disjoint sets or classes $X_{1}, \cdots, X_{m}$, each of which is a code of length $n$, distance 4 and constant weight $w$, and whose union contains all $\left(\begin{array}{c}n \\ w\end{array}\right)$ vectors of weight $w$. The vector $\pi(n, w)=\left(\left|X_{1}\right|, \cdots,\left|X_{n}\right|\right)$ with integer components is the index lector of the partition $\operatorname{II}(n, w)$, and

$$
\pi(n, w) \cdot \pi(n, w)=\sum_{i=1}^{m}\left|X_{i}\right|^{2}
$$

is its norm. We always assume $\left|X_{1}\right| \geq \cdots \geq\left|X_{m}\right|$. When there are several different partitions available for a given $n$ and $w$ we of ten denote them by $\Pi_{1}(n, w), \Pi_{2}(n, w), \cdots$, and their index vectors by $\pi_{1}(n, w), \pi_{2}(n, w), \cdots$.

The direct product $\Pi\left(n_{1}, w_{1}\right) \times \Pi\left(n_{2}, w_{2}\right)$ of two partitions $\left(X_{1}, \cdots, X_{m_{1}}\right),\left(Y_{1}, \cdots, Y_{m_{2}}\right)$ consists of the vectors

$$
\left\{(u, v): u \in X_{i}, \quad v \in Y_{i}, \quad 1 \leq i \leq m\right\}
$$

where $m=\min \left\{m_{1}, m_{2}\right\}$. This set (which is only part of the final code) clearly has length $n_{1}+n_{2}$, distance 4 , weight $w_{1}+w_{2}$, and contains

$$
\pi\left(n_{1}, w_{1}\right) \cdot \pi\left(n_{2}, w_{2}\right)=\sum_{i=1}^{m}\left|X_{i}\right|\left|Y_{i}\right|
$$

words.

The construction: To obtain a code of length $n$, distance 4 and weight $w$ by the partitioning construction wc write $n=n_{1}+n_{2}$, choose $\epsilon=0$ or 1 , and take the union of the dircct products

$$
\begin{gathered}
\Pi\left(n_{1}, \epsilon\right) \times \Pi\left(n_{2}, w-\epsilon\right), \\
\Pi\left(n_{1}, \epsilon+2\right) \times \Pi\left(n_{2}, w-\epsilon-2\right), \\
\Pi\left(n_{1}, \epsilon+4\right) \times \Pi\left(n_{2}, w-\epsilon-4\right), \\
\cdots .
\end{gathered}
$$

It is apparent that this codc does have the required propertics, and contains

$$
\begin{aligned}
\pi\left(n_{1}, \epsilon\right) \cdot \pi( & \left.n_{2}, w-\epsilon\right)+\pi\left(n_{1}, \epsilon+2\right) \cdot \pi\left(n_{2}, w-\epsilon-2\right) \\
& +\pi\left(n_{1}, \epsilon+4\right) \cdot \pi\left(n_{2}, w-\epsilon-4\right)+\cdots
\end{aligned}
$$

words.

As an illustration we construct a code showing that $A(18,4,7) \geq 2041$. We take $n_{1}=8, n_{2}=10, \epsilon=1$ and form the union of the direct products

$$
\begin{aligned}
& \Pi(8,1) \times \Pi_{2}(10,6), \\
& \Pi(8,3) \times \Pi_{1}(10,4), \\
& \Pi(8,5) \times \Pi(10,2), \\
& \Pi(8,7) \times \Pi(10,0),
\end{aligned}
$$

(see below and Table VI). The corresponding index vectors are

$$
\begin{aligned}
\pi(8,1) & =(1,1,1,1,1,1,1,1), \\
\pi(10,6) & =(30,30,30,30,26,25,22,15,2),
\end{aligned}
$$

so the first direct product contains

$1 \cdot 30+1 \cdot 30+1 \cdot 30+1 \cdot 30+1 \cdot 26+1 \cdot 25$

$$
+1 \cdot 22+1 \cdot 15=208
$$

words;

$$
\begin{aligned}
\pi(8,3) & =(8,8,8,8,8,8,8), \\
\pi_{1}(10,4) & =(30,30,30,30,30,22,22,12,2,2),
\end{aligned}
$$

so the second direct product contains

$$
8 \cdot 30+\cdots+8 \cdot 30+8 \cdot 22+8 \cdot 22=1552
$$

words;

$$
\begin{aligned}
\pi(8,5) & =(8,8,8,8,8,8,8), \\
\pi(10,2) & =(5,5,5,5,5,5,5,5,5),
\end{aligned}
$$

so the third direct product contains

$$
8 \cdot 5+\cdots+8 \cdot 5=280
$$


words; and

$$
\begin{aligned}
\pi(8,7) & =(1,1,1,1,1,1,1,1), \\
\pi(10,0) & =(1),
\end{aligned}
$$

so the last direct product contains $1 \cdot 1=1$ word. The total number of codewords is

$$
208+1552+280+1=2041
$$

Codes obtained from the partitioning construction are indicated by " $p(0), \cdots, " p 3$ " in Table I-A. The values of $n_{1}, n_{2}, \epsilon$ are as follows:

$\begin{array}{lll}\text { for type } p(), & n_{1}=\left[\begin{array}{l}n \\ 2\end{array}\right], \quad n_{2}=n-n_{1}, \quad \epsilon=0, \\ \text { for type } p 1, & n_{1}=\left[\begin{array}{l}n \\ 2\end{array}\right], \quad n_{2}=n-n_{1}, \quad \epsilon=1, \\ \text { for typc } p 2, & n_{1}=\left[\begin{array}{l}\frac{n}{2} \\ \text { for }\end{array}\right]-1, \quad n_{2}=n-n_{1}, \quad \epsilon=0, \\ \text { for type } p 3, & n_{1}=\left[\begin{array}{c}n \\ 2\end{array}\right]-1, \quad n_{2}=n-n_{1}, \quad \epsilon=1 .\end{array}$

The partitions needed are listed in Table VI. (We do not take the space to indicatc the particular partitions used in each construction.) Partitioning also gives $A(16,4,5) \geq$ 305 †.

We next discuss the choice of a good partition. When applying the partitioning construction in a situation where several different partitions are available, we see from (39), (41) that we should choose pairs $\Pi\left(n_{1}, w_{1}\right), \Pi\left(n_{2}, w_{2}\right)$ so as to maximize the inner product $\pi\left(n_{1}, w_{1}\right) \cdot \pi\left(n_{2}, w_{2}\right)$. (For example we use $\Pi_{2}(10,6)$ rather than $\Pi_{1}(10,6)$ in the first line of (42), so as to maximize the inner product with $\pi(8,1)=(1,1,1,1,1,1,1,1)$.)

We say that one partition $\Pi\left(n_{1}, w_{1}\right)$ dominates another $\Pi^{\prime}\left(n_{1}, w_{1}\right)$ if

$$
\pi\left(n_{1}, w_{1}\right) \cdot \pi\left(n_{2}, w_{2}\right) \geq \pi\left(n_{1}, w_{1}\right) \cdot \pi\left(n_{2}, w_{2}\right)
$$

holds for all choices of $n_{2}, w_{2}$ and all possible index vectors $\pi\left(n_{2}, w_{2}\right)$. If a partition is dominated it necd never be uscd in the construction.

There is a simple test for dominance.

Theorem 19: $\pi\left(n_{1}, w_{1}\right)=\left(a_{1}, \cdots, a_{m}\right)$ dominates $\pi^{\prime}\left(n_{1}, w_{1}\right)=\left(b_{1}, \cdots, b_{m}\right)$ if and only if

$$
\sum_{i=1}^{j} \boldsymbol{a}_{i} \geq \sum_{t=1}^{j} b_{i}, \quad \text { for all } j=1, \cdots, \max \left\{m, m^{\prime}\right\} .
$$

Proof: The components of the index vector $\pi\left(n_{2}, w_{2}\right)$ in (43) are nonincreasing positive integers, and any such vector is a positive combination of vectors of the form $(1,1, \cdots, 1,0, \cdots, 0)$.

A partition $\Pi(n, w)$ is optimal if it dominates all other $\Pi^{\prime}(n, w)$ with the same $n$ and $w$, or just maximal if it is not itsclf dominated by any other $\Pi^{\prime}(n, w)$.

In the remainder of this section we describe techniques for linding good partitions.

1) By taking complements the cxistence of a $\Pi(n, w)$ implies the existence of a $\Pi(n, n-w)$ with the same index vector.
2) For $w=0$ and 1 there are trivial partitions with index vectors

$$
\begin{aligned}
& \pi(n, 0)=(1), \\
& \pi(n, 1)=(1,1, \cdots,(n \text { times })),
\end{aligned}
$$

and for $w=2$ there arc well-known partitions

$$
\begin{aligned}
& \pi(n, 2)=\left(\frac{n}{2}, \frac{n}{2}, \cdots,(n-1 \text { times })\right), n \text { even, } \\
& \pi(n, 2)=\left(\frac{n-1}{2}, \frac{n-1}{2}, \cdots,(n \text { times })\right), n \text { odd }
\end{aligned}
$$

see [136]. All these partitions arc optimal.

3) If we have a lower bound $A(n, 4, w) \geq M$ there is always the partition

$$
\pi(n, w)=\left(M, 1,1,1, \cdots,\left(\left(\begin{array}{l}
n \\
w
\end{array}\right)-M \text { times }\right)\right) .
$$

(This is useful when the inner product with $\pi(n, \mathbf{0})=(1)$ is to be maximized.)

4) The results of [72]-see Theorcm 14-show that a partition $\Pi(n, w)$ always exists with $m \leq n$ classes. In many cascs-for example if $n$ is prime-the index vector for this partition is

$$
\pi(n, w)=\left(\frac{1}{n}\left(\begin{array}{l}
n \\
w
\end{array}\right), \frac{1}{n}\left(\begin{array}{l}
n \\
w
\end{array}\right), \cdots,(n \text { times })\right) .
$$

5) A number of optimal partitions with $w=3$ are available in the literature. It is known that, if $n \equiv 1$ or 3 $(\bmod 6), n \neq 7$, then the set of all $\left(\begin{array}{l}11 \\ 3\end{array}\right)$ triples can be partitioned into $n-2$ mutually disjoint Stciner triple systems-implying that there is an optimal partition

$$
\pi(n, 3)=\left(\frac{n(n-1)}{6}(n-2 \text { times })\right) .
$$

This result is due to Lu [130], [131]. (The manuscript of [131] was incomplete at the timc of the author's death, but the six unfinished values of $n$ have since been dealt with by Teirlinck [174].) Earlier results on this problem were given by Cayley [32], Denniston [54], [55], [57], Kirkman [109], Phelps [143], Schreiber [158], Teirlinck [171], Wilson [181]. When (as in this case) the set of all $\left(\begin{array}{c}n \\ w\end{array}\right)$ vectors of weight $w$ can be partitioned into disjoint designs all having the same parameters, the designs arc said to form a "large set." Further results on partitions may be found in [4], [19], [60], [65]-[68], [85], [113], [119], [144], [146], [159]-[162], [172], [173].

6) Van Pul [149], [150] and Etzion-Van Pul [68] found the partitions $\Pi(6,3), I I(7,3), \Pi(8,4), \Pi(10,3), \Pi_{1}(10,4)$ mentioned in Table VI. (However only $\Pi_{1}(10,4)$ is given explicitly in [68].)

7) In situations not covered by the preceding comments we use the computer to find good partitions. Our methods are based on the following considerations. a) Finding a good partition is a graph coloring problem. For if we construct the graph whose vertices represent $\left(\begin{array}{c}n \\ w\end{array}\right)$ binary vectors of weight $w$, and join two vertices by an edge if 
and only if the vertices are Hamming distance 2 apart, then a partition $\Pi(n, w)=\left(X_{1}, \cdots, X_{m}\right)$ describes a coloring of the vertices using $m$ colors, the classes $X_{1}, \cdots, X_{m}$ being the color classes. b) A useful heuristic for finding a good partition is to maximize the norm of the index vector. This is only a heuristic, for we already saw in the previous example that there are situations wherc partitions with less than the maximal norm are preferable. However, a partition with the greatest possible norm is always maximal. A second heuristic is to minimize the number of color classes. c) Good methods of choosing the initial classes $X_{1}, X_{2}, \cdots$ of $\Pi(n, w)$ are to use a maximal independent set algorithm, to use as many disjoint Steiner systems as possible (see Tables IV), or more generally to look for as many disjoint (or almost disjoint) copies as possible of the largest known code of length $n$ and weight $w$. (Some partition obtained by repeatedly removing maximal independent sets is maximal.) We then look for a coloring of the remaining vertices with the greatest norm.

David Johnson [98] has developed a simulated annealing program for graph coloring, which attempts to maximize the sum of the squares of the color class sizes. Some of the partitions given in Table VI below were found (in part) using this program. Others were found by various iterative procedures. Johnson's program uses Kempechain interchanges for graph coloring; a recent alternative suggestion by Berge [10] may lead to better colorings and hence better partitions.

8) The methods of 7) are only successful for $n$ up to about 14. For $n \geq 12$ we also made use of Etzion and Van Pul's "Construction B" for combining partitions ([68]). This construction works as follows. Given partitions $\mathrm{II}(l, u)$ and $\Pi(m, v)$ (for all $u, 0 \leq u \leq \min (l, w)$ and $v$, $0 \leq l \leq \min (m, w))$ we construct a partition $\Pi(n, w)$, where $n=l+m$, by repeatedly using the partitioning construction (cf. (40)). Morc precisely, start with two sufficiently long rows of empty buckets-the "odd" row and the "even" row. For each pair $(u, v)$ with $u+v=w$ we consider the given partitions $\Pi(l, u)=\left(X_{1}, \cdots, X_{r_{1}}\right)$ and $\Pi(m, v)=\left(Y_{1}, \cdots, Y_{r_{2}}\right)$, and distribute the $r_{1} r_{2}$ direct

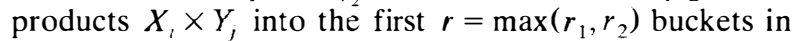
the row with the same parity as $u$, where each bucket gets $\min \left(r_{1}, r_{2}\right)$ codes $X_{i} \times Y_{j}$, such that no bucket contains two codes $X_{i} \times Y_{j}$ and $X_{i^{\prime}} \times Y_{j^{\prime}}$ where $i=i^{\prime}$ or $j=j^{\prime}$. The result will be that the buckets form a partition $\Pi(n, w)$. (At least $r$ buckets are requircd because of the conditions $i \neq i^{\prime}$ and $j \neq j^{\prime}$. A distribution into $r$ buckets is possible because the complete bipartite graph $K_{r_{1}, r_{2}}$ has an edge coloring with $r$ colors.) For example, take $(n, w)=(12,4)$. Using partitions with index vectors $\pi(6,0)=\pi(6,6)=(1)$, $\pi(6,1)=\pi(6,5)=(1,1,1,1,1,1), \pi(6,2)=\pi(6,4)=$ $(3,3,3,3,3), \pi(6,3)=(4,4,4,4,2,2)$ we fill five buckets in the even row with $1 \cdot 3+5 \cdot 3 \cdot 3+3 \cdot 1=51$ words each, and six buckets in the odd row with $\pi(6,1) \cdot \pi(6,3)+\pi(6,3)$. $\pi(6,1)=40$ words each, to obtain a partition $\Pi(12,4)$ with index vector $(51,51,51,51,51,40,40,40,40,40,40)$.

Table VI gives the index vectors of the nontrivial partitions used in constructing the codes marked " $p 0$," " $p 1$,"
" $p 2$," " $p 3$ " in Table I. In view of 1 ) we only give partitions $\Pi(n, w)$ with $w \leq n / 2$. The partitions marked "A" are given explicitly in the Appendix. Partitions known to be optimal are marked with an asterisk. For some valucs of $n$ and $w$ several different partitions $\Pi_{i}(n, w) \quad(i=$ $1,2, \cdots)$ are given ( $i$ is given in column 3$)$, no one of which dominates any of the others. In many cases we have found other partitions besides those in Table VI, which although not needed for the partitioning construction, neverthcless are not dominated by the partitions in the table. It would be nice to replace these with smaller sets of maximal partitions. For $\Pi(10,4)$ there are at least two maximal partitions, and, as will be shown below, certainly no optimal $\Pi(10,4)$ exists.

\section{Key to Table VI}

$* \quad=$ Optimal.

$\Lambda=$ See Appendix.

$\mathrm{B}=$ Construction $\mathrm{B}$.

$\mathrm{GS}=$ From Theorem 14 .

(1) $=$ Shortened $\Pi(9,3)$

(2) $=$ Shortened $\Pi(10,4)$.

(3) $=$ Shortened $\Pi(13,3)$.

(4) $=$ Shortened $\Pi(15,3)=(35,35, \cdots,(13$ times $))$ of [55]

Optimal and maximal partitions: We first show that the partitions marked with an asterisk in Table VI are optimal. Obviously $\pi(n, w)$ is optimal if all parts (except perhaps the smallest) have size $A(n, 4, w)$, which proves the optimality of $\pi(8,3), \pi(9,3)$, etc.

$$
\pi(6,3)=(4,4,4,4,2,2) \text { is optimal. }
$$

Proof: Any code attaining $A(6,4,3)=4$ is equivalent to

$$
C_{1}=\left[\begin{array}{llllll}
1 & 1 & 1 & 0 & 0 & 0 \\
1 & 0 & 0 & 1 & 1 & 0 \\
0 & 1 & 0 & 1 & 0 & 1 \\
0 & 0 & 1 & 0 & 1 & 1
\end{array}\right] .
$$

We must show that $(4,4,4,4,4)$ and $(4,4,4,4,3,1)$ are impossible. Since $C_{1}$ has column sums 2 , the last four vectors of the partition also have column sums 2 , and are therefore equivalent either to $C_{1}$ or to

$$
C_{2}=\left[\begin{array}{llllll}
1 & 1 & 1 & 0 & 0 & 0 \\
1 & 1 & 0 & 1 & 0 & 0 \\
0 & 0 & 1 & 0 & 1 & 1 \\
0 & 0 & 0 & 1 & 1 & 1
\end{array}\right] .
$$

The vectors of $C_{2}$ cannot be partitioned $3+1$, so the last four vectors of the partition are also equivalent to $C_{1}$, and the partition is $(4,4,4,4,4)$. Let the first class bc $C_{1}$. Then the four vectors $100011,100101,110001,101001$ have mutual distances 2 and each lies in a different one of the other four classes. It is now easy to check by hand that these classes cannot be completed.

$$
\pi(7,3)=(7,7,6,6,5,4) \text { is optimal. }
$$

Proof: We know from Table IV that there do not exist three classes of size 7 . Also $(7,7,6,6,6,1,1,1)$ is 
TABLE VI

Nontrivinl Partitions lsed to Construct Confs in Table I

\begin{tabular}{|c|c|c|c|c|c|c|}
\hline$n$ & $w^{\prime}$ & $i$ & $m$ & Norm & Source & Index Vector of $\prod_{i}(n, w)$ \\
\hline 6 & 3 & 1 & 6 & 72 & $A^{*}$ & 4.4 .4 .4 .2 .2 \\
\hline 7 & 3 & 1 & 6 & 211 & $A^{*}$ & 7.7.6.6.5.4 \\
\hline 8 & 3 & 1 & 7 & 448 & $(1)^{*}$ & 8.8 .8 .8 .8 .8 .8 \\
\hline 8 & 4 & 1 & 6 & 844 & $A^{*}$ & 14.14 .12 .12 .10 .8 \\
\hline 9 & 3 & 1 & 7 & 1008 & Table IV* & 12.12 .12 .12 .12 .12 .12 \\
\hline 9 & 4 & 1 & 8 & 2066 & A & 18.18 .18 .18 .16 .15 .15 .8 \\
\hline$y$ & 4 & 2 & 10 & 2036 & (2) & $18.18,18,18.18,14.13 .7 .1,1$ \\
\hline 10 & 3 & 1 & 10 & 15.30 & $A^{*}$ & $13.13,1,3,1,3,1.3,1,3,1,3,1.3,1,3,3$ \\
\hline 10 & 4 & I & 10 & 5620 & {$[68]$} & 30.30 .30 .30 .30 .22 .22 .12 .2 .2 \\
\hline 10 & 4 & 2 & 9 & 5614 & A & $30,30,30,30,26,25,22,15.2$ \\
\hline 10 & 5 & 1 & 8 & 8044 & A & $36.36,34.34,29,29,27.27$ \\
\hline 11 & 3 & 1 & 10 & 2731 & A & $17.17 .17 .17 .17 .17 .17 .16,16,14$ \\
\hline 11 & 3 & 2 & 11 & 271.3 & A & 17.17 .17 .17 .17 .17 .17 .17 .16 .12 .1 \\
\hline 11 & 3 & 3 & 11 & 2705 & A & 17.17 .17 .17 .17 .17 .17 .17 .17 .10 .2 \\
\hline 11 & 4 & 1 & 11 & 10724 & A & $35,35,35, .34,3,3,3,3,3,3,32, .31,25.4$ \\
\hline 11 & 4 & 2 & 11 & 10616 & A & $35,35,35,35,35,33,3,3,32,28,21.8$ \\
\hline 11 & 5 & 1 & 10 & 25066 & A & $66.66,60,60.54,45,44.40,26,1$ \\
\hline 11 & 5 & 2 & 10 & 25046 & A & $66,66,60,60.54,45,44,42,22,3$ \\
\hline 12 & 3 & 1 & 11 & 4400 & $(3)^{*}$ & $20,20,20,20,20,20,20,20,20,20,20$ \\
\hline 12 & 4 & 1 & 11 & 2290.3 & A & $51.51 .51, .51 .51,46,45,44.44,34.27$ \\
\hline 12 & 4 & 2 & 12 & 2284.3 & A & $51.51 .51 .51,49,48,48,42,42.37,23.2$ \\
\hline 12 & 4 & 3 & 12 & 22815 & A & 51.51 .51 .51 .49 .48 .48 .42 .42 .40 .15 .7 \\
\hline 12 & 4 & 4 & 12 & 22795 & A & $51.51 .51 .51 .49 .48 .46 .44,43,37.20 .4$ \\
\hline 12 & 4 & 5 & 12 & 22755 & A & $51.51 .51 .51 .49 .48,48,45,39.36,22,4$ \\
\hline 12 & 4 & 6 & 12 & 2266.3 & A & $51.51 .51 .51 .49 .48 .48 .45 .41,32.22 .6$ \\
\hline 12 & 5 & 1 & 12 & 55860 & A & $80.80 .80,80.72 .70 .69,67.67 .62 .48 .17$ \\
\hline 12 & 5 & 2 & 1.3 & 55.350 & A & $80,80,80,80,75,72,71,69,6,3,55,40,23,4$ \\
\hline 12 & 6 & 1 & 10 & 99952 & A & $1.32,1,32,120,120.110,94,90,76,36,14$ \\
\hline 12 & 6 & 2 & 10 & 99776 & A & 1.32 .132 .120 .120 .110 .94 .90 .72 .42 .12 \\
\hline 12 & 6 & 3 & 10 & 99072 & A & $1.32 .132 .120 .110 .110 .97 .91,75.47 .10$ \\
\hline 1.3 & 3 & 1 & 11 & 74.36 & Table IV* & $26,26,26,26.26,26,26,26,26,26,26$ \\
\hline 1.3 & 4 & 1 & 1.3 & 42165 & A & $65,65.65,65.62,61,60,57.57,53.52 .45 .8$ \\
\hline 1.3 & 4 & 2 & 1.3 & 42163 & A & $65,65,65,65,62,61,60,58,55,54,52,45,8$ \\
\hline 1.3 & 4 & 3 & 1.3 & 42147 & A & $65,65.65,65,62,60,60,58.57 .54,49.47 .8$ \\
\hline 13 & 4 & 4 & 1.3 & 42015 & A & $65,65.65 .65 .62 .60,60.58 .57 .56 .53,37.12$ \\
\hline 1.3 & 4 & 5 & 1.3 & 41975 & A & $65.65,65,65,62.62 .60 .59,56,55.49,40,12$ \\
\hline 1.3 & 4 & 6 & 13 & 41795 & A & $65,65.65,65,62.61,61, .59 .58,54,50, .32,18$ \\
\hline 1.3 & 5 & 1 & 1.3 & 1.35679 & A & $123.123 .121 .115 .110,109,109,102.99,92.84 .72 .28$ \\
\hline 1.3 & 5 & 2 & 1.3 & 13.5557 & A & $123.123,121.115 .110 .109,109,101.99,9.3 .86 .68 .30$ \\
\hline 13 & 5 & 3 & 14 & 1.354 .37 & A & 123.122 .121 .114 .110 .109 .109 .102 .97 .91 .85 .77 .26 .1 \\
\hline 1.3 & 5 & 4 & 1.3 & 1.34757 & A & 123.123 .123 .116 .110 .109 .106 .100 .98 .92 .81 .68 .38 \\
\hline 1.3 & 5 & $s$ & 14 & 1.34753 & A & 12.3 .12 .3 .123 .116 .110 .109 .107 .104 .97 .89 .83 .62 .40 .1 \\
\hline 1.3 & 6 & 1 & 14 & 239106 & A & $166.166 .160 .156 .143,14,3,1,39.135,131,122,107.100 .46 .2$ \\
\hline 1.3 & 6 & 2 & 14 & 2.39082 & A & $166.166 .160 .156 .144 .142 .1,38,1,37,1.31 .120,106,102.46 .2$ \\
\hline 1.3 & 6 & 3 & 13 & 2388.32 & A & $166.166,160,156,14.3,142.138 .136 .130 .120 .111 .97 .51$ \\
\hline 1.3 & 6 & 4 & 14 & 2.38698 & A & 166.166 .160 .156 .145 .142 .139 .136 .131 .118 .113 .91 .5() .3 \\
\hline 1.3 & 6 & 5 & 14 & 2.38 .384 & A & 166.166 .160 .156 .145 .142 .139 .136 .131 .119 .112 .88 .52 .4 \\
\hline 1.3 & 6 & 6 & 1.3 & 238116 & A & $166.166 .160,156,145,144.137 .132 .127 .118 .111 .98 .56$ \\
\hline 1.3 & 6 & 7 & 14 & 237556 & A & 166.166 .160 .156 .145 .142 .140 .136 .131 .118 .106 .86 .59 .5 \\
\hline 14 & 3 & 1 & 1.3 & 10192 & $(4)^{*}$ & $28,28,28,28,28,28,28,28,28,28,28,28,28$ \\
\hline 14 & 4 & 1 & 1.3 & 79.393 & A & $91.91 .91 .91,81.79 .78 .77 .74,73.71 .62,42$ \\
\hline 14 & 4 & 2 & 14 & 79.357 & A & 91.91 .91 .91 .80 .79 .78 .78 .75 .74 .71 .60 .41 .1 \\
\hline 14 & 4 & 3 & 14 & 793.39 & A & $91.91 .91 .91 .81 .79,79.77 .76 .71,67.67 .38 .2$ \\
\hline 14 & 4 & 4 & 1.3 & 79269 & A & 91.91 .91 .91 .82 .79 .78 .77 .75 .72 .67 .62 .45 \\
\hline 14 & 5 & 1 & 1.5 & 291280 & A & 169.169 .165 .156 .156 .152 .149 .144 .143 .137 .134 .121 .118 .80 .9 \\
\hline 14 & 5 & 2 & 15 & 290646 & A & $169.169 .165 .156,155.153,151.147 .143 .137 .134 .120 .112 .76 .15$ \\
\hline 14 & 5 & 3 & 16 & 290288 & A & $169.169,165,156,155,153,151.147,142.137,133,124.109 .75,16,1$ \\
\hline 14 & 5 & 4 & 15 & 289872 & A & $169,169,163,156,155,152.149,148,142,1,39,1,32,131,102.76,19$ \\
\hline 14 & 6 & 1 & 14 & 680081 & $\mathrm{~B}$ & 253.252.243.24.3.24.3.24.3.212.212.212.212.212.212.212.42 \\
\hline 14 & 7 & 1 & 14 & 913176 & $\mathrm{~B}$ & 282.282 .280 .280 .271 .271 .271 .271 .271 .271 .271 .271 .70 .70 \\
\hline 14 & 7 & 2 & 15 & 887552 & B & $292.292 .280 .280 .272 .272 .2+2.2+2.2+2.2+2.242 .242 .242 .4 \times .2$ \\
\hline
\end{tabular}


impossible, for it would shorten to either $\pi(6,3)=$ $(4,4,4,4,4)$ or $(4,4,4,4,3,1)$. (Any class of size 6 must shorten in thrce ways to a class of size 4 and in four ways to a class of size 3 .) Therefore $(7,7,6,6,5,4)$ is optimal. This also implies the optimality of

$$
\boldsymbol{\pi}(8,4)=(14,14,12,12,10,8) .
$$

Second, we point out that there is no optimal partition $\pi(10,4)$. For $\pi_{1}(10,4)=(30,30,30,30,30,22,22,12,2,2)$ is maximal, as we now show, while $\pi_{2}(10,4)=(30,30,30$, $30,26,25,22,15,2)$ has fewer classes. Since $A(10,4,4)=30$, by Theorem 7 , no class has size greater than 30 . From [115] — see Table IV - the maximal number of classes of size 30 is five, this can occur in an essentially unique way. We use the particular set of five given in Table IV. Whon these are removed the remaining $\left(\begin{array}{c}10 \\ 4\end{array}\right)-5 \times 30=60$ vectors consist of

$\begin{array}{lll}(01111) & (00000) & (5) \\ (00000) & (01111) & (5) \\ ((0)(011) \times(00011) & (25) \\ (00101) \times(00101) & (25) .\end{array}$

Each of the first five vectors (and each of the second five) must be a different color. Among the final 50 vectors there cannot be a color class of size 21 , bccause if so then that class would contain at least three words of form $* * * * *(00011)$ or three of form $* * * * *(00101)$. Thus $\pi_{1}(10,4)$ is maximal.

Concluding remarks:

1) If $n=2 t, w=t, t$ odd, we may take $\epsilon=0$ and obtain

$$
A(2 t, 4, t) \geq \sum_{w-0}^{2^{-}} \operatorname{norm} \operatorname{II}(t, w) .
$$

Similarly if $t$ is even we get

$$
\begin{aligned}
A(2 t, 4, t) \geq \max \left\{\sum_{w-0.2, \cdot, t} \operatorname{norm} \Pi(t, w),\right. & \ln \operatorname{norm} \Pi(t, w)\} .
\end{aligned}
$$

2) Using their Construction B, Etzion and Van Pul [68] show that if $n$ is of the form $2^{k}(k \geq 2)$ or $3 \cdot 2^{k}(k \geq 2)$ and $w$ is even then Theorem 14 can be replaced by

$$
A(n, 4, w) \geq \frac{1}{n-1}\left(\begin{array}{c}
n \\
w
\end{array}\right) \text {. }
$$

From the partitions in Table VI (especially $\Pi_{2}(10,4)$ ) this now also holds for $n=5 \cdot 2^{k}(k \geq 2)$.

3 ) Romanov's construction [155] showing that $A(16,3)$ $\geq 2720$ (see Table II) also uses partitioning. We write the codewords in the form $(a, b)$, where length $(a)=9$, length $(b)=7$. On the left side $a$ has weight $0,3,6$, or 9 , and we make use of Kirkman's partition

$$
\pi(9,3)=(12,12,12,12,12,12,12)
$$

of the set of triples on 9 points into 7 disjoint copies $\mathscr{F}_{1}, \cdots, \mathscr{F}_{7}$ of $S(2,3,9)$ (see Tables IV, VI). On the right we partition the set of all 128 7-bit words into eight disjoint translates $\mathscr{H}_{0}, \mathscr{H}_{1}, \cdots, \mathscr{H}_{7}$ of the $[7,4,3]$ Hamming code. Romanov's code then consists of the vectors $\left(0, \mathscr{H}_{i}\right), \mathscr{H}_{i} \times \mathscr{H}_{i}(1 \leq i \leq 7)$, and thcir complements.

\section{Lower Beunds Obtainfi) by Modifying Comes with a Larger Minimal Distance}

The following inequalities, due to Zinovicv [187], Van Pul [149], [150], and Honkala et al. [94], resemble those of Section $\mathrm{V}$ in requiring very little computation. They produce good lower bounds for codes with $d=6$. We follow the treatment of Honkala et al. [94].

Theorem 20 ([187], [149], [150], [94]):

a) For $0 \leq g<\min \{w, \delta\}$ and $0 \leq k<n$ we have

$$
\begin{aligned}
A(n-k, 2 \delta-2 g, w-g) & \\
& \geq \frac{1}{\left(\begin{array}{l}
n \\
k
\end{array}\right)} A(n, 2 \delta, w) \sum_{i=0}^{g}\left(\begin{array}{c}
w \\
i
\end{array}\right)\left(\begin{array}{c}
n-w \\
k-i
\end{array}\right) .
\end{aligned}
$$

b) For $0 \leq g \leq w, 0 \leq k<n$ and $k-g<\delta$, wc have

$$
\begin{aligned}
A(n-k, 2 \delta-2 k+ & 2 g, w-g) \\
& \geq \frac{1}{\left(\begin{array}{c}
n \\
k
\end{array}\right)} A(n, 2 \delta, w) \sum_{i=g}^{k}\left(\begin{array}{c}
w \\
i
\end{array}\right)\left(\begin{array}{c}
n-w \\
k-i
\end{array}\right) .
\end{aligned}
$$

Proof: Suppose $P$ attains the bound $A(n, 2 \delta, w)$. For any $k$-subset $S$ of thc coordinates let $c_{S}$ denote the projection of $c \in \mathscr{C}$ into $S$, and let $c_{\bar{S}}$ denote the projection onto the other coordinates. A new codc $b_{S}$ with length $n^{\prime}=n-k, \quad d^{\prime}=2 \delta-2 g$ and $w^{\prime}=w-g$ is obtained by taking all words $c_{\bar{s}}$ for which $c \in \mathcal{f}$ and $w t\left(c_{S}\right)$ $=i$ for some $0 \leq i \leq g$, and complementing any $g-i 1$ 's. A counting argument shows that

$$
\sum_{|S|=k}\left|b_{S}\right|=A(n, 2 \delta, w) \sum_{i=0}^{g}\left(\begin{array}{c}
w \\
i
\end{array}\right)\left(\begin{array}{c}
n-w \\
k-i
\end{array}\right),
$$

and a) follows. To prove b) we take all words $c_{\bar{S}}$ for which $c \in f$ and $w t\left(c_{S}\right)=i$ for some $g \leq i \leq k$, and complement any $i-g 0$ 's.

The lower bounds on $A(17,6,7)$ and $A(18,6,7)$ in Table I-B are obtained from Theorcm 20a by taking $g=1$ and $f$ to be the Steiner system $S(5,8,24)$. The lower bounds on $A(15,6,7), A(16,6,7)$ follow similarly using a particular choice of $S$.

\section{Theorem 21 (Honkala et al. [94]):

$$
A(n-2, d-2, w-1) \geq A(n, d, w) \text {. }
$$

Proof: We modify the codewords $c$ for a code $b$ attaining $A(n, d, w)$ as follows. If $c$ ends with 00 , complement the final 1 , while if $c$ ends with 11 , complement the final 0 . Now omit the last two coordinates of all words.

The lower bounds $A(22,6,7) \geq 759, A(22,6,11) \geq 2576 \ddagger$ follow by taking $\mathscr{b}$ to be $S(5,8,24)$ or the code attaining $A(24,8,12)=2576$.

H. Hämäläinen [80] used a modification of this argument to show $A(21,6,6) \geq 269$. Start with the $S(5,8,24)$ 
TABLE VII

Sim-Constrained Lexicodes

\begin{tabular}{lr}
\hline \multicolumn{1}{c}{ Bound } & \multicolumn{1}{c}{$s$} \\
\hline$A(12,4,6)=132$ & 21 \\
$A(24,8,18) \geq 78$ & 175 \\
$A(25,8,18) \geq 254$ & 175 \\
$A(26,8,18) \geq 760$ & 175 \\
$A(26,8,19) \geq 256 \ddagger$ & 188 \\
$A(19,10,11)=12$ & 84 \\
$A(23,10,17)=8$ & 181 \\
$A(24,10,18)=9$ & 203 \\
$A(28,10,14) \geq 415 \ddagger$ & 130 \\
\hline
\end{tabular}

lexicodc (see the following section), and take thc $759-$ $330=429$ words that do not end with 00 . If such a word ends with 11 complement the final 0 . By omitting the last two coordinates we obtain 429 words of length 22 , distance 6 and wcight 7 . Using this set as the seed for a lexicographic code (scc the following section) we get another code showing $A(22,6,7) \geq 759$, in which (labeling the coordinates 1 to 22 from right to left) 269 words have a 1 in coordinatc 2 . Hence $A(21,6,6) \geq 269$.

\section{LeXicographic CODES}

Lexicographic codes are studied in detail in [44], and we refer to that paper for the general theory. Here we just consider constant weight lexicodes, which give easily computed lower bounds on $A(n, d, w)$ that are often reasonably good and in some cases give the best bounds known.

The constant weight lexicographic code (or lexicode, for short) with length $n$, Hamming distance $d$ and wcight $w$ is obtained by starting with the empty code, considering all binary vectors of the given length and weight in lexicographic order (beginning with $00 \cdots 011 \cdots 1$ ), and adding them to the code if they have the desired Hamming distance from it.

This is a "no-input" construction. The most remarkable example of a lexicode is the Steiner system $S(5,8,24)$ (see [44], [45]); other examples are indicated by " $x$ " in Table I.

Several variations are possiblc. The vectors of complementary weight $n-w$ may be used instead, or the vectors may be considered in Gray code order, or both. For example $A(25,12,9)=25$ and $A(27,12,18)=39$ also arise as Gray lexicodes.

Another modification, a sum-constrained lexicode, only considers binary vectors $\left(a_{0}, a_{1}, \cdots, a_{n-1}\right)$ that satisfy the constraint

$$
\sum_{i=0}^{n-1} i a_{i} \geq s,
$$

where $s$ is specified in advance. For example the choice $s=21$ produces thc Steiner system $S(5,6,12)$ ([44], [45]). Other examples are given in Table VII. Although a considcrable amount of computing is needcd to discover the best value of $s$, once found this gives a succinct definition of the code.

A more powerful modification is to start with an initial
TABLE VIII

LEXIC)(UDES WITH a SEED

\begin{tabular}{ll}
\hline \multicolumn{1}{c}{ Bound } & \multicolumn{1}{c}{ Seed } \\
$A(18,6,4)=22$ & 1422,24410 \\
$A(26,6,10) \geq 8189 \ddagger$ & $A(25,6,9) \geq 4100 \ddagger$ from Table XV \\
$A(26,6,17) \geq 5407 \ddagger$ & $A(25,6,16) \geq 4100 \ddagger$ from Table XV \\
$A(27,6,9) \geq 7198 \ddagger$ & $A(25,6,9) \geq 4100 \ddagger$ from Table XV \\
$A(27,6,10) \geq 11656 \ddagger$ & $A(25,6,10) \geq 5700 \ddagger$ from Table XV \\
$A(28,6,9) \geq 9577 \ddagger$ & $A(25,6,9) \geq 4100 \ddagger$ frøm Tablc XV \\
$A(26,8,13) \geq 3004 \ddagger$ & The lexicode $A(24,8,12)=2576$ \\
$A(27,8,13) \geq 3601 \ddagger$ & The lexicode $A(24,8,12)=2576$ \\
$A(21,10,7)=13$ & 310CC, 54524 \\
$A(23,12,10)=16$ & 58AA1C, $00 \mathrm{E} 4 \mathrm{~A} 6$ \\
\hline
\end{tabular}

set of vectors (the "seed") instead of thc cmpty set. Some codcs (labeled " $x y$ " in Table I) found this way arc best described in the condensed notation introduced in Section XII, and are listed in Tablc XVI. Others (indicated by " $x h$ " in Table I) are given in Table VIII. In the latter table, if thc seed has shorter word length than the final code, we pad the seed by adding prefixes $0 \cdots 01 \cdots 1$ of the appropriate weight on the left.

\section{iX. Constant Weight Codes from Transi.aies of Linear Codes}

A number of good constant weight codes may be obtained from translates of linear codes (and from translates of the Nordstrom-Robinson code, which behaves in many ways like a linear code). If $\mathscr{b}$ is an $[n, k, d]$ binary linear code, let $B^{(w)}(u)$ be the set of vectors of weight $w$ in the translate $u+\mathscr{b}, u \in \mathbb{F}_{2}^{n}$. Then

$$
A(n, d, w) \geq \max _{u \in \mathbb{F}_{2}^{\prime}}\left|B^{(w)}(u)\right| .
$$

Furthermore (cf. [28]) the code

shows that

$$
\left(B^{(w-1)}(u), 1\right) \cup\left(B^{(w)}(u), 0\right)
$$

$$
A(n+1, d, w) \geq \max _{u \in \mathbb{F}_{2}^{n}}\left\{\left|B^{(w-1)}(u)\right|+\left|B^{(w)}(u)\right|\right\} .
$$

A not very systematic search through known linear codes has yielded the following cxamples. The 14 vectors

$$
(1000000000000())(11101011100000)
$$

span Karlin's [28, 10, 8] self-dual codc ([132], p. 509, column 2 , first code). We apply (49) to the [27, 10, 7] code $\mathfrak{b}$ obtained by delcting the last coordinate. With $u=0$ we obtain $A(28,8,13) \geq 4668 \ddagger, A(28,8,14) \geq 5280$, with $u=$ $1 F$ we obtain $A(28,8,10) \geq 1652 \ddagger$, with $u=A$ we obtain $A(28,8,11) \geq 2666 \ddagger$, and with $u=15$ we obtain $A(28,8,12) \geq 3780 \ddagger$.

The generator matrix

\begin{tabular}{|l|l|l|l|l|l|}
\hline 011 & 011 & 011 & 011 & 011 & 001111111111 \\
011 & 101 & 101 & 101 & 101 & 110011111111 \\
101 & 011 & 110 & 101 & 110 & 111100111111 \\
101 & 110 & 011 & 110 & 101 & 111111001111 \\
110 & 110 & 101 & 011 & 110 & 111111110011 \\
110 & 101 & 110 & 110 & 011 & 111111111100 \\
\hline 110 & 110 & 110 & 101 & 101 & 010101010101 \\
\hline
\end{tabular}


TABLE IX

Weight Distributi@ns of Translates • F $[23,12,7]$ Golay Cede

\begin{tabular}{rrrrrrrrrrrrr}
\hline$\# \backslash i$ & 0 & 1 & 2 & 3 & 4 & 5 & 6 & 7 & 8 & 9 & 10 & 11 \\
\hline 1 & 1 & 0 & 0 & 0 & 0 & 0 & 0 & 253 & 506 & 0 & 0 & 1288 \\
23 & 0 & 1 & 0 & 0 & 0 & 0 & 77 & 176 & 176 & 330 & 616 & 672 \\
253 & 0 & 0 & 1 & 0 & 0 & 21 & 56 & 112 & 240 & 400 & 546 & 672 \\
1771 & 0 & 0 & 0 & 1 & 5 & 16 & 48 & 120 & 240 & 400 & 560 & 6.58 \\
\hline
\end{tabular}

defines a $[27,7,12]$ code (a less symmetrical code with thesc parameters is given in [87]). Again we apply (49) to the code $\ell$ formed by omitting the last coordinate. With $u=0$ we obtain $A(27,12,12) \geq 82$ and with $u=92120$ we get $A(27,12,13) \geq 81$.

Table IX gives the weight distributions of the cosets of the $[23,12,7]$ perfect Golay code ([132], Chap. 2). The first column gives the number of cosets with the given
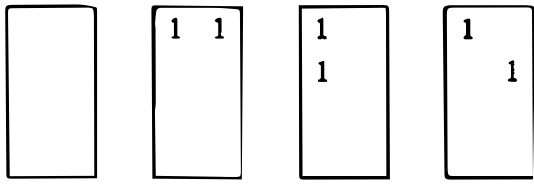

weight distribution. (In both Tables IX and $\mathrm{X}$ the weight distributions are symmetric about $n / 2$.) Using (49) we obtain $A(24,8,9) \geq 640$, as well as the other entries labeled " $t 4$ " in Table I.

The Nordstrom-Robinson code: The nonlinear Nordstrom-Robinson code of length 16, distance 6 and 256 codewords [132], [5], [107] produces a number of good constant weight codes, as was first observed by Semakov and Zinoviev [159]. We work inside the [24, 12, 8] extended Golay code $\mathscr{G}$ and represent codewords of $\mathscr{G}$ by $4 \times 6$ arrays called MOG's (or miracle octad generators). These have been described in several references (see [40], [42], [43], [46]-[48] and especially [45], pp. 303-304) and we do not repeat the definition here. We label the first 8 coordinatcs as follows (cf. [45], p. 316):

\begin{tabular}{|ll|l|l|}
\hline$\infty$ & 0 & & \\
3 & 2 & & \\
5 & 1 & & \\
6 & 4 & & \\
\hline
\end{tabular}

By deleting these 8 coordinates from the codewords of $\&$ we obtain (two copies of) the $[16,11,4]$ Hamming code $\mathscr{H}$, while the codewords of $\mathscr{G}$ that vanish on these 8 coordinates yield the $[16,5,8]$ first-order Reed-Muller code $\mathscr{R}$. Wc order the coordinates by reading down the columns, from left to right. When the Golay code defined by the MOG coordinates is read in this way it coincides with the lexicographic version of this code ([44], [45], p. 327).

Let $\mathscr{R}_{i}(0 \leq i \leq 6)$ denote the words of $\mathscr{G}$ that have 1 's in coordinates $\infty$ and $i$, and 0 's elsewhere in the first 8
TABLE $X$

Weight Distributiøns of Translates of Nordstrom-Robinsen Cone that Partition the Space

\begin{tabular}{|c|c|c|c|c|c|c|c|c|c|}
\hline$\# \backslash i$ & 0 & 1 & 2 & 3 & 4 & 5 & 6 & 7 & 8 \\
\hline 1 & 1 & 0 & 0 & 0 & 0 & 0 & 112 & 0 & 30 \\
\hline 16 & 0 & 1 & 0 & 0 & 0 & 42 & 0 & 85 & 0 \\
\hline 120 & 0 & 0 & 1 & 0 & 14 & 0 & 63 & 0 & 100 \\
\hline 112 & 0 & 0 & 0 & 5 & 0 & 33 & 0 & 90 & 0 \\
\hline 7 & 0 & 0 & 0 & 0 & 20 & 0 & 48 & 0 & 120 \\
\hline
\end{tabular}

coordinates, with the first 8 coordinates deleted. Each $\mathscr{R}_{i}$ is a translate of $\mathscr{R}$ containing 16 words of weight 6 and 16 of weight 10 , and

$$
\mathscr{N}=\mathscr{\mathscr { R }} \cup \mathscr{R}_{0} \cup \mathscr{R}_{1} \cup \cdots \cup \mathscr{R}_{6}
$$

is the Nordstrom-Robinson code. Thus $\mathscr{A}^{\prime}$ consists of the words of $\mathscr{G}$ that begin with one of
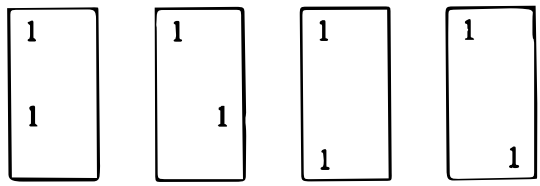

with thesc first 8 coordinates deleted.

Let $\alpha_{0}$ denote any of the four octads (weight 8 words in $\mathscr{G}$ ) that have 1 's at $\infty, 3,5,6$ and 0 's at $0,1,2,4$. Similarly $\alpha_{i}(0 \leq i \leq 6)$ is any octad that meets the first 8 coordinates just in $\infty$ and $3+i, 5+i, 6+i(\bmod 7)$. The seven translates $\alpha_{i}+. \mathcal{N}$ (with the first 8 coordinates deleted) together with $\mathscr{t}$ itself form a partition of the Hamming code $\mathscr{H}$. We remark that all seven translatcs $\alpha_{i}+\mathscr{N}$ are equivalent, all pairs of such translates are equivalent, and there are two inequivalent ways to choose three translatcs.

The Nordstrom-Robinson codc also has the property that certain of its translates partition the whole space of vectors of length 16 . The weight distributions of these translates are given in Table $\mathrm{X}$; thc last row describes the translates $\alpha_{i}+\mathscr{L}$. From Table $\mathrm{X}$ and (48) we obtain $A(16,6,6) \geq 112, A(16,6,8) \geq 120$.

The decomposition of $\mathscr{H}$ into 8 translates of $\mathscr{N}$ shows in particular that the 448 weight- 6 words in $\mathscr{H}$ can be partitioned as

$$
448=112+7 \times 48
$$

where each part has minimal distance 6 (see the $i=6$ column of Table $\mathrm{X}$ ). There is however a better partition of these 448 words. Let $\mathscr{f}_{1}$ denote the words of $\mathscr{G}$ that have exactly two 1's in thc first 8 coordinates, in coordinates $(\infty, 0),(\infty, 1),(\infty, 2), \cdots$, or $(\infty, 6)$, with the first 8 coordinates deleted. Similarly $f^{\prime}$ is obtained from the words of $\mathscr{G}$ that have 1 's in coordinates $(0,1),(0,2), \cdots$, $(0,6) ; \quad f_{3}$ from $(1,2),(1,3), \cdots,(1,6) ; \mathscr{f}_{4}$ from $(2,3),(2,4),(2,5),(2,6) ; \mathscr{\rho}_{5}$ from $(3,4),(3,5),(3,6) ;$ and $\rho_{6}$ from $(4,5),(4,6),(5,6)$. Each $f_{i}^{\prime}$ is a union of translates of $\mathscr{R}$. Let $\mathscr{f}_{i}^{(6)}$ denote the wcight 6 words in $\mathscr{f}_{i}$. Then $f_{1}^{(6)}, \cdots, \rho_{6}^{(6)}$ contain $112,96,80,64,48,48$ words, re- 
spectively. Since any two pairs defining an $\mathscr{f}_{i}^{\prime}$ have a point in common, and $\mathscr{G}$ has minimal distance 8 , it follows that each $\mathscr{f}_{i}^{(6)}$ is a constant weight code of minimal distance 6. All the $\rho_{i}^{(6)}$ are contained in $\mathscr{H}$, and so they partition the weight 6 words of $\mathscr{H}$ as

$$
448=112+96+80+64+48+48 .
$$

There is a similar result for wcight 5 words. Let $\mathscr{H}^{\prime}$ denote the Hamming code translated by $00 \cdots 01$. Then $\mathscr{H}^{\prime}$ is partitioned into 8 translates of $\mathscr{V}$ (one from the second row of Table $X$, seven from the penultimate row), which partitions the weight 5 words in $\mathscr{H}^{\prime}$ as

$$
273=42+7 \times 33
$$

(see the $i=5$ column of Table $\mathrm{X}$ ). An alternative partition

$$
273=42+36+4 \times 33+3 \times 21
$$

where each part has minimal distance 6 may be obtained as follows. We denote the nine parts by $\mathscr{Z}_{1}^{(5)}, \cdots, \mathscr{T}_{4}^{(5)}$, where $\mathscr{Z}_{i}^{(5)}$ consists of the weight 5 words in the translatc of $\Theta_{i}$ by $00 \cdots 01$ with thc first 8 coordinates deleted, and $\Theta_{1}=\mathscr{f}_{1}, \Theta_{2}=\mathscr{f}_{2}$. For $j=0,1,2,3, \Theta_{j+3}$ consists of the words of $\&$ having either two or four 1 's in the first cight coordinates, such that these 1's are a subset or superset of $\{1,3,4\}+j(\bmod 7) . \Theta_{7}$ consists of the words of $\mathscr{G}$ with two or four 1's in the first eight coordinatcs, such that these 1's are either the set $\{2,3\}$ or are a superset of $\{2,3, \infty\} . \Theta_{8}$ and $\Theta_{9}$ are defined in the same way as $\Theta_{7}$, replacing 2,3 by 4,6 and 1,5 respectively.

Adding tails to translates: The remaining codes in this section are found by adding tails to translates of the Nordstrom-Robinson and Golay codes. We denote by $B_{t}^{w}$ the set of vectors of weight $w$ in a translate of either of these codes by a vector of weight $t$. Thus $\left|B_{t}^{w}\right|$ is given by the entry in Table IX or $\mathrm{X}$ in the column headed $w$ and in the row in which the first nonzero entry occurs in column $t$.

The following codes in Table I arc obtained from the Nordstrom-Robinson code $\mathscr{N}$.

$A(20,6,6) \geq 232=112+6 \times 20$ from $B_{0}^{6} 0000,\left(B_{4}^{4}\right)^{4}\{1100\}$ $0 \leq i \leq 5$, where $\left(B_{4}^{4}\right)^{i}(0 \leq i \leq 5)$ represents the vectors of weight 4 in six translates of the type $\alpha_{i}+\ldots$ described in the last row of Table $X$, and $\{1100\}$ denotes all six binary 4-tuples of weight 2 .

$A(20,6,7) \geq 462=30+(112+96+80+64)+4 \times 20$ from $\mathscr{R}^{(7)^{\prime}} 00(0), \mathscr{f}_{i}^{(6)}(1000)(1 \leq i \leq 4)$ and $\left(\mathscr{H}_{4}^{4}\right)^{(j)}(0111)$ $\leq j \leq 3)$, where $\mathscr{R}^{(7)}$ is obtained by complementing the final 1 in each of the 30 weight -8 words in $\mathscr{R}$, and $f_{i}^{(6)}$ is defined above.

$A(20,6,8) \geq 588=120)+(112+96+80+64+48+48)+20$ from $\left(B_{4}^{8}\right)^{0} 0000, \mathcal{f}_{j}^{(6)}\{1100\},\left(B_{4}^{4}\right)^{0} 1111,1 \leq j \leq 6$.

$A(20,6,9) \geq 832=4 \times 120+(112+96+80+64)$ from $\left(B_{4}^{8}\right)^{i}(100()), \mathscr{f}_{j}^{(6)}(0111), 0 \leq i \leq 3,1 \leq j \leq 4$.

$A(20,6,10) \geq 944=112+6 \times 120+112$ from $B_{0}^{10} 0000,\left(B_{4}^{8}\right)^{l}\{1100\}, B_{0}^{6} 1111,0 \leq i \leq 5$.

$A(21,6,10) \geq 1382 \div=112+7 \times 120+30+(112+96+80+$ $64+48)$ from $B_{0}^{10} 00000,\left(B_{4}^{*}\right)^{i}\{11000\}, \quad 0 \leq i \leq 6$, $B_{0}^{r} 00011, \rho_{j}^{6}(01111), 1 \leq j \leq 5$.
$A(22,6,8) \geq 1116 \ddagger=6 \times 90+42+42+7 \times 33+7 \times 33+6 \times$ 5 from $\left(B_{3}^{7}\right)^{i}(100000), B_{1}^{5} 111000, B_{1}^{5}(000111$, $\left(B_{3}^{5}\right)^{j} t_{j},\left(B_{3}^{5}\right)^{j} \bar{t}_{j},\left(B_{3}^{3}\right)^{i}((011111), 0 \leq i \leq 5,0 \leq j \leq 6$, where $\left\{t_{0}, \cdots, t_{6}\right\}=\{110001,110010,11010(), 101001$, $101010,101100,011001\}$, and $\left(B_{3}^{7}\right)^{i}(0 \leq i \leq 5)$ represents the vectors of weight 7 in six cosets of the type described in the pcnultimate row of Table $X$, the cosets being chosen to have Hamming distance 4 apart. This code contains exactly 21 holes, all of which may be adjoined, yiclding $A(22,6,8) \geq 1137 \ddagger$. Further optimization by the methods described in Section XII gives $A(22,6,8) \geq 1139$ (see Table XVI).

$A(22,6,9) \geq 1736 \ddagger=6 \times 120+112+112+7 \times 48+7 \times 48+$ $6 \times 20$ from $\left.\left(B_{4}^{8}\right)^{i}(100000), B_{0}^{6} 11100\right), B_{0}^{6} 000111$, $\left(B_{4}^{6}\right)^{j} t_{j},\left(B_{4}^{(\mathrm{G}}\right)^{j} \bar{t}_{j},\left(B_{4}^{4}\right)^{i}(011111), 0 \leq i \leq 5,0 \leq j \leq 6$. This can be improved to $A(22,6,9) \geq 1768 \ddagger$ by first adding 28 holes in lexicographic order, then replacing the eight words FE3, 1DDA, 2A815A, 26419A, 30C523. $3300 \mathrm{DA}, 3304 \mathrm{~A} 3,3 \mathrm{C} 01 \mathrm{~A} 3$ by the twclve words 208F23, 210CE3, 2403E3, 24199A, 26605A. 28155A, 2AA09A, 300CDA, 30E063, 33211A, 332223, 3C2823. By shortening this code we obtain $A(21,6,9) \geq 1092 \ddagger$.

$A(22,6,10) \geq 2180 \ddagger=6 \times 90)+85+85+7 \times 90+7 \times 90+$ $(42+36+4 \times 33)$ from $\left.\left(B_{3}^{9}\right)^{i}(10000) 0\right), B_{1}^{7} 111000$, $B_{1}^{7} 000111,\left(B_{3}^{7}\right)^{j} t_{j},\left(B_{3}^{7}\right)^{j} \bar{t}_{j}, \mathscr{L}_{k}^{(5)}(111110), 0 \leq i \leq 5,0 \leq j$ $\leq 6,1 \leq k \leq 6$, where $\mathscr{Z}_{k}^{(5)}$ is defined above.

$A(22,6,11) \geq 2636=2 \times(448+30+7 \times 120)$ from $\mathscr{f}_{i}^{(h)}(011111)(1 \leq i \leq 6), B_{0}^{8} 111000,\left(B_{4}^{8}\right)^{\prime} t_{j}(0 \leq j \leq 7)$, and their complements.

$A(23,6,5) \geq 147=7 \times 20+7$ from $\left(B_{4}^{4}\right)^{t}(1000000)$, 000000000(1000000)(1110100), $0 \leq i \leq 6$.

The following codcs are similarly obtained from the $[23,12,7]$ Golay code:

$$
\begin{array}{ll}
A(26,8,11) \geq 1858 \ddagger & \left(B_{3}^{8} 111, B_{3}^{y} 110, B_{3}^{10} 100, B_{3}^{11} 000\right), \\
A(27,8,11) \geq 2047 \ddagger & \left(B_{0}^{7} 1111, B_{0}^{8} 1110, B_{0}^{11} 0000\right), \\
A(27,8,12) \geq 3082 \ddagger & \left(B_{0}^{8} 1111, B_{0}^{11} 1000, B_{0}^{12}(0) 00\right) .
\end{array}
$$

The final set of codes in this section come from the $[24,12,8]$ Golay code $\mathscr{G}$. Now $B_{t}^{w 1}$ denotes the vectors of weight $w$ in a translate of $\mathscr{G}$ by a vector of weight $t$ (see [46], [132], p. 69).

$A(25,8,9) \geq 829$ is obtained from the vectors $\left(B_{0}^{8}\right)^{\prime} 1$, $\left(B_{0}^{12}\right)^{\prime} 0$, where $\left(B_{0}^{8}\right)^{\prime}$ consists of the $\left.759-210\right)=549$ words of weight 8 in $\mathscr{G}$ not ending 000 , and $\left(B_{0}^{12}\right)^{\prime}$ consists of the 280 words of weight 12 ending 111 with thesc three 1 's complementcd (Kaikkonen [1(15]).

$A(25,8,10) \geq 1232 \ddagger=960+272$ is obtained from a translate of $\&$ containing 360 ) words of wcight 8 (denoted by $B_{4}^{8}$ ) and 960 words of weight 10 (denoted by $B_{4}^{(1)}$ ). We first take the 960 words $B_{4}^{10} 0$. Any vector $u$, where $u$ is obtained by complementing any 0 in a vector of $B_{4}^{8}$, is at distance 8 from the initial 960 words; there are $360 \times 16=$ 5760 such vectors, and we must find a subset of them at Hamming distance 8 apart. Wc could take the 240 out of the 360) that have a 0 in a particular coordinate and complement that coordinate, obtaining $A(25,8,10) \geq$ 
$960+240=1200 \ddagger$. However we can do better. Consider the subset of the 360 words with at most one 1 in a particular set of three coordinates, and in these coordinates replace 000 by 100,100 by 110,010 by 011 and 001 by 101. Consider first a random set of 3 out of the 24 coordinates. The probability that a vector containing 81 's and 160 's has at most a single 1 in three coordinates is

$$
\frac{\left(\begin{array}{c}
16 \\
3
\end{array}\right)+\left(\begin{array}{l}
8 \\
1
\end{array}\right)\left(\begin{array}{c}
16 \\
2
\end{array}\right)}{\left(\begin{array}{c}
24 \\
3
\end{array}\right)}=0.7510 \cdots
$$

so at least $360 \times 0.7510 \cdots=270.36 \cdots$ (hence 271 ) words can bc added in this way. A particular choice of three coordinates gives 272 . Thus $A(25,8,10) \geq 960+272=$ $1232 \ddagger$. By computer search it was found that 288 words can be added, yielding $A(25,8,10) \geq 960+288=1248$.

$A(25,8,11) \geq 1662=1218+444$ is similarly obtained from a translate of $\&$ containing 640 words $\left(B_{3}^{9}\right)$ of weight 9 and 1218 words $\left(B_{3}^{11}\right)$ of weight 11 . We first take the 1218 words $B_{3}^{11} 0$, and look for a subset of the $640 \times$ $15=9600$ vectors $u 1$ that can be adjoined, wherc $u$ is obtained by complementing any 0 in a vector of $B_{3}^{y}$. If we take the 400 out of the 640 with a 0 in a particular coordinate we get $A(25,8,11) \geq 1218+400=1618 \neq$. Again we can do better by using a subset of the 640 that have at most a single 1 in a particular set of three coordinates. By averaging we find that at least 640$) \times 0.6917 \cdots=$ $442.68 \cdots$ (hence 443) words can be added in this way. A particular choice of three coordinates gives 444, so $A(25,8,11) \geq 1218+444=1662$.

$A(26,8,10) \geq 1519=759+76(1)$ is found by starting with the 759 words $B_{0}^{8} 11$, and looking for a subset of the vectors $u 00$ to adjoin, where $u$ is obtained by complementing any two 1's in a word of $B_{0}^{12}$. We take the $120+4 \times 160=760$ words of $B_{0}^{12}$ that havc at most a single 0 in a set of four coordinates (see Fig. 2.15 of [132]), and replace 1111 by 0011,0111 by 0001,1011 by 1000,1101 by 0100 and 1110 by 0010 .

$A(26,8,12) \geq 3026 \ddagger=2576+450$ is obtained in a similar way from $B_{0}^{12} 00$ and $B_{0}^{8} 11$. There are $130+4 \times 80=450$ words in $B_{0}^{8}$ with at most a single 1 in a set of four coordinates (see Fig. 2.14 of [132]), and we now apply the complementary transformation to the previous one (replacing 0000 by 1100 , etc.). This construction can be improved as follows. We use the lexicographic vcrsion of $\mathscr{G}$, so that the octad $11 \cdots 100 \cdots 0 \in \mathscr{G}$. There are 256 octads in of the form $\{1000\}\{1000\} y$ (with each 1 in any of four positions). For each of these we form the vector $(1100)(1100) y 11$, and adjoin these vectors to $B_{0}^{12} 00$. This extends by "minimal dcgree lexicography" (see Section XII) to give $A(26,8,12) \geq 3070$.

$A(26,8,13) \geq 3328=2576+752$ is found by starting with the 2576 words $B_{0}^{12} 01$. There are 35420 vectors of weight 13 at distancc 8 from this set; they have the form $u 10$, where $u$ is the union of three words of weight 8 in $\mathscr{G}$ all at mutual distance 8 (see [45], Fig. 10.1, [46]). By com- puter it was found that 752 of these vectors can be adjoined to the 2576 .

$A(27,8,12) \geq 3146 \neq=2576+210+3 \times 120$ is obtained from $B_{0}^{12} 000$ and $B_{0}^{8} 111$, modifying the vectors in $B_{0}^{8}$ that have at most a single 1 in a set of three coordinatcs.

\section{Cones from Permutation Groups}

The codes in this section arc unions of orbits under a nontrivial permutation group. Let $G$ be a permutation group permuting the symbols $\{1, \cdots, n\}$. The orbit of a vector $x=\left(x_{1}, \cdots, x_{n}\right)$ under $G$ is the set of all vectors $x^{g}=\left(x_{g(1)}, \cdots, x_{g(n)}\right), g \in G$.

We first discuss groups generated by a single permutation $\pi$.

If $\pi$ is a cycle of length $n$ (equal to the length of the code), the code is a cyclic code, indicated by " $c$ " in Table I. Orbit representatives are listed in Table XI.

If $\pi$ is a cycle of length $n-1$, the code is an extended cyclic code, or "cyclic with a fixed point," indicated by "ec" in Table I. Orbit representatives are listed in Table XII.

If the permutation consists of a number of cycles of equal length the code is quasi-cyclic (see Table XIII). If there are $i$ cycles of length $n / i$ the code is indicated by " $q$ " in 'Table I.

The remaining codes defined by a single permutation ("polycyclic" codes) are listed in Table XIV, and indicated by " $p c$ " in Table 1.

The final tablc in this section (Table XV) lists codes that are defined by a group ( $;$ (of order $g$ ) having morc than one generator. These group codes are indicated by " $g$ " in Table I.

The first column in Table $X V$ gives the parameters of the code and [in brackets] the abstract type of $G$. The notation $q: r$ indicates that $G$ is isomorphic to a group of permutations of $\mathbb{F}_{a}$ of the form $x \rightarrow a x+b$, where $a$ bclongs to the multiplicative subgroup of $\mathbb{F}_{q}^{*}$ of order $r$, and $b \in \mathbb{F}_{q}$. (The colon indicatcs a semidirect product as in [41].) $Q(16)$ is a generalized quaternion group ([97], p. 91$)$.

The final column of Table XV givcs orbit representatives for the code, written in hexadecimal and right justified, with the orbit size as superscript. For example the first orbit representative for $A(12,4,6)=132$ is $B E^{55}$, indicating that the vector

$$
\begin{array}{rrrrrrrrrrr}
11 & 10 & 9 & 8 & 7 & 6 & 5 & 4 & 3 & 2 & 1 \\
0 & 0 & 0 & 1 & 0 & 1 & 1 & 1 & 1 & 1 & 0
\end{array}
$$

defines an orbit of size 55. The coordinates are numbercd from right to left.

Of course the full automorphism group of a code constructed in this scction may be much larger than the group wc use to construct it. For example, the first code in Table XV has as automorphism group the Mathieu group $M_{12}$ of order 95040 . 
TABLF XI

Cyculc Codes

\begin{tabular}{|c|c|}
\hline Bound & Other Representatives (in Hexadecimal) \\
\hline$A(22,9) \geq 68$ & 0, C984F, 11BDB5, 284347, 3FFFFF (Ref. [105]) \\
\hline$A(25,10) \geq 151$ & 0, 33947, BC5D3, 1492D5, 23EEBF, 2D3ED3, 358D99 \\
\hline$A(8,4,3)=8$ & B \\
\hline$A(13,4,3)=26$ & 13,85 \\
\hline$A(18,4,4)=198$ & $17,63, \mathrm{D} 1,129,303,419,445,885, \mathrm{~A} 09,1089,1421$ \\
\hline$A(19,4,3)=57$ & $43,89,405$ \\
\hline$A(25,4,3)=100$ & D, $841,2201,8101$ \\
\hline$A(26,4,3)=104$ & D, 441, 4201, 8101 \\
\hline$A(11,6,5)=11$ & 97 \\
\hline$A(12,6,5)=12$ & 97 \\
\hline$A(13,6,4)=13$ & B1 \\
\hline$A(13,6,6)=26$ & $1 \mathrm{AB}, 279$ \\
\hline$A(14,6,4)=14$ & 53 \\
\hline$A(14,6,6)=42$ & BB, 4C7, 52D \\
\hline$A(15,6,4)=15$ & $8 B$ \\
\hline$A(19,6,5) \geq 76$ & A7, 1503, 420B, 8449 (Ref. $[108])$ \\
\hline$A(20,6,5) \geq 84$ & $3043,11111,14025,20017,40883$ (Ref. [108]) \\
\hline$A(21,6,5) \geq 105$ & $343,1017,21049,28083,40423$ (Rcf. [108]) \\
\hline$A(26,6,4)=52$ & $20 \mathrm{~B}, 10811$ \\
\hline$A(27,6,4)=54$ & 883,4025 \\
\hline$A(15,8,7)=15$ & 537 \\
\hline$A(16,8,7)=16$ & $112 \mathrm{~F}$ \\
\hline$A(17,8,8)=34$ & B9D, 2D 13 \\
\hline$A(21,8,5)=21$ & 985 \\
\hline$A(23,8,5)=23$ & $410 B$ \\
\hline$A(24,8,5)=24$ & $20 \mathrm{Bl}$ \\
\hline$A(26,8,6)=130$ & 68B, 20139, 49015, 81843, 110^11 \\
\hline$A(19,10,9)=19$ & 5793 \\
\hline$A(20,10,9) \geq 20$ & $1129 \mathrm{I}$ \\
\hline$A(21,10,8) \geq 21$ & $1112 \mathrm{~F}$ \\
\hline$A(22,10,11) \geq 46$ & 12E6F, 3ED19, 155555 \\
\hline$A(24,10,7) \geq 24$ & $12 \mathrm{E} 11$ \\
\hline$A(24,10,9) \geq 56$ & $13 \wedge 35,84537$, В0В0В \\
\hline$A(24,10,10) \geq 72$ & $5348 \mathrm{~F}, 85 \mathrm{DC} 9,88 \mathrm{CB} 7$ \\
\hline$A(24,10,12) \geq 96$ & 4BE2F, 519F7, 1A4EE5, 1 DAC99 \\
\hline$A(25,10,10) \geq 100$ & 7B621, 8591F, 9AA4D, 151867 \\
\hline$A(25,10,11) \geq 125$ & 1D4B7, 421:37, B63^5, D954D, 1CF223 \\
\hline$A(26,10,10) \geq 130$ & $7 \mathrm{~B} 20 \mathrm{~B}, 8165 \mathrm{~F}, 1 \mathrm{C} 7131,235499,654 \mathrm{~A} 49$ \\
\hline$A(27,10,9) \geq 111$ & 19535, 85^2D, 1518EI, 923245, 1249249 \\
\hline$A(27,10,12) \geq 252 \ddagger$ & $\begin{array}{l}\text { 5AE4F, 1322BF, 2A0EEB, 3A55C5, 43F } 195 \\
\text { 4668DB, 5AB943, 62DE51, 9493CD, 9C4E27 }\end{array}$ \\
\hline$A(23,12,11)=23$ & $299 \wedge \mathrm{F}$ \\
\hline$A(24,12,10)=24$ & DE245 \\
\hline$A(24,12,11) \geq 24$ & $\wedge 65 \mathrm{~F} 1$ \\
\hline$A(26,12,9) \geq 26$ & $289 \mathrm{CB}$ \\
\hline$A(27,12,11) \geq 54$ & $2 \mathrm{CC} 789,42 \mathrm{~F} \wedge 23$ \\
\hline$A(28,12,12) \geq 84$ & 11D5E3, 532679, A17A1B \\
\hline$A(28,14,12) \geq 28$ & $8 \mathrm{C} 97 \mathrm{C} 5$ \\
\hline$A(28,14,13) \geq 28$ & $\wedge 2993 \mathrm{~F}$ \\
\hline
\end{tabular}


TABLE XII

Extended Cyclic (ok "Cyclic With a Fixen Point") Codes

\begin{tabular}{|c|c|}
\hline$\overline{\text { Bound }}$ & Orbit Representatives \\
\hline$A(17,4,4) \geq 156$ & 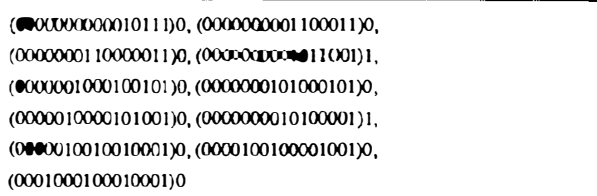 \\
\hline$A(17,8,7)=24$ & $(1110000100010110) 0,(1011000010110000) 1$ \\
\hline$A(22,10.10) \geq 42$ & $(100100100011110101000) 1,(101000111001101110000) 0$ \\
\hline$A(25,10,7) \geq 28$ & $(000000000100106010100111) 0,(000100010001000100010001) 1$ \\
\hline$A(25,10,8) \geq 48$ & $(100010001010100000110100) 0,(000000000110010010100011) 1$ \\
\hline$A(25,10,9) \geq 72$ & $\begin{array}{l}(00000101(x) 0111100101 \omega 1) 0,(000001000110010000110111) 0 \\
(\omega 0000011010011001000101) 1\end{array}$ \\
\hline$A(25,10,12) \geq 130$ & $\begin{array}{l}(00001010101101111100011) 0,(000001001110011101011011) 0, \\
(000010000111110110001011) 1,(000100101100111010101001) 1, \\
(000001111010100010001111) 1,(001001110010011100100111) 0, \\
(010101010101010101010101) 0\end{array}$ \\
\hline$A(27,10,11) \geq 208 \ddagger$ & $\begin{array}{l}(000010001101010101(0) 100101) 1,(00000011110011000010011011)), \\
(00010011001011010100010101) 0,(00000100111001000111000011) 1, \\
(00001000011010100100110111) 0,(000000110000111010011100111) 0, \\
(00000010001000001011011111) 1,(00000001001000111110101011) 0\end{array}$ \\
\hline$A(28,10,10) \geq 192 \ddagger$ & $\begin{array}{l}(000000100101000101110010001) 1,(000000010101001110100001101) 0 \\
(00000101100101001001010001) 0,(000000001000011110101110001) 0, \\
(000000010111000010001110001) 1,(000000001110010010011001101) 0, \\
(000001101011000100001010101) 0,(001001001001001001001001001) 1\end{array}$ \\
\hline$A(28,10,11) \geq 270 \ddagger$ & $\begin{array}{l}(000010001101000011100010111) 0,(000000000011011010001110011) 1, \\
(00001000100010101001111011) 0,(000100101010010100100010101) 1, \\
(000010010001100010111011001) 0,(00000110101100100110100101) 0, \\
(000001010100001000011010111) 1,(000010001110010000111110001) 0 . \\
(000000110000110111001001001) 1,(000000100101000010011101111) 0\end{array}$ \\
\hline$A(25,12,11) \geq 36$ & $(001101000000110010101111),(000100101110000100101110) 1$ \\
\hline$A(27,12,10) \geq 39$ & $(00100000101110010000010111) 0_{(}(\operatorname{cec} 000000101000100111001011) 1$ \\
\hline
\end{tabular}

A number of different computer programs were used to find the group-invariant codes described in this section. The following seems to be the most efficient method. Given a permutation group $G$, we first find its orbits on the 0 -element subset of the coordinates (there is only one!). Given representatives for the $i$-element subsets, we extend these in all possible ways by a singleton and find among the vectors thus obtained the (lexicographically minimal) represcntatives for the orbits on $(i+1)$-subsets. At the same time this tells us how often each type of $i$-subset occurs in an $(i+1)$-subset of given type. This process is continued until representatives for the $i$-elemont subsets with $i \leq w$ have been found.

For $t=w-d / 2+1$, we form a matrix $B$ indexed by orbits of $w$-sets and $t$-sets, specifying how often a $t$-set is covered by the vectors in a given orbit of $w$-sets. Orbits of $w$-sets for which the corresponding row of $B$ contains an entry greater than 1 can be discarded.

We now define a graph on the remaining $w$-set orbits, joining two of them when they do not cover the same $t$-set, i.e., when the corresponding rows are orthogonal. The largcst codes invariant under $G$ are obtained as the largest weighted cliques in this graph, where the weights are the orbit sizes. This method requires enough space to store the $T_{w} \times T_{t}(0,1)$-matrix $B$, where $T_{i}$ is the number of orbits on $i$-sets.
TABLE XIII

Quasi-C'yclic Cones

\begin{tabular}{|c|c|}
\hline Bound & Orbit Representatives \\
\hline$A(16,8,6)=16$ & $(00110101)(00011000),(00011000)(01010011)$ \\
\hline$A(18,8,6)=21$ & $\begin{array}{l}(110100)(100000)(110000),(000010)(110100)(100001) \\
(000011)(100001)(010100),(010101)(010101)(000000), \\
(000000)(00000)(11111)\end{array}$ \\
\hline$A(18,10,8)=9$ & $(000100111)(000101101)$ \\
\hline$A(20,10,7)=10$ & $(0000101011)(0000010011)$ \\
\hline$A(26,10,6)=13$ & $(0000000011001)(000001010001)$ \\
\hline$A(27,10,6)=14$ & $\begin{array}{l}(010)(000)(000)(01))(001)(010)(101)(000)(000) \\
(010)(000)(000)(100)(000)(100)(000)(101)(010) \\
(000)(010)(100)(000)(000)(001)(001)(000)(011) \\
(000)(001)(100)(000)(110)(010)(000)(001)((000)) \\
(111)(111)(000)(000)(000)(000)(000)(000)(000) \\
(000)(000)(111)(111)(000)(000)(000)(000)(000)(\text { Ref. }[105])\end{array}$ \\
\hline$A(27,10,7) \geq 36$ & $\begin{array}{l}(000010111)(00000100)(010000100) \\
(000100011)(001010000)(000111000) \\
(0000000001)(001000001)(100001110) \\
(000001001)(011100100)(000000001)\end{array}$ \\
\hline$A(28,10,7) \geq 37$ & 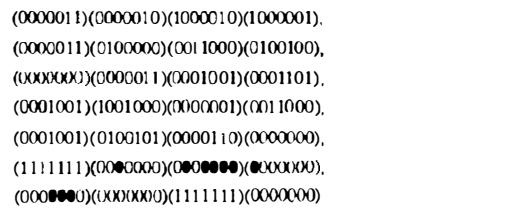 \\
\hline
\end{tabular}

$A(25,12,8)=10 \quad(110)(1))(111(K)(0)(10100)(10100)(00000),(00100)(00001)(11000)(00100)(11010)$

$A(26,12,11) \geq 39 \quad(0001001111011)(0001101010000),(0001110601100)(0011000101011)$ $(0011011001000)(000111100101)$

$A(27,12,8)=15 \quad(001)(000)(010)(000)(000)(001)(010)(101)(101)$ $(100)(010)(010)(001) ; 000)(110)(110)(000)(000)$ $(011)(000)(010)(001)(101)(000)(001)(000)(010)$. $((\mathbf{X}) 1)(011)(100)(101)(000)(000)(000)(010)(100)$ $(000)(011)(000)(000)(011)(001)(100)(1 \mathbf{6 0})(010)($ Ref. [105]

$A(26,14,12)=13 \quad(0001001001111)(0001001010111)$

$A(27,14,12) \geq 19 \quad(000)(000)(111)(111)(111)(000)(000)(000) 111$ $(110)(110)(100)(110)(100)(000)(100)(110) 100$. (011)(101)(100)(001)(110)(1 10)(000)(100)010, $(011)(110)(011)(100)(100)(100)(011)(000) 001$ $(100)(100)(000)(011)(100)(010)(011)(011) 011$. $(010)(100)(001)(000)(011)(110)(100)(011) 101$ $(010)(001)(101)(100)(000)(011)(011)(010) 110$

$A(28,14,11)=21 \quad(1000000)(0011101)(0110001)(1101000) .(1010001)(100000)(1001110)(1101000)$ $(0101110)(0100011)(1000000)(1101000)$

\section{Xi. Miscellaneous Constructions}

In this section we give some isolated constructions that do not fit into any other category.

The group code $b$ showing that $A(16,4,8) \geq 1164 \ddagger$ (see Table XV) leads to three other good codes.

$A(16,4,8) \geq 1170$. In $f$, replace the ten words $\mathrm{FF}$, AF5, 11EE, 24DB, 7D82, BE41, C03F, CF30, F30C, FF00 by the sixteen words $1 \mathrm{EF}, 2 \mathrm{~F} 7,4 \mathrm{DF}, 8 \mathrm{FD}, 10 \mathrm{FE}, 20 \mathrm{FB}$, 07F, 7F02, 80BF, BF01, DF10, EF20, F704, FB08, FD80, E40.

$A(14,4,6) \geq 276 \ddagger$. Shortcn $-b$ by taking the 275 words with a 1 in the first (i.e., left-most) and tenth coordinates, and adjoin 58B.

$A(14,4,7) \geq 317 \ddagger$. Shorten $\mathbb{C}$ by taking the 314 words beginning 10 , and adjoin $\mathrm{BF}, \mathrm{B} 4 \mathrm{~B}, 3 \mathrm{~F} 80$.

$A(22,6,4)=37$. Take a Kirkman triple system of order 15 ([14], [30]), i.e., a Steiner system $S(2,3,15)$ in which the 35 blocks are partitioned into seven "parallel classes" 
TABLE XIV

Other Codes Generated by a Single Permutation, and Related Codes

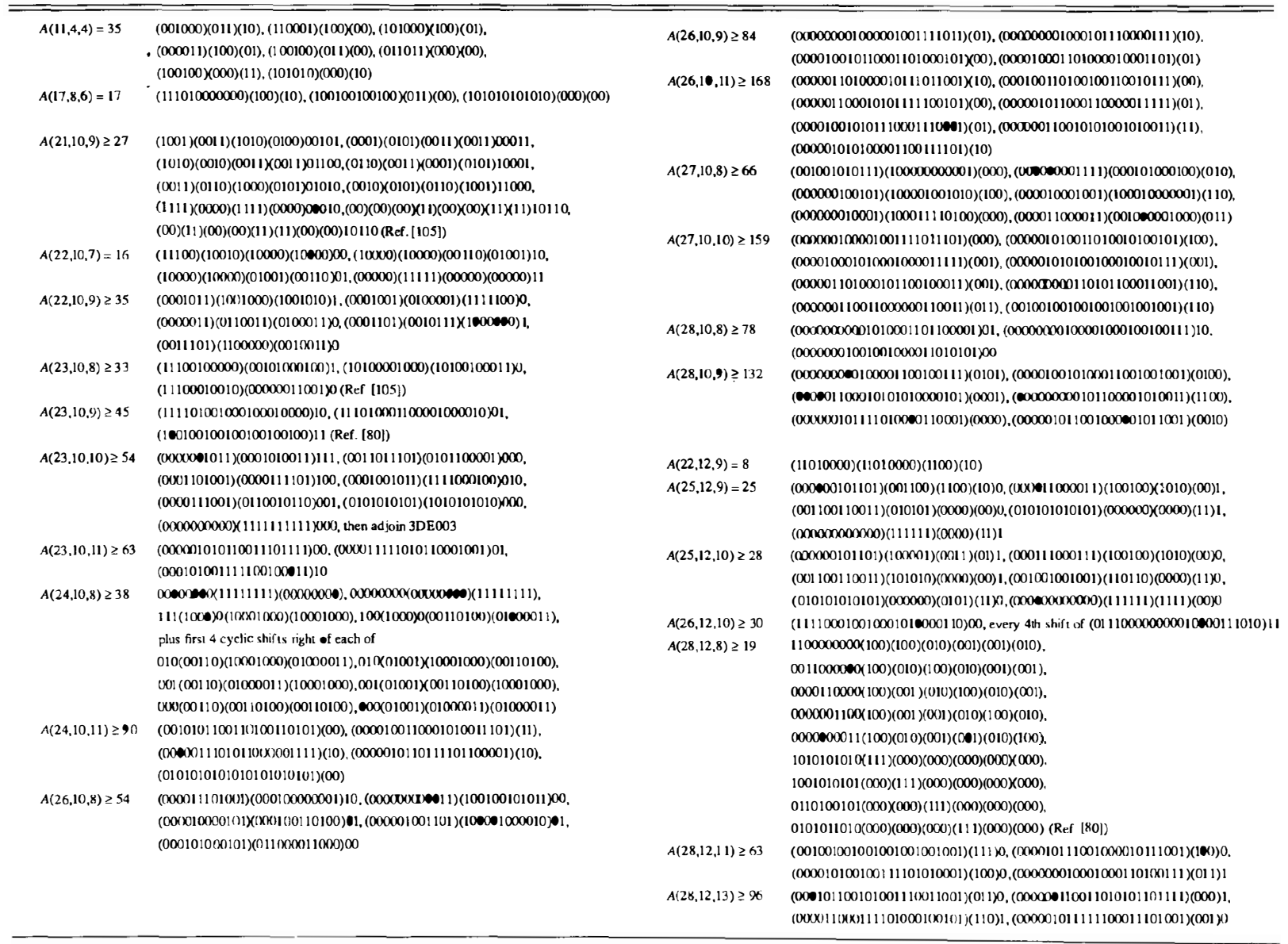

each containing tive disjoint blocks. Add one further point "at infinity" to each parallel class, yielding 35 words of length 22 and weight 4 , and adjoin $A(7,6,4)=2$ words on the 7 extra points.

$A(22,6,5) \geq 132$. In the weight 5 words of the $[11,6,5]$ ternary Golay code, replace 0 by 00,1 by 01 and 2 by 10 .

$A(28,8,5)=33$. Start with the affine planc $A G(2,5)$, containing 305 -sets (the lines) on 25 points, and adjoin three points $X_{1}, X_{2}, X_{3}$. Choose three noncollinear points $P_{1}, P_{2}, P_{3}$, and in the line $P_{1} P_{2}$ replace $P_{1}$ by $X_{3}$, in the line $P_{2} P_{3}$ replace $P_{2}$ by $X_{3}$, in the line $P_{3} P_{1}$ replace $P_{3}$ by $X_{3}$, and adjoin the 5-set $P_{1} P_{2} P_{3} X_{1} X_{2}$. Repeat this with three further noncollinear points $Q_{1}, Q_{2}, Q_{3}$ (replacing $Q_{1}, Q_{2}, Q_{3}$ in the three lines by $X_{2}$ and adjoining $Q_{1} Q_{2} Q_{3} X_{1} X_{3}$ ), and again with three noncollinear points $R_{1}, R_{2}, R_{3}$, making sure that $\left\{P_{1}, P_{2}, P_{3}, Q_{1}, Q_{2}, Q_{3}\right\}$, $\left\{P_{1}, P_{2}, P_{3}, R_{1}, R_{2}, R_{3}\right\}$ and $\left\{Q_{1}, Q_{2}, Q_{3}, R_{1}, R_{2}, R_{3}\right\}$ are conics in the affine plane. The final code is shown in Fig. 1.

$A(20,10,8)=17$ is constructed in Fig. 2 .

$A(28,10,6)=16$ follows by shortening the Steiner system $S(2,6,31)=P G(2,5)$.
Finally Kaikkonen [105] observed that if $n$ is even and $d^{\prime}=\min \{n, 2 d\}$ then

$$
A\left(2 n, d^{\prime}, n\right) \geq A(n, d)+A\left(n, d, \frac{n}{2}\right)
$$

This is obtained by replacing 0 by 01 and 1 by 10 in the code attaining $A(n, d)$, and 0 by 00 and 1 by 11 in the code attaining $A(n, d, n / 2)$. For examplc $A(28,12,14) \geq$ $A(14,6)+\Lambda(14,6,7)=106$. Many generalizations are possible, for cxample using ternary codes, but do not seem to lead to new records in the range of our tables.

\section{Siarching for Codes with a Computer; Codes with no Known Siructure}

In preparing Table I we made use of several computer programs that searched for codes. Two kinds of programs were used, exhaustive search methods and hcuristic (noncxhaustive) methods. In discussing running times, besides the usual variables $n, d, w$ and $M$ (the number of 
codewords), we use $U$ to denote $\left(\begin{array}{l}n \\ w\end{array}\right)$, the size of the universe of possible codewords.

Exhaustive search methods: We explain our exhaustive scarch technique by describing the proof that $A(14,6,7)$ $=42$. A code with 42 words was constructed in Scction III, so it suffices to show that no code exists with 43 words. In principle we must consider all possible subsets of 43 words from a universe of size $U=\left(\begin{array}{c}14 \\ 7\end{array}\right)=3432$.

However, the size of this search space may be greatly reduced. First, from (5), any code attaining $A(14,6,7)=43$ must contain a subcode $C^{\prime}$ with $n=13, d=6, w=7$, $M=22$, and $C^{\prime}$ must contain a subcode $C^{\prime \prime}$ with $n=12$, $d=6, w=7, M=11$. A previous exhaustive search has determined that there are precisely 95 incquivalent choices for $C^{\prime \prime}$. We may now restrict our search to codes that contain one of these codes as the first 11 words.

Next, we need not consider every subset of the weight-7 14-bit vectors as a possible code. Only scts of vectors with all pairwise distances $\geq 6$ need be considered. All such sets may be generated by a standard backtracking algorithm, indeed in lexicographic order.

The search spacc may be further reduced by noticing that any $M$-word code $C$ has $M ! n ! / \mid$ Aut $\left(C^{\prime}\right) \mid$ isomorphic versions (obtained by permuting the $n$ coordinates and the $M$ codewords). We wish our search to find exactly one (or at any rate very few) of the codes in each such equivalence class. The $\boldsymbol{M}$ ! factor is avoided by requiring that any code generated must be in lexicographic order-a condition readily incorporated into the backtracking algorithm. A large part of the $n$ ! factor is automatically removed by the fact that the first 11 words form one of the subcodes $C^{\prime \prime}$ previously mentioned, and the first 22 words form a subcode $C^{\prime}$.

More generally we may require all generated codcs to be in "canonical form": namely lcxicographically least under any permutation of coordinates and corresponding resorting of codewords, while preserving the property that the Johnson subcode $A(n-1, d, w)$ lies in the first $n-1$ coordinates and constitutes the first $[(n-w) M / n]$ words (and so on recursively for the subcodes of this code,...).

Proving that a code is canonical is difficult, but some simple tests can readily show that a code is not canonical. If any dcparture from canonical form does occur during the backtracking, we may immediately prune that branch of the search.

The combination of all of these ideas made this initially intractable search problem solvable (in less than $18 \mathrm{~min}$ utes on an IBM 3090 model S computer, including the time to generate the 95 incquivalent codes $C^{\prime \prime}$ ), using a program written in a combination of Fortran and IBM-370 assembly language. The search tree contained about 90 million nodes, 309704 of them being codes $C^{\prime}$ with $n=13$, $d=6, w=7, M=22$ (we did not attempt to sort these into equivalence classes). Other exact values of $A(n, d, w)$ found in this way are given in Table III.

Heuristic search methods: For problems too large to be attacked by exhaustive search, we must be content with heuristic algorithms. Perhaps the most straightforward hcuristic is simply to run the exhaustive search describcd above, or a variant of it, performing an incomplete search, and keep the best code found.

Often we are given a good partial code (obtained for example by shortening another code), and wish to complete it. Several heuristic methods are available. The lexicographically least code containing some given "seed" subcode is readily found in $O(U M)$ steps. A similar, but more powerful code-extension heuristic is "minimaldegree lexicography." Consider the $H$-vertex graph formed by the $H$ holes in a partial code (two verticcs being joined by an edge if the corresponding vectors differ in less than $d$ places). Remove the lexicographically least vcrtex of minimal degree from this graph (as well as all its neighbors), and place it in the codc; continue doing this (using the sequence of successively smaller graphs that arisc) until no further augmentation is possible. This procedure takes $O\left(U M+H^{2}\right)$ time.

A simple probabilistic variant of these two methods uses "coin tossing." Namely, each time the extension heuristic could add a new vector to the current code, we toss a (possibly biased) coin, and add the vector only if the coin toss comcs up "heads." Otherwise ("tails") we discard the vector and continue. Alternatively, deterministic skip-selection methods (such as a systematic backtrack search, or a greedy procedure) may be used.

Another kind of approximate optimization is based on "local search." We say that a code is " $k$-optimal" if its cardinality cannot be incrcascd (while maintaining the minimal distance) by deleting $k$ words and adding holes. Thus lexicodes (and other codes found by a greedy algorithm) are 0-optimal, while codes attaining $A(n, d, w)$ are $k$-optimal for all $k$. If $k$ is small then an $M$-word code $C$ may bc tested for $k$-optimality (and if not $k$-optimal an improvement found) in $O\left(U M+\left(\begin{array}{c}M-1 \\ k-1\end{array}\right) k T\right)$ time, where $T$ is the sizc of the following bipartite graph.

This graph, which is constructed at the beginning of the search, has two sets of verticcs, a blue vertex for every codeword of $C$ and a red vertex for every vector not in $C$ that "bites" (lies at distance less than $d$ from) $k$ or fewer words of $C$; an edge joins each such vector to the codewords that it "bites." Oncc this graph is constructed, no further holc-finding need be done.

We now consider all possible $k$-subsets $S$ of $C$ (the blue vertices), and see if their dcletion will increase the size of $C$, using a trivial exhaustive search among the red vertices that are connected only to $S$.

The following "polishing" proccdure was of ten able to improve even thesc $k$-optimal codes. We begin by constructing the bipartite graph described above, maintaining the red vertices (those not in the code) in a queue. If there is a red vertex $h$ of degree 1 we exchange $h$ with the codeword $c$ to which it is connected, adding $h$ to the code in place of $c$, which is placed at the end of the queue. When there are several choices for $h$ we choose the one closest to the front of the queue, i.e., which has been out of the code longest. This procedure is then repeated. If after some fixed number (c.g., 1000) of itera- 
TABLE XV

Codes Define\ by Groups with muke rHan @ne Generator, and Related Codes

\begin{tabular}{|c|c|c|c|}
\hline $\begin{array}{l}\text { Bound } \\
\text { [Group] }\end{array}$ & $g$ & Generating Permutations & Orbit Representatives \\
\hline $\begin{array}{l}A(12,4,6)=132 \\
{[11: 5]}\end{array}$ & 55 & $(1,2,3, \ldots, 11),(2,5,6,10,4)(3,9,11,8,7)$ & $5 \mathrm{~F}^{55}, 83 \mathrm{D}^{55}, \mathrm{ED}^{11}, 897^{11}$ \\
\hline $\begin{array}{l}A(14,4,4)=91 \\
{[7: 6]}\end{array}$ & 42 & $\begin{array}{l}(1,2,3,4,5,6,7)(8,9,10,11,12,13,14) \\
(1,14)(2,12,5,13,3,10)(4,8,6,11,7,9)\end{array}$ & $\begin{array}{l}186^{21}, 198^{21}, 1 \mathrm{E0^{21 }} \\
17^{14}, \mathrm{D}^{14}\end{array}$ \\
\hline $\begin{array}{l}A(14,4,5) \geq 169 \\
{[13: 12]}\end{array}$ & 156 & $(1,2,3, \ldots, 13),(1,2,4,8,3,6,12,11,9,5,10,7)$ & $5 D^{73}, 2053^{52}, 6 B^{39}$ \\
\hline $\begin{array}{l}A(14,4,7) \geq 316 \ddagger \\
{[7: 6]}\end{array}$ & 42 & $\begin{array}{l}(1,2,3,4,5,6,7)(8,9,10,11,12,13,14) \\
(1,3,2,6,4,5)(8,10,9,13,11,12)\end{array}$ & $\begin{array}{l}19 \mathrm{~F}^{42}, 3 \mathrm{~B}^{42}, 5 \mathrm{D} 5^{42}, 793^{42} \\
7 \mathrm{AS}^{42}, \mathrm{BAC}^{42}, \mathrm{FBO}^{12}, 5 \mathrm{CB}^{14} \\
\mathrm{FE}^{7}, 3 \mathrm{~F} 80^{1}\end{array}$ \\
\hline$A(15,4,5) \geq 234 \ddagger$ & 42 & $\begin{array}{l}\text { same group as above } \\
\text { then adjoin vectors } 1 \mathrm{~F}, 67,79\end{array}$ & $\begin{array}{l}19 \Lambda^{42}, 1 \wedge 6^{42}, 418 \mathrm{C}^{42}, 1 \mathrm{D} 4^{21} \\
385^{21}, 7 \mathrm{~A} 0^{21}, 4099^{21}, 4382^{21}\end{array}$ \\
\hline$A(15,4,6) \geq 382 \ddagger$ & 42 & $\begin{array}{l}\text { then adjoin } 31 \text {; }, F A 0,1790,1 \mathrm{BB88}, 1 \mathrm{D} 84,1 \mathrm{E} 82 \text {, } \\
11: 40,2 \mathrm{~F} 01,6780,7980,7 \mathrm{E} 00\end{array}$ & $\begin{array}{l}18 \mathrm{~F}^{42}, 399^{42}, 3 \Lambda \Lambda^{42}, 5 C 6^{42}, 783^{42} \\
4394^{42}, 4585^{42}, 40 B C^{21}, 4193^{21} \\
43 \mathrm{C}^{21}, 5 B 4^{14}\end{array}$ \\
\hline $\begin{array}{l}A(16,4,6) \geq 592 \ddagger \\
{\left[2^{4}: 5\right]}\end{array}$ & 80 & $\begin{array}{l}(1,15,7,5,12)(2,9,13,14,8)(3,6,10,11,4) \\
(1,16)(2,3)(4,5)(6,7)(8,9)(10,11)(12,13)(14,15)\end{array}$ & $\begin{array}{l}\mathrm{BB}^{80}, 1 \mathrm{~F}^{80}, 2 \mathrm{BC}^{80}, 297^{80} \\
30 \mathrm{~F}^{80}, 94 \mathrm{E}^{80}, 6 \mathrm{~F}^{40}, 378^{40} \\
2 \mathrm{CE}^{16}, 365^{16}\end{array}$ \\
\hline $\begin{array}{l}A(16,4,8) \geq 1164 \ddagger \\
{[P S L(2,7)]}\end{array}$ & 168 & $\begin{array}{l}(1,2,3,4,5,6,7)(9,10,11,12,13,14,15) \\
(2,3,5)(4,7,6)(10,11,13)(12,15,14) \\
(1,8)(2,7)(3,4)(5,6)(9,16)(10,15)(11,12)(13,14)\end{array}$ & $\begin{array}{l}75 \mathrm{D}^{168}, 76 \mathrm{~B}^{168}, \mathrm{~F} 78^{168}, 1 \mathrm{~F} 23^{168} \\
1 \mathrm{~F}^{16 \mathrm{C}^{168}}, 173 \mathrm{~A}^{84}, 737^{56}, 1 \mathrm{~F} 15^{56} \\
\mathrm{FOF}^{42}, 3 \mathrm{FC}^{28}, 3 \mathrm{FC} 0^{28}, 17 \mathrm{E} 8^{14} \\
1 \mathrm{DE} 2^{14}, \mathrm{FFOO} 0^{1}, \mathrm{FF}^{1}\end{array}$ \\
\hline $\begin{array}{l}A(18,4,5) \geq 516 \ddagger \\
{\left[3^{2}: 8\right]}\end{array}$ & 72 & $\begin{array}{l}(1,5,9)(2,3,8)(4,7,6)(10,14,18)(11,12,17)(13,16,15) \\
(1,2,3,4,5,6,7,8)(10,11,12,13,14,15,16,17) \\
\text { then adjoin } 643, C 86,1 \text { A0C., 3418, 6814, C068, } \\
14281,1 \mathrm{~A} 090,22111,24422,28844,31088\end{array}$ & $\begin{array}{l}21 \mathrm{~B}^{72}, 64 \mathrm{C}^{72}, 6 \wedge 4^{72}, \mathrm{E} 14^{72} \\
\mathrm{E} 81^{12}, \mathrm{~F} 02^{72}, 1 \mathrm{E} 40^{72}\end{array}$ \\
\hline $\begin{array}{l}A(20,4,6) \geq 2280 \\
{[P S L(2,19)]}\end{array}$ & 3420 & $\begin{array}{l}(1,2,3, \ldots, 19) \\
(2,5,17,8,10,18,12,7,6)(3,9,14,15,19,16,4,13,11) \\
(1,20)(2,19)(3,10)(4,7)(5,15)(6,16)(8,9)(11,18)(12,13)(14,17)\end{array}$ & $5 \mathrm{~F}^{1710}, \mathrm{~F}^{570}$ \\
\hline$A(20,4,10) \geq 13452$ & 3420 & same group as ahove & 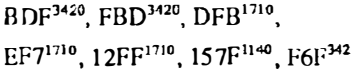 \\
\hline
\end{tabular}

tions the code has not bccn improved, the vector at the front of the queue is added to the code and its neighbors are removed and placed at the end of the qucue (temporarily decreasing thc size of the code).

In the range of our tables we are able to achieve $k$-optimality for values of $k$ ranging $2-5$. A less conservative attack would allow $k$-alterations for much larger values of $k$, considering only a small fraction of possible $k$-scts, without trying to achievc $k$-optimality. One such heuristic code-improver is the following.

1) Perform a permutation on the coordinates of the code.
2) Perturb every codeword by "pushing" it until it is lexicographically as small as possible.

3) Sort the (permuted and pushed) codewords into lexicographic order.

4) Remove the (lexicographically) last $k$ words from the code, and attempt to replace them by more than $k$ words, using somc exhaustive or heuristic search method.

5) Go back to Step 1) (and repeat as many times as desired).

In this procedure onc can use very large values of $k$, e.g., $20 \%$ of the codewords. This is one of a class of possible 
TABLE XV (Continued)

\begin{tabular}{|c|c|c|c|}
\hline $\begin{array}{l}\text { Bound } \\
\text { [Group] }\end{array}$ & $g$ & Generating Permutations & Orbit Reprcsentatives \\
\hline $\begin{array}{l}A(24,4,6)=7084 \\
{[P S L(2,23)]}\end{array}$ & 6072 & $\begin{array}{l}(1,2,3, \ldots, 23) \\
(2,3,5,9,17,10,19,14,4,7,13)(6,11,21,18,12,23,22,20,16,8,15) \\
(1,24)(2,23)(3,12)(4,16)(5,18)(6,10)(7,20)(8,14) \\
\quad .(9,21)(11,17)(13,22)(15,19)\end{array}$ & $6 \mathrm{~F}^{3036}, 1 C \mathrm{D}^{3036}, 197^{1012}$ \\
\hline$A(24,4,8) \geq 34914$ & 6072 & same group as above & $\begin{array}{l}5 B D^{6072}, 67 D^{3036}, 72 F^{3036} \\
9 D B^{3036}, \mathrm{ADD}^{3036},{\mathrm{~B} 67^{3036}} \\
E E 5^{3036}, \mathrm{~F} 39^{3036}, \mathrm{FA}^{3036} \\
12 \mathrm{BD}^{3036}, 149 \mathrm{~F}^{759}, 351 \mathrm{~B}^{759}\end{array}$ \\
\hline $\begin{array}{l}A(28,4,6) \geq 15288 \\
{[\operatorname{PSL}(2,13)]}\end{array}$ & 1092 & $\begin{array}{l}(1,2,5,3,10,6,12,4,9,11,8,7,13) \\
\quad(15,16,19,17,24,20,26,18,23,25,22,21,27) \\
(1,3,5,7,9,11)(2,4,6,8,10,12)(15,17,19,21,23,25) \\
\quad(16,18,20,22,24,26) \\
(1,7)(2,6)(3,5)(8,12)(9,11)(13,14)(15,21)(16,20) \\
\quad(17,19)(22,26)(23,25)(27,28)\end{array}$ & 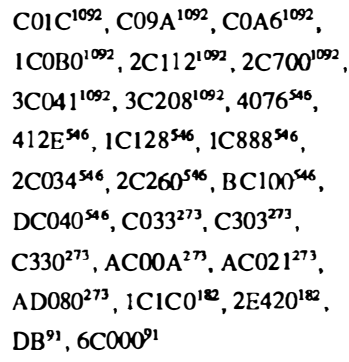 \\
\hline $\begin{array}{c}A(17,6,5)=68 \\
{[P S L(2,16)]}\end{array}$ & 4080 & $\begin{array}{l}(1,6,13,5,4,2,15,10,14,12,3,9,7,11,8) \\
(1,16)(2,3)(4,5)(6,7)(8,9)(10,11)(12,13)(14,15) \\
(2,3)(4,9)(5,7)(6,8)(10,14)(11,13)(12,15)(16,17)\end{array}$ & C.E $E^{68}$ \\
\hline $\begin{array}{l}A(17,6,8) \geq 184 \\
{[Q(16)]}\end{array}$ & 16 & $\begin{array}{l}(1,2, \ldots, 8)(9,10, \ldots, 16) \\
(1,9,5,13)(2,16,6,12)(3,15,7,11)(4,14,8,10)\end{array}$ & $\begin{array}{l}195 \mathrm{~B}^{16}, 1 \mathrm{C} 37^{16}, 2 \mathrm{DOF} \mathrm{F}^{16} \\
5267^{16}, 6857^{16}, 8 \mathrm{~A} 4 \mathrm{~F}^{16} \\
\mathrm{C} 41 \mathrm{~F}^{16}, 10337^{16}, 1492 \mathrm{~B}^{16} \\
18547^{16}, 1 \mathrm{~A} 02 \mathrm{~F}^{16}, 6633^{4} \\
\mathrm{~F} 1^{2}, 5555^{2}\end{array}$ \\
\hline $\begin{array}{l}A(18,6,8) \geq 248 \ddagger \\
{\left[2^{4} .2\right]}\end{array}$ & 32 & $\begin{array}{l}(1,2)(3,4)(5,6) \ldots(15,16), \\
(1,3)(2,4)(5,7)(6,8)(9,11)(10,12)(13,15)(14,16), \\
(1,5)(2,6)(3,7)(4,8)(9,13)(10,14)(11,15)(12,16), \\
(1,9)(2,10)(3,11)(4,12)(5,13)(6,14)(7,15)(8,16), \\
(3,4)(7,8)(9,13)(10,14)(11,16)(12,15)(17,18)\end{array}$ & $\begin{array}{l}11593^{32}, 11 \mathrm{ACC}^{32}, 31472^{32} \\
147 \mathrm{D}^{16}, 172 \mathrm{~B}^{16}, 17 \mathrm{D}^{16} \\
1 \mathrm{D} 47^{16}, 1 \mathrm{E} 1 \mathrm{E}^{16}, 555 \mathrm{~A}^{16} \\
3111 \mathrm{E}^{16}, 31247^{16}, 11 \mathrm{EE}^{8} \\
12 \mathrm{~B}^{8}, 333 \mathrm{C}^{8}\end{array}$ \\
\hline$A(18,6,9) \geq 304$ & 384 & $\begin{array}{l}(2,3)(6,7)(9,13)(10,15)(11,14)(12,16) \\
(2,11,12)(3,16,14)(4,6,7)(5,13,9)(8,10,15) \\
(2,5,3,8)(6,7)(9,16,15,11)(10,12,13,14)(17,18) \\
(1,2)(3,4) \ldots(17,18)\end{array}$ & $\begin{array}{l}103 \mathrm{~F} 3^{\%}, 11 \mathrm{~B} 1 \mathrm{~B}^{\%} \\
10 \mathrm{~F} 69^{48}, 1 \mathrm{FCA}^{32} \\
307 \mathrm{AC}^{32}\end{array}$ \\
\hline $\begin{array}{l}A(19,6.9) \geq 504 \\
{\left[2^{4} \cdot S_{3}\right]}\end{array}$ & 96 & $\begin{array}{l}\text { the generators for } A(18,6,8) \geq 248 \text { together with } \\
(2,3,4)(6,7,8)(10,11,12)(14,15,16)(17,18,19)\end{array}$ & $\begin{array}{l}3162 \mathrm{E}^{96}, 103 \mathrm{CF}^{48}, 11 \mathrm{B2} 7^{48} \\
11 \mathrm{E} 4 \mathrm{~B}^{48}, 13369^{48}, 13535^{48} \\
135 C \mathrm{CA}^{48}, 70356^{48}, 10 \mathrm{~F} 55^{24} \\
111 \mathrm{BB}^{24}, 114 \mathrm{D} 7^{24}\end{array}$ \\
\hline
\end{tabular}

optimizers that work on the principle of "cut a hole in the code, then refill it." The additional step of "pushing" codcwords in order to artificially "expand" the holc can further increase the power of the heuristic.

The codes labeled " $y$," " $y a$," or " $y d$ " in Table I were obtained by one or more of the previous methods. Those with fewer than 1500 words are listed in full in Table
XVI. Most of these codes are at least 2-optimal. They are described in compressed notation, obtained as follows. The words are first sorted into lexicographic order, yielding a sequence of words $c_{1}, c_{2}, \cdots, c_{M}$ (say). The comprcssed notation for this code is $\left(\alpha_{1}, \alpha_{2}, \cdots, \alpha_{M}\right)$. To find $\alpha_{i}(1 \leq i \leq M)$, let $u_{1}, u_{2}, \cdots$ be the list of all vectors, arranged in lcxicographic order and following $c_{i-1}$ in the 
TABLE XV (Continued)

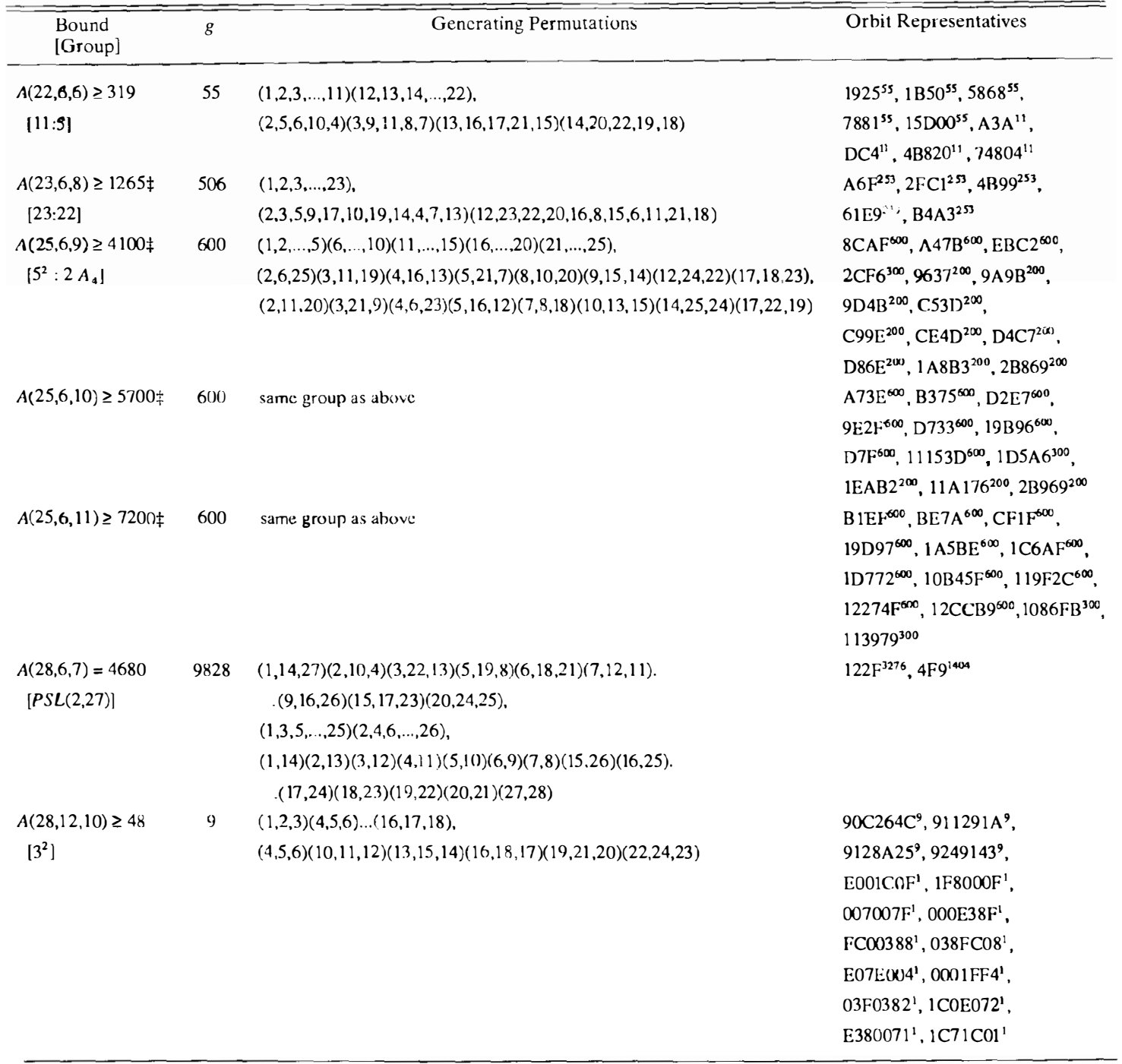

lexicographic order, that have distance $\geq d$ from the subcode $\left\{c_{1}, \cdots, c_{i-1}\right\}$. If $c_{i}=u_{r}$ we set $\alpha_{i}=r-1$. Informally, given $c_{1}, \cdots, c_{i-1}$, we must skip $\alpha_{i}$ lexicographic words to get $c_{i}$. Koschnick [111], who independently discovered this compressed notation, refers to $\left(\alpha_{1}, \cdots, \alpha_{M}\right)$ as a skip-vector for the code.

For examplc the skip-vector for a lexicographic codc itself is simply $0,0, \cdots, 0$ ( $M$ times), which we abbreviate to $0^{M}$.

The algorithm for decompressing this notation is cqually simple. To recover a code $\left\{c_{1}, \cdots, c_{M}\right\}$, showing that $\Lambda(n, d, w) \geq M$, first form the sequence $\alpha_{1}, \cdots, \alpha_{M}$ by cxpanding each symbol $a^{k}$ to $a, a, \cdots, a$ ( $k$ times). Then $c_{l}(1 \leq i \leq M)$ is the $\left(\alpha_{i}+1\right)$ st vector $c$ in lexicographic order starting at $c_{i-1}$ such that $w t(c)=w$ and the distance from $c$ to $\left\{c_{1}, \cdots, c_{i-1}\right\}$ is at least $d$.

In a sense the codes in Table XVI are our failures. At least one of the authors (NJAS) believes that every value of $A(n, d, w)$ in the range of our tables should be attained by a code with some mathematical structure. Experience has shown that sooner or later most random codes in this range are superseded. We hope this will happen to the codes in Table XVI.

\section{ERrata in Earlier Works}

In [13], Table II-D, $A(16,10,7)=A(16,10,9)=4$ (not 3). In Table III-A, $T(1,2,7,16,10)=8$ (not $\leq 6)$, $T(1,3,7,16,10)=12$ (not $\leq 9), T(1,4,7,16,10)=16$ (not $\leq 12), T(1,5,7,16,10)=16$ (not $\leq 15)$. In Table III-D, $T(2,4,7,16,10) \geq 19$ (not $\leq 18)$. We do not at present know how these errors affect the upper bounds in [13] (nor papers such as [180] that make use of these bounds). Until further checks are madc, all the upper bounds in [13] for codes with $d=10$ obtained by linear programming should be regardcd with suspicion. We have also been 


\begin{tabular}{|c|c|c|c|c|c|}
\hline 01111 & 00000 & 00000 & 00000 & 00000 & 001 \\
\hline 00000 & 11111 & 00000 & 00000 & 00000 & 000 \\
\hline 00000 & 00000 & 11011 & 00000 & 00000 & 100 \\
\hline 00000 & 00000 & 00000 & 11111 & 00000 & 000 \\
\hline 00000 & 00000 & 00000 & 00000 & 10111 & 010 \\
\hline 10000 & 00000 & 10000 & 10000 & 10000 & 001 \\
\hline 01000 & 01000 & 01000 & 01000 & 01000 & 000 \\
\hline 00100 & 00100 & 00100 & 00100 & 00100 & 000 \\
\hline 00010 & 00010 & 00000 & 00010 & 00010 & 100 \\
\hline 00001 & 00001 & 00001 & 00001 & 00001 & 000 \\
\hline 10000 & 01000 & 00100 & 00010 & 00001 & 000 \\
\hline 01000 & 00100 & 00010 & 00001 & 10000 & 000 \\
\hline 00100 & 00010 & 00001 & 10000 & 01000 & 000 \\
\hline 00010 & 00001 & 10000 & 01000 & 00100 & 000 \\
\hline 00001 & 10000 & 01000 & 00100 & 00010 & 000 \\
\hline 10000 & 00100 & 00001 & 01000 & 00010 & 000 \\
\hline 01000 & 00010 & 10000 & 00100 & 00001 & 000 \\
\hline 00100 & 00001 & 01000 & 00010 & 10000 & 000 \\
\hline 00010 & 10000 & 00100 & 00001 & 01000 & 000 \\
\hline 00001 & 01000 & 00010 & 10000 & 00100 & 000 \\
\hline 10000 & 00010 & 01000 & 00001 & 00100 & 000 \\
\hline 01000 & 00001 & 00100 & 10000 & 00010 & 000 \\
\hline 00100 & 10000 & 00010 & 01000 & 00001 & 000 \\
\hline 00010 & 01000 & 00001 & 00100 & 10000 & 000 \\
\hline 00001 & 00000 & 10000 & 00010 & 01000 & 010 \\
\hline 10000 & 00001 & 00010 & 00100 & 01000 & 000 \\
\hline 00000 & 10000 & 00001 & 00010 & 00100 & 001 \\
\hline 00100 & 01000 & 10000 & 00001 & 00010 & 000 \\
\hline 00010 & 00100 & 01000 & 10000 & 00000 & 010 \\
\hline 00001 & 00000 & 00100 & 01000 & 10000 & 100 \\
\hline 11000 & 10000 & 00000 & 00000 & 00000 & 110 \\
\hline 00000 & 00100 & 00000 & 00000 & 01001 & 101 \\
\hline 00000 & 00010 & 00110 & 00000 & 00000 & 011 \\
\hline
\end{tabular}

unable to recover the "miscellaneous construction" of [13] that produced $A(18,6,6) \geq 144$, and in the present paper havc rcplaced it by $A(18,6,6) \geq 132$. In [13], p. 89 , col. 2, the words "equivalent to determining $D\left(t, k, v^{\prime}\right)$, where" are illegible in some copies. In (5), linc 5, change 197 to 297.

In [44. (5)], the range is $a^{\prime}<a, b^{\prime}<b$. On page 348 the last line of Table XIII should read $\Lambda(24,10,12) \geq 80$.

In [45], p. 141, caption to Fig. 5.1, the length of code is $11($ not 10$)$

In [50], p. 12, line $21,\left|\Gamma_{12}\right|=112952\left(\right.$ not ||$\left._{10} \mid\right)$.

In [72], p. $40, T(1,6,6,15,10)=8$ (not 7). On p. 40, column 1, linc 3 , change 554 to 553. In Table IV, $A(16,10,7)=A(16,10,9)=4($ not 3$)$.

In [147], $A_{9}^{2}=56$ (not 99).

In [150], Table $2, A(19,4,5) \geq 644$ (not 664).

\section{ACKNowledgment}

We thank several correspondents who kindly sent us codes they had discovered. Since some codes were found independently by several people, and others have been transformed or "beautified" (and so do not appcar in the final table in their original form) it is appropriate to rccord these constructions here. To keep this list to manageable size we mention only codes that are as good as the current record, were unpublished at the time of writing, and wcre found since the publication of [13].

\begin{tabular}{|l|l|l|l|l|}
\hline 1000 & 1000 & 1100 & 0101 & 1001 \\
1000 & 0100 & 0011 & 1010 & 1001 \\
1000 & 0010 & 0011 & 0101 & 0110 \\
1000 & 0001 & 1100 & 1010 & 0110 \\
\hline 0100 & 0110 & 1000 & 1100 & 0101 \\
0100 & 0110 & 0100 & 0011 & 1010 \\
0100 & 1001 & 0010 & 0011 & 0101 \\
0100 & 1001 & 0001 & 1100 & 1010 \\
\hline 0010 & 1010 & 0110 & 1000 & 1100 \\
0010 & 0101 & 0110 & 0100 & 0011 \\
0010 & 1010 & 1001 & 0010 & 0011 \\
0010 & 0101 & 1001 & 0001 & 1100 \\
\hline 0001 & 0011 & 1010 & 0110 & 1000 \\
0001 & 1100 & 0101 & 0110 & 0100 \\
0001 & 1100 & 1010 & 1001 & 0010 \\
0001 & 0011 & 0101 & 1001 & 0001 \\
\hline 1111 & 1111 & 0000 & 0000 & 0000 \\
\hline Fig. 2. $A(20,10,8)=17$.
\end{tabular}

T. Etzion and C. L. M. van Pul [68] showed $A(17,4,6)$ $\geq 854$,

H. Hämäläinen [80] showed $\iota^{(18,6,9) \geq 304, A(19,6,9)}$ $\geq 504, A(23,10,9) \geq 45, A(25,10,8) \geq 48, A(26,14,11)=$ $10, A(27,14,11)=13, A(28,12,8) \geq 19, A(28,12,10) \geq 48$.

I. Honkala [91] showed $A(22,12,9)=8, A(25,12,8)=$ 10. I. Honkala [93] showed $A(26,14,9)=6, A(26,14,10)$ $=8, A(28,14,9)=7$.

M. K. Kaikkonen [105] showed $A(22,9) \geq 68, A(25,8,9)$ $\geq 829, \quad A(21,10,9) \geq 27, \quad A(23,10,8) \geq 33, \quad A(24,10,9) \geq$ $56, \quad A(25,10,6) \geq 10, \quad A(25,10,8) \geq 48, \quad A(26,10,6) \geq 13$, $A(27,10,6) \geq 14, \quad A(25,12,8) \geq 10, \quad A(28,12,14) \geq 106$, $A(26,14,10)=8, \quad A(26,14,11)=10, \quad A(27,14,11)=13$, $A(28,14,9)=7, \quad 1(28,14,11) \geq 21$,

C. L. M. van Pul [149] showed $A(17,4,5) \geq 424$, $A(17,4,6) \geq 854, \quad A(19,6,7) \geq 338, \quad A(19,6,8) \geq 408$, $A(21,6,7) \geq 570, \quad A(17,8,8)=34, \quad A(18,8,6)=21$, $A(22,10,7) \geq 16, A(22,10,8) \geq 24$, C. L. M. van Pul [150] showcd $A(17,6,7) \geq 166, A(18,6,7) \geq 243$, and

S. Rankinen [151] showed $A(20,6,8) \geq 588$.

After this paper was submitted wc rcceived a preprint by K.-U. Koschnick [111], which independently showed $A(12,4,5) \geq 80, A(18,8,6)=21, A(22,10,7) \geq 16$, and improved one of our entries by showing $A(23,10,7) \geq 20$. A code with the lattcr parameters and equivalent to Koschnick's is given in Table XVI.

Other codes were found by Chen, Jin, and Fan [34], Cheswick [35], Chung and Kumar [37], Darwish and Bose [49], Dueck and Scheuer [63], Lin [123], Zaptcioglu [184] and Zinoviev and Litsyn [188]. We thank all of these correspondents.

David Johnson kindly allowed us to use his simulated annealing graph coloring programs [98]. We also thank John Conway for many helpful discussions. Iiro Honkala, Heikki Hämäläinen and Markku Kaikkonen for informing us of a large number of codes that they had found, and Tuvi Etzion and Kevin Phelps for several helpful comments. Tuvi Etzion also helpcd us find some of the partitions in Tablc VI (cf. [65]). Aaron Grosky and Ralph Knag provided valuable assistance in running our programs at Bell Labs.

The automorphism groups of certain codes in this paper wcrc computed using B. D. McKay's graph-automor- 
TABLE XVI

Codes with no Kvolvn Structure, Describfin by Skip-Vectors

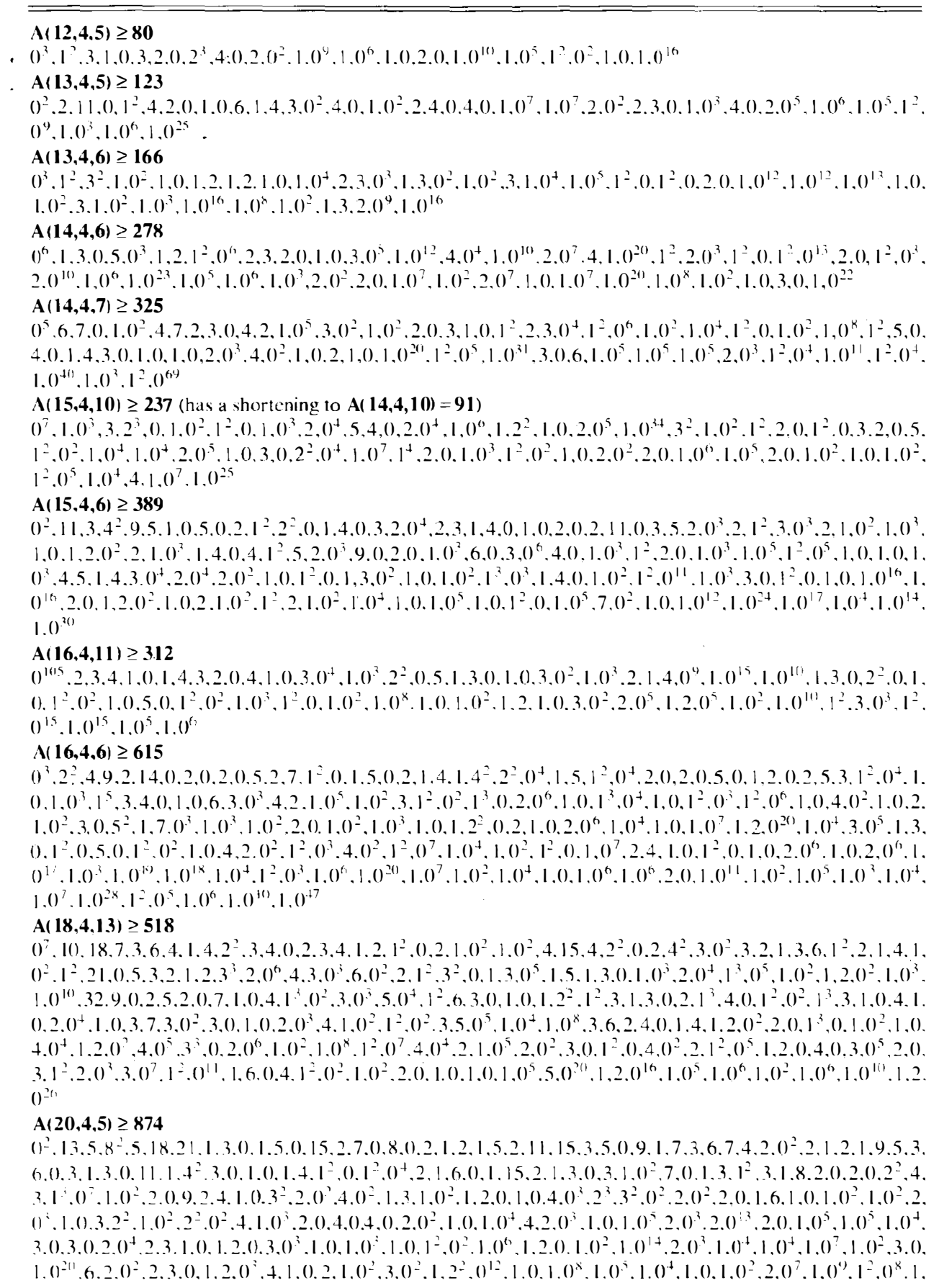


TABLE XVI (Continued)

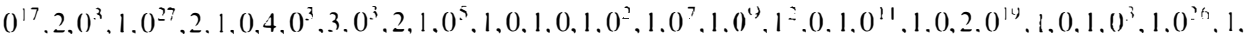

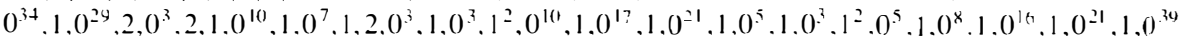

\section{$\mathbf{A}(\mathbf{1 8 , 6 , 8 )} \geq \mathbf{2 6 0}$}

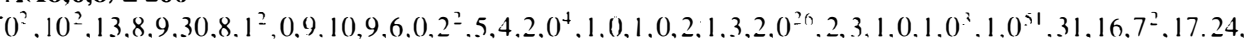

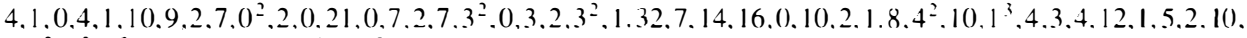
$0.3^{2}, 1^{2},\left(0^{2} \cdot 7 \cdot 2 \cdot 0.1 \cdot 0^{4} \cdot 1 \cdot 0^{9} \cdot 1.0^{59}\right.$

$A(21,6,8) \geq 774$

$139,694,44,3,214.933,307,286,778,117,622.712 .220,438,242,328,471.22 .4 .55,10.0 .62,23,2.1 .2 .10 .0$.

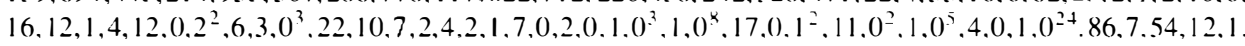

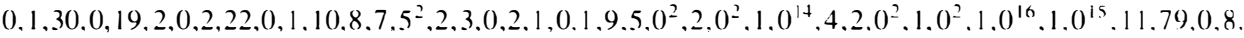

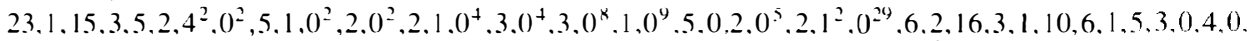

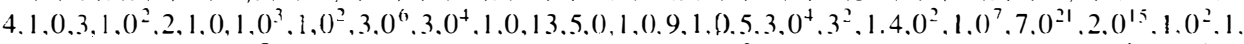

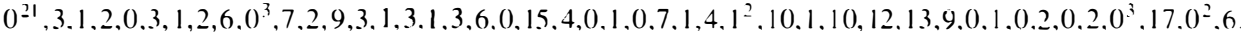

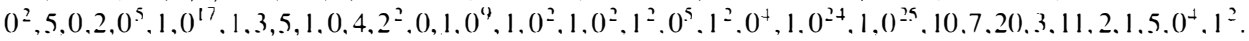

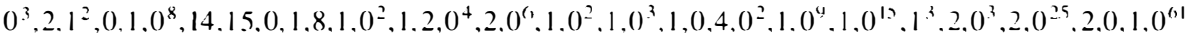

\section{$\mathbf{A}(21,6,9) \geq 1184$}

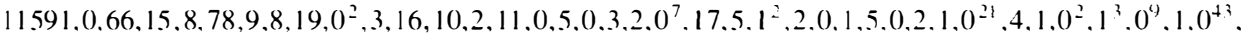
$46,1,49,1,29,8,28,2,0^{3}, 1,6,0,1,6,0^{6}, 1,0^{4}, 1,4,0^{2}, 1,0^{4}, 1,0^{27}, 1,0^{56}, 1.4 .0,1,2,0^{2}, 1,0,2,0^{2}, 1,0,3,14,0,11$, $4,18,0,2^{2}, 1.0^{111}, 16,1^{2}, 0^{3}, 6,0,1,2,0,3,0,1,0^{2}, 5,6,12,4.6,10.2,0^{2}, 8.2,0,1,4.3,0^{2}, 1,0.4 .0 .2,0,1^{2}, 0.19,0^{5}$. $1.2 .0^{5}, 1,0^{106} \cdot 61,0,7,0.1^{2}, 0.2^{2}, 0^{4}, 1^{2}, 2,0^{3}, 3,1,0,1,0^{2}, 1.0^{3}, 1^{2}, 0,1,0,1,0,1,0^{4}, 1.2 .0^{7}, 1.0^{3}, 2^{2}, 0^{3}, 2^{2}, 1$.

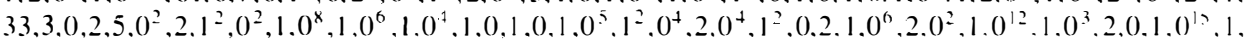

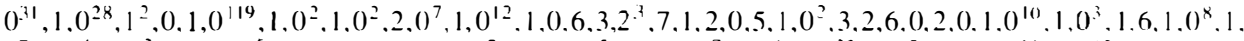
$0^{7}, 1,0^{4}, 3,0^{2}, 1,0,1,0^{5}, 1,0,1,0,2,6,3,0,1^{2}, 0,1,2,0^{5}, 2,4,1,0^{7}, 2,0^{6}, 1,0^{3.3}, 1,0^{5}, 1,0,1,00^{11}, 1,00^{13}, 2.0 .3,0.1$ $0^{3}, 1,0^{37}, 1,0^{50}$

\section{$A(21,6,10) \geq 1454$}

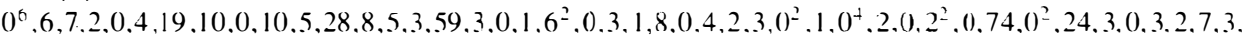

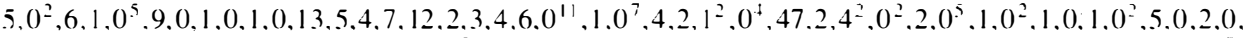

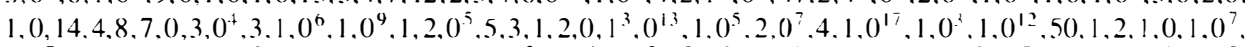

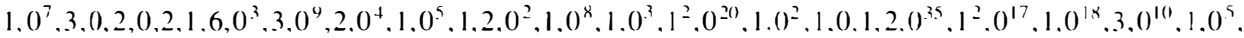

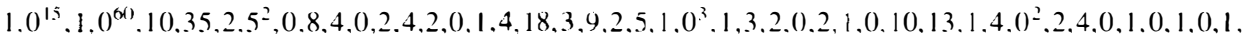

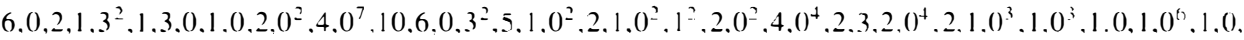

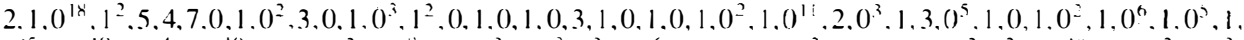

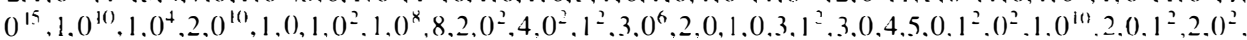
$3,1,0,1^{2}, 2,0^{1,3}, 1^{2}, 0^{2}, 1,2,0^{7}, 1,0,1,0^{5}, 1^{2}, 0,1,0,1,2,1,0,2^{2}, 00^{15}, 1,0^{2}, 1,2,0^{45}, 1^{2}, 00^{14}, 1,3,0,1,0,2^{2}, 00^{33}, 1$. $0^{55} \cdot 2,1,0^{14} \cdot 1,0^{49} \cdot 4 \cdot 0,1,0,1,0^{24} \cdot 1 \cdot 0^{29} \cdot 1^{2} \cdot 00^{39} \cdot 1,0^{42} \cdot 1.0^{53} \cdot 1.0^{1.37}$

$\mathbf{A}(22,6,8) \geq 1139$

$139.694 .443,214,933,307,286.778 .117 .622 .712 .220 .438 .242,328.471,22,4.55,10.0 .62 .23,2.1 .2 .10 .0$.

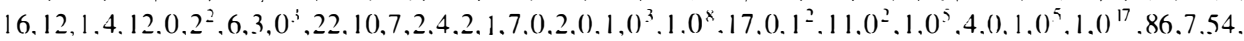

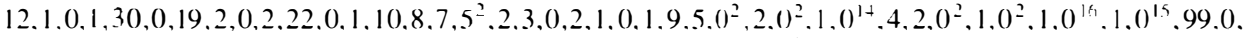

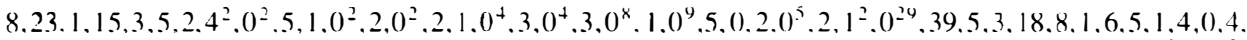

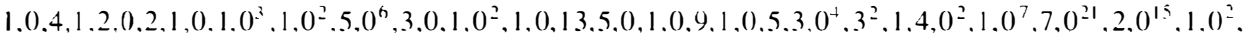

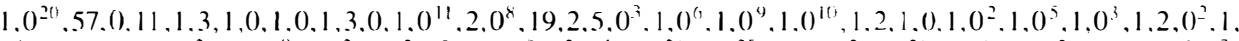

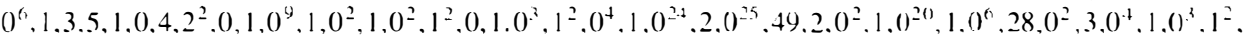

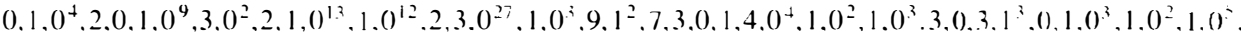

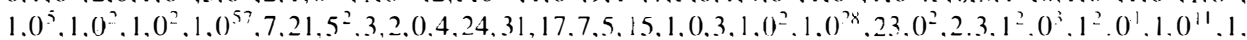
$0)^{2} \cdot 1,0^{2} \cdot 1,0^{31} \cdot 2,\left(0^{26} \cdot 3,0^{4}, 4 \cdot 0^{3 \times} \cdot 1,0^{55}\right.$

\section{$\mathrm{A}(\mathbf{2 3 , 6 , 8}) \geq 1436$}

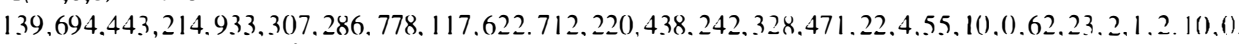

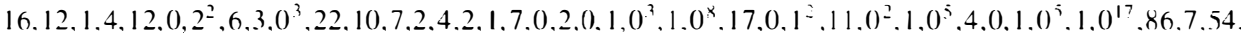

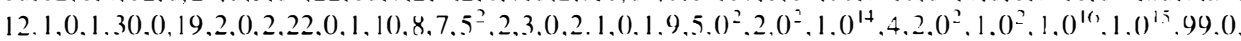

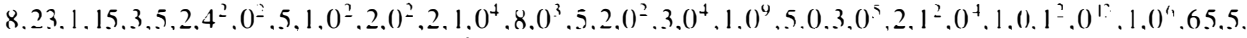

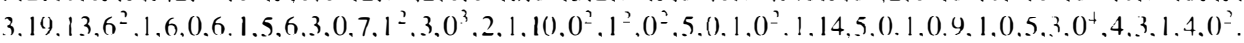

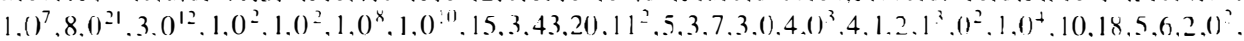

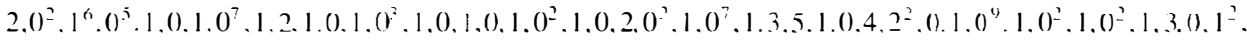


TABI E XVI (Continued)

$0^{2}, 1^{2}, 0^{4}, 1,0^{24}, 2,0^{25}, 52,2,1,0,1,0^{2}, 2,0,1,0^{12}, 3,1,0^{2}, 1,35,0^{2}, 3,0^{4}, 1,3,0,2,1,2,0,1,0,1,2,1,0^{5}, 1^{2}, 0^{2}, 3,2$, $0,3,1,2^{2}, 0^{4}, 1,0^{2}, 1^{2}, 0^{2}, 1,0,1,0,1,3,0^{2}, 1,0,1,2,1,0,1,0^{6}, 2,0^{4}, 6,11,4,0^{2}, 6,0^{3}, 1,0^{2}, 1,0^{2}, 3,0^{2}, 2,0^{4}, 1,0,1$, $0^{3}, 1,3,0,3,0,3,1^{3}, 0,1,0^{3}, 1,0^{2}, 1,0^{5}, 1,0^{5}, 2,0^{2}, 1,0^{2}, 1,0^{5}, 1,0^{16}, 2,0,1,0^{2}, 2,0^{15}, 1,0^{9}, 2,4,2,11,0^{2}, 3,2,17$. $1^{2}, 6,0^{2}, 7,1,0^{3}, 14,4,2,6,7,2,7,20,0,5,1,0,1,11,2,5,0,1^{2}, 0^{3}, 1,0^{12}, 1,0^{4}, 31,0^{3}, 1^{2}, 2,0^{3}, 1,0^{2}, 2,0^{2}, 1,0^{3}, 3^{2}$. $0^{15}, 2,0^{4}, 1,0^{2}, 1,0^{2}, 1,0^{3}, 2,0^{2}, 1,0^{2}, 1,0,2,0^{2}, 2,1,2,0^{2}, 2,1,0,2^{2}, 0,6,0^{4}, 1,0,1,0^{6}, 1,0,1,0^{4}, 2,1,0^{5}, 1,0^{2}, 1$. $0^{12}, 1,0^{8}, 1,0^{7}, 1,0^{12}, 2,1,0^{22}, 1,0,1^{2}, 0^{20}, 1,0^{2}, 1,0,1,2,1,5,1,0^{2}, 7,2,0,1,0^{2}, 1,2,0^{2}, 1^{2}, 0,3,0^{4}, 1,0,1,0^{2}, 2$, $0^{5}, 1^{2}, 0^{2}, 2^{2}, 1,0,1,0,1,0^{3}, 1,0^{2}, 2,0^{2}, 1,0^{4}, 1,0^{3}, 1,2,1,2,3,0,2,4,3,2,4,0,2,0,3,2,1^{3}, 0,1,3,1,0^{2}, 1,0,2,0^{3}, 1$, $0,2,0,1,2,0,3,0^{3}, 1,0,4,0^{2}, 1,0^{7}, 1^{2}, 2,1^{4}, 0^{7}, 1,3,0^{4}, 3,1,0^{2}, 1,0^{2}, 1,2,0^{3}, 1,3,2,0,4,0,1,0^{2}, 1^{2}, 2,0^{5}, 3,0^{3}, 1,0$, $1,0,1,0^{3}, 1,0^{4}, 1^{2}, 0^{4}, 1,0^{2}, 7,2,0^{3}, 1^{4}, 0^{5}, 1,0^{2}, 1,0^{10}, 1,3,2,0^{10}, 2,0^{21}, 2,0^{4}, 1,0^{3}, 5,0,1,0,2,0^{4}, 1^{2}, 0^{4}, 1^{2}, 0^{2}$. $1^{2}, 2,0^{21}, 1,0^{14}, 1,0^{5}, 1,0^{32}$

$A(28,6,5) \geq 272$

$0^{2}, 148,162,22,123,80,17,71,291,299,220,5,37,183,19,28,123,9,90,55,9,260,121,70,8,101,1,11,46,48$, $75,2,4,31,21,6,24,2,14,0^{2}, 48,7,58,4,1,41,35,1,17,2,12,0,9,19,7,1,2,9,66,35,3^{2}, 5,9,0^{2}, 7,2,8,3,2,15,6$, $0,3,1,15,3,13,0,3,1,0,4,0,9,2,0,1,0^{3}, 15,16,3,11,0,15,7,0,18,0,7,2^{2}, 4,1,0,1,0^{3}, 1,0^{3}, 11^{2}, 5,2,4,11,2^{2}, 3$, $1,0,3,5,2,0,2,0^{2}, 1,0^{3}, 1,0,1,0^{6}, 7,0,5,0,5,3,10,12,4,1,0,3,0,4,0,2,0,1^{2}, 0,1,0^{4}, 1,0^{2}, 1^{2}, 0^{4}, 1,4,0,1^{3}, 7,2$, $0,3,1,0^{5}, 1,0^{2}, 1,0^{4}, 1,0^{2}, 1,0^{6}, 1,0^{4}, 3,0^{3}, 1,2,1,0^{2}, 1,0,2,1,0^{12}, 3,0^{24}$

\section{$\mathrm{A}(25,8,10) \geq 1248$}

$196702,124,0^{2}, 22,0,5,3,0,8,0^{5}, 6,0^{2}, 3,0^{5}, 18,7,0^{2}, 2,0,1,0^{2}, 2,0^{5}, 2,0^{8}, 6,0^{23}, 12,0^{87}, 850,0^{23}, 10,0^{23}, 4$. $0^{23}, 8,0^{87}, 478,0^{2.3}, 4,0^{23}, 2,0^{23}, 4,0^{87}, 444,0^{15}, 8,0^{15}, 4,0^{47}, 192,0^{159}, 240,0^{79}, 120,0^{159}, 1138,0^{15}, 162,0^{135}$. $17,15,7^{2}, 0,2,3,0^{9}, 12,0^{39}, 16,6,0,14,0^{3}, 1,0^{7}, 14,0^{24}, 23,1,4,5,0^{12}, 12,0^{23}$

$\mathbf{A}(\mathbf{2 6}, \mathbf{8}, 19) \geq \mathbf{2 5 7}$

$30027,1557,7514,6608,3164,1865,3262,695,400,12,243,460,241,614,94,0,391,195,111,212,252,1065$, $316,234,17,32,140,1833,132,8,0,483,34,5,0,3,9,4,0^{2}, 10,0^{2}, 13,188,0,7,8,21,1,0,3,0,1,2^{2}, 0^{3}, 1,4,0,1,4$, $1,2,1,0,1,0,1,0^{3}, 1,0,2,0^{5}, 1,0,11,0,1^{2}, 0^{5}, 1,0^{10}, 1,0,1,0^{5}, 3,0^{17}, 1,0^{18}, 1,0^{23}, 1,0^{47}, 1,0^{3}, 1,0^{10}, 1,0,2,0^{x}$, $1^{2}, 0^{8}$

\section{$\mathrm{A}(\mathbf{2 6 , 8 , 9 )} \geq \mathbf{8 8 3}$}

$0^{4}, 1,9,2^{2}, 0,6,2,1,7,0,113,65,138,32,409,5,0,53,0,5,11,0,3,9,51,43,0,1,33,0,25,4,0^{2}, 39,0,4,6,0^{9}, 5,52$. $30,0^{2}, 5,0^{2}, 1,0^{6}, 2,4,2,4,0^{2}, 6,0^{15}, 30,3,0^{5}, 1,0^{3}, 2,0^{7}, 1^{2}, 0^{7}, 1,0^{13}, 1,0^{18}, 21,14,0^{3}, 1,0,1,0^{3}, 1,0,1,0^{17}, 1$, $0^{7}, 1,0^{8}, 1,0^{3}, 1,0^{16}, 1,0^{24}, 1,0^{5}, 1,0^{5}, 1,0^{4}, 2,0^{6}, 1,0^{6}, 1,0^{8}, 1,0^{8}, 1,0,2,0^{16}, 1,0^{7}, 1^{2}, 0^{2}, 1,0^{5}, 1,0^{12}, 1,0^{24}, 1$, $0^{5}, 1,0^{16}, 1^{2}, 4,0^{2}, 1,0^{2}, 1,0^{3}, 1,0^{3}, 1,0,2,0^{4}, 1,0,1,0^{32}, 1,0^{5}, 1,0^{4}, 1,0^{6}, 1,0^{7}, 1,0^{11}, 1,0^{3}, 1^{2}, 0^{11}, 2,0^{5}, 1,0^{20}, 1$, $0^{17}, 1,0^{11}, 1^{2}, 0^{12}, 1,0^{2}, 1,0^{11)}, 1,0^{15}, 5,10,0^{2}, 1^{2}, 0^{2}, 4,2,1,0,1,5,0^{2}, 2,1,0,1,2,4,1,0,2,1^{2}, 3,1,4,0,2,0^{2}, 1,5$, $1^{3}, 0,1^{2}, 0,3,1,0^{3}, 1,2,0^{2}, 2,0^{2}, 1,2^{2}, 3,0,1,0,1,0,1,0,3,1,0,2^{2}, 0,2,0^{3}, 1,3,4,1,0^{8}, 2,0,1,0,1,0,1,0^{2}, 4,3,0$, $1^{3}, 0^{2}, 1,0,1,2^{2}, 1^{4}, 0,1^{2}, 0^{3}, 1^{2}, 6,1,0^{2}, 1,0^{4}, 1,0^{3}, 2,1,0^{2}, 1,0,1^{2}, 0,3,0,2,1,0,1,3,2,0,1,3,0,1^{2}, 0,2^{2}, 0^{3}, 3$, $0^{2}, 3,0,2,0,3,0^{3}, 3,0^{3}, 2,0^{6}, 3,0^{2}, 1,3,0^{4}, 3^{2}, 0^{4}, 1,0^{6}, 1,0,1,8,1,6,0,6,0,1,0^{2}, 1,0,1,0^{33}, 1^{2}, 0^{22}$

\section{$\mathrm{A}(27,8,7) \geq 278$}

$2273,164,5,224,161,154,40,2,44,135,0^{2}, 45,4,9,15,2^{2}, 8,4,7,12.100,13,0^{2}, 8,0^{2}, 2,4,3,2,0,4,0,9,0^{3}, 2,0^{3}$, $1,3,2,0^{8}, 9,22,0,4,0^{4}, 1,0,1,0,1^{2}, 0,1,0^{7}, 1,0^{3}, 1,0^{3}, 12,0^{10}, 1,0^{11}, 1,0,2,0^{8}, 1^{2}, 0^{4}, 1^{2}, 0^{5}, 11,5,0,1^{3}, 0^{3}, 1^{4}, 0$, $1,0^{3}, 1,0^{4}, 1,0^{4}, 1,0,1,0^{3}, 1^{2},()^{2}, 2,1.0^{3}, 1,0^{3}, 1,0^{2}, 1,0^{2}, 1,0,15,48,13,4,6,8,12,17,26.1,53,43,16,8,14,13$, $0,4,0,6,7^{2}, 4,5,0,1,0,15,3,8,25,6^{3}, 0^{2}, 12,8,1,3,0^{5}, 2,1,0^{6}, 4,0^{3}, 4,0^{3}, 1,0,2,0^{7} \cdot 1,0^{18}$

$\mathrm{A}(\mathbf{2 7}, \mathbf{8}, 19) \geq \mathbf{7 6 6}$

$1,0,1,0^{7}, 1^{2}, 0^{59} \cdot 1,0^{12} \cdot 1,0^{681}$

$\mathrm{A}(\mathbf{2 7}, \mathbf{8 , 9 )} \geq \mathbf{9 7 0}$

$0^{4}, 1,9,2^{2}, 0,6,2,1,7,0,3,0,1.9,1^{2}, 0,3,1,0^{2}, 2,0,49,17,84,12,44,64,3,50,163,1,0,43,0.12,8.0,5,0^{3}, 7.2 .0$, $159,6,10,21,0,23,1,7,0^{3}, 1,0^{6}, 30,0,3,0^{3}, 4,0^{10}, 1,8,5,21,30,0^{2}, 1,2,0^{4}, 3,0^{4}, 1,0^{3}, 1,0^{11}, 1.0^{7}, 1,0^{15}, 2,16,1$ $22,0^{2}, 1,0^{3}, 1,0,1,0^{7}, 1,4,0,1,0^{10}, 5,0^{23}, 1,0,1,0^{10}, 1,0^{6}, 1,0^{13}, 11,1^{2}, 4,0^{10}, 1,0,1,2,0^{2}, 1,0^{10}, 2,0^{21}, 1^{2}, 0^{14}$. $1,0^{3}, 1,0^{2}, 1,2,1,0^{8}, 1,0^{3}, 1,0^{8}, 2,0^{5}, 1,0^{5}, 1,0^{13}, 1,0^{16}, 1,0^{2}, 6,0^{16}, 1,0^{8}, 1,0,1,0^{6}, 1,0^{20}, 1,0^{6}, 1,0^{9}, 1,0^{7}, 1$. $0^{16}, 1^{2}, 0^{7}, 1,3,0^{5}, 1,0^{6}, 1,0^{4}, 1,0^{2}, 1^{2}, 0^{5}, 1,0^{2}, 1^{2}, 0^{9}, 1,0^{4}, 2,0^{4}, 1,0,1,0^{8}, 1,0^{4}, 1,0^{4}, 1,0^{2}, 1,0^{6}, 1,0^{8}, 2,0^{12}, 5$, $0^{2}, 1^{4}, 0,1,0,2,0^{3}, 1.0^{2}, 3,0,1,0,1,5,0^{3}, 1,0^{4}, 1^{3}, 2,0,2,0,3,15,0,1^{6}, 0^{4}, 2,1,2,0,1,0^{2}, 4,0^{6}, 2^{2}, 3,1,0^{2}, 3,0,1^{3}$, $5.0^{3}, 2,0^{2}, 1,0,1,0,3,2,0^{5}, 3,0^{2}, 2^{2}, 0,1,0,2,1,2,4,0,1,3,0^{3}, 3,1^{2}, 0,1,0^{2}, 1,3,0,2,1,2,1^{4}, 2,3,2.0^{2}, 1,0^{2}, 1.0^{2}$. $3,1^{2}, 0^{3}, 3,9,0^{2}, 7,1,0^{3}, 1,0,13,8,15,3,0,1^{2}, 11,0,15,3,4,3,2,8,4,8,3,0,1,0^{2}, 1,6,0^{2}, 1,0,1,3,4^{2}, 2,1^{2}, 4,0^{2}$. $3,2,5,0,4,2^{3}, 1^{2}, 0^{4}, 2,0^{3}, 3.0^{4}, 2^{2}, 0,1,0,2,0^{2}, 3,0,1,6,0,2^{2}, 0,1^{2}, 0^{6}, 1,0^{x}, 2,0^{5}, 1,5,1,7,4,1,7,2,0,4,2,0^{3}, 2()$, $17,0,1,3,2,1,0,4,3,0^{5}, 4,0^{3}, 13,0,8,2,1,0^{2}, 12,0^{2}, 3,1^{3}, 2,0^{2}, 2^{2}, 0^{5}, 1,0^{5}, 1,0,1,0,1,0^{6}, 9,1,3,0,1,2,1,5,0^{5}$. $3.0^{4}, 1,0,1,0^{4}, 1,0,1,0^{5}, 1,0^{3}, 1,0,1,0^{3}, 1,0^{13}$

$A(28,8,21) \geq 296$ 
TABLE XVI (continued)

$2616.169 .1019 .39 .293^{2} .484 .166 .831 .33 .369 .134 .377 .17 .34 .69 .1 .215 .2(01.406 .7 .22(0,1.91 .52 .39 .5 .19 .3$. $219.0 .89 .2 .61 .1 .40 .26 .13 .5 .3,10.50 .7 .60 .4 .38 .57 .1 .0 .2 .1 .7 .20 .1 .6 .0 .11 .5 .6^{2} .3 .21 .6 .22 .0 .16 .6 .5 .10 .3$. $16.10 .2^{2} \cdot 6.1 .2 .3 .1 .4,1.50,434,82,4,6.79,17,20,38,29,166,1.7,3,8,6,7.40,24,12,9,27,25,5,12.6,24.6$.

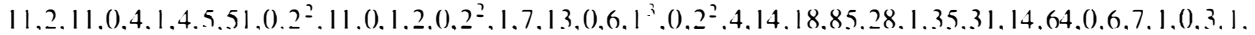

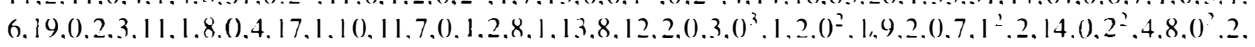

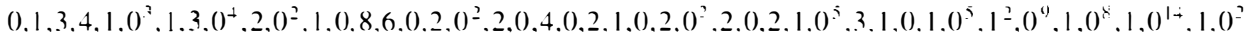
$\mathrm{A}(\mathbf{2 8 , 8 , 2 0}) \geq 833$

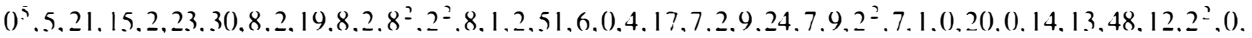
$14.4 .2 .00^{2} .17^{2}, 6,12.24,10.00^{2} .1 .9 .1 .5 .33 .22 .9 .17 .0 .2^{2} .3,2.1 .2 .1 .3 .0 .1 .0 .1 .2 .23 .10 .2 .4 .12 .7 .1 .21 .2 .4 .5$.

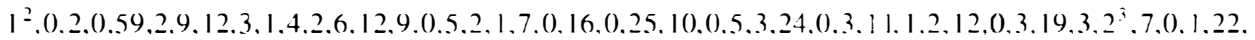

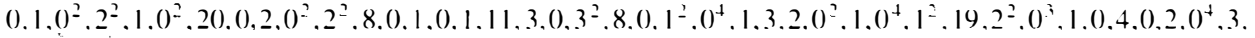

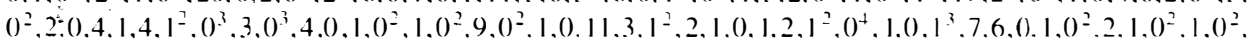

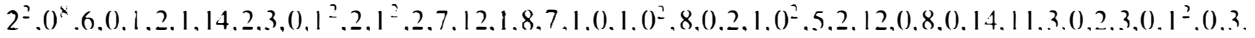

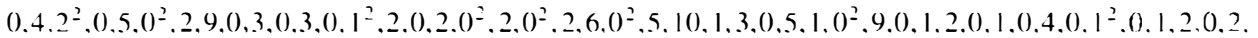

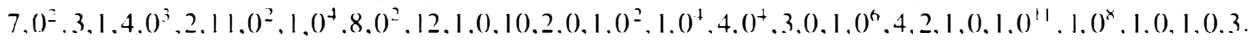

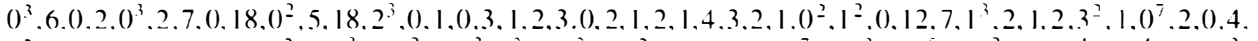

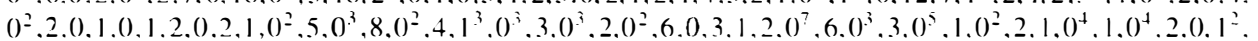

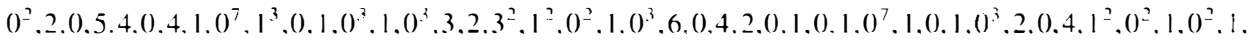

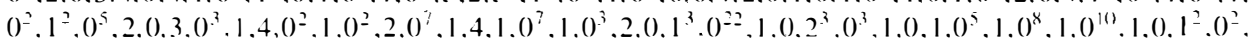
$2.00^{2} \cdot 4.0^{13} \cdot 1.00^{2} \cdot 1 \cdot\left(0^{10} \cdot 1.00^{3} .2^{2} \cdot\left(0^{18}\right.\right.$

\section{$\mathrm{A}(\mathbf{2 8 , 8 , 1 9}) \geq \mathbf{1 1 0 7}$}

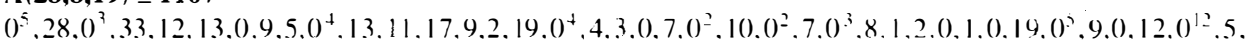

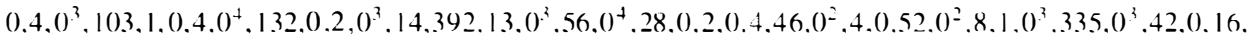

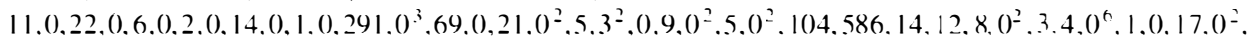
$13 .\left(0^{3} .3 .00^{3} .1 .3 .0 .4 .00^{3} .37 .5 .90 .2 .00^{3} .273 .13 .0 .49 .150 .00^{7} .407 .98 .22 .46 .57 .55 .0^{3}, 82.07 .7 .312 .46 .281 .9\right.$.

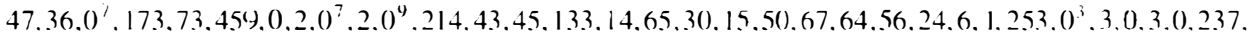
$161.176 .0 .94 .38 .\left(0^{2} .1 .00^{3} .1 .82 .61 .42 .51 .95 .11 .0^{3} .2 .00^{11} .28 .82 .41 .120 .0 .27 .21 .25 .00^{2} .32 .00^{2} .18 .0 .1 .00^{\prime}\right.$.

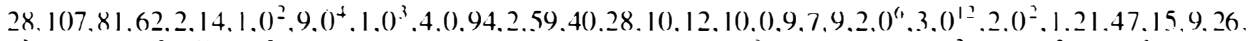

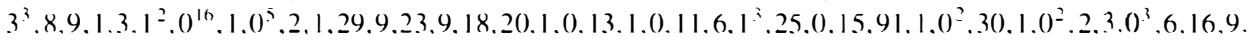

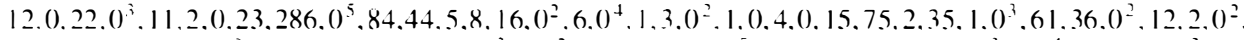

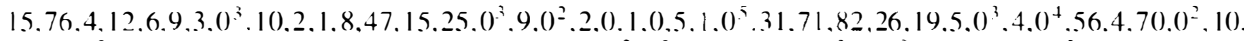

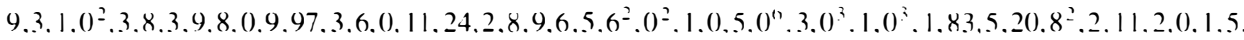

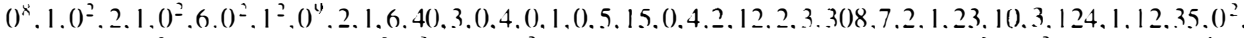

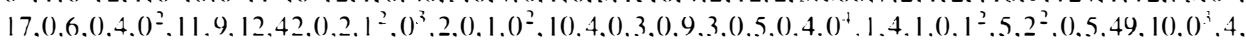

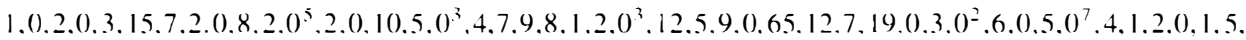

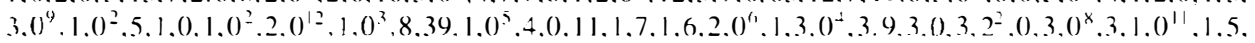

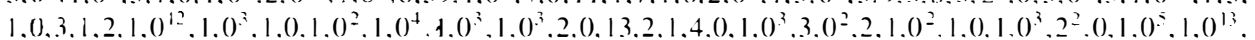

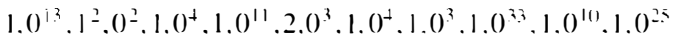

\section{$\mathrm{A}(\mathbf{2 3 , 1 0 , 7 )} \geq 20$}

$310.3\left(0,8098.707 .3722 .291 .84 .959 \cdot 43.9 .2 .39 \cdot\left(0^{3}, 3.00^{5}\right.\right.$

\section{$\mathrm{A}(\mathbf{2 6}, \mathbf{1 0}, \mathbf{1 2}) \geq \mathbf{1 8 5}$}

()$^{4} .9 .804 .16 .34 .31 .0 .653,287.18 .333 .12 .7 .19 .1108 .2 .108 .41 .8 .10 .177 .2 .0 .9 .11 .13 .3 .1 .6 .23 .45 .6 .16 .1$. $0^{2} .21 .0 .18 .4 .25 .0 .5 .21 .1 .0^{2} .1 .3 .1 .2 .1^{2} .00^{2} .1^{2} .0 .52 .18 .3 .6 .7 .0 .12 .4^{2} .2 .0 .1^{2} \cdot 0.7 .00^{2} .2 .0 .2 .00^{1} \cdot 15.9 .2 .1^{2}$ $3.1^{2} .6 .12 .2 .0 .10 .0 .10 .58 .1 .2 .9 .1 .9 .6 .7 .0 .7 .0 .1 .5 .2^{2} .00^{9} \cdot 4.3 .0 .28 .9 .10 .9 .24 .7 .00^{-} .8 .4 .3 .0 .6 .5 .0^{2} .1 .0^{7}$. $54.36 .0 .13 .5 .1 .18 .1^{2} \cdot 2.4 .1 .14 .1 .00^{\circ} .1 .00^{13}$

\section{$A(26,10,13) \geq 191$}

()$^{2} .56 .42(0.4213 .616 .3(042.732 .98 .1(0921.2166 .577 .126(0.0 .299 .606 .249 .4566 .1544 .579 .478 .0 .14 .3 .145$. 1.50.224.361.38.431.267.241,0.34.2033.404.108.1062.0.1172.89.242, 160.371.0.74.22-17.129.101. $105.31 .14 .64 .14 .31 .27 .12 .0 .67 .3 .33 .0)^{2} .11 .3 .94 .119 .63 .15 .1^{2} .6 .40 .75 .69 .30,0.45 .31 .7 .93 .71 .24 .41 .14$. $98.40 .7 .6 .2 .3 .2 .5 .2 .18 .14 .2 .39 .22 .1 .3 .0 .11 .3 .0 .1 .2 .0 .2 .3 .1 .6 .0 .2 .00^{-} .2 .00^{5} .3 .62 .0 .23 .150 .5 .6 .79 .29 .8 .6$.

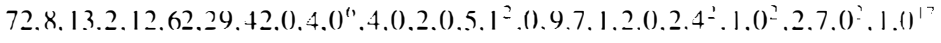

A $(27,10,11) \geq 213$

52263.46674 .39014 .12594 .7191 .49805 .257 .1176 .8505 .2624 .2904 .1024 .411 .5583 .9562 .3356 .214 .1045 $3611.2612 .291 .935 .641 .227 .406 .430 .2926 .3213 .10399 .2(077.44 .486 .200 .22 .156 .171 .481 .18 .3 .51 .412 .27$ 
TABLE XVI (continued)

$93,42,38,91,9,17,3,1410,278,244,96,100,17,33,132,24,10,6,31,106,11,7,4,15,74,11,55,6,3,22,4,8,0,1$. $0,29,0,10,6,11,0,1^{3}, 0^{11}, 190,10,52,15,5,37,3,0,5,2,8,21,1,3,1,0^{2} .15,0^{2} .9,5,0^{4}, 2,0.5,0,1,0^{4}, 1,0^{36}, 47,3$, $1^{3}, 2,5,1,0,1^{2}, 3,2,1,0,1,0^{2}, 2,0^{13}, 1,0^{5}, 1,0^{3}, 1^{2}, 0$

$\mathrm{A}(27,10,12) \geq 257$

$0^{4}, 1212,865,887,143,95.906,134,674,11,56,2,0,44,58,634,6,0,39,157,8,47,14,8^{2}, 23,9^{2}, 50,31,11,0,3^{2}$, $5,17,0^{3}, 5,0,7,0,4,6,0,3,0,5,0^{6} .1,0^{4}, 38,20,9,5,13,3,11,17,2,17,4,0,2,13,4,0^{2}, 3,0^{5}, 1^{2}, 0,2,11,2^{4}, 1,0^{3}, 2$, $0^{2}, 23,42,20,16,10,0,6,3,7,0,2,0^{2}, 13,3,0,1,0,6,0^{6}, 1^{2}, 3,0^{3}, 36,35,4,0,5,0,3^{2}, 4,0^{4}, 7,0,1,0^{6}, 8,11,6,7,1,4$ $3,1^{2} .4,1,0,8,2^{2}, 0,1,0^{2}, 3,0,1,0^{6}, 23,8,2,1,4,2,3,0^{3}, 18,8,12,5,12,1,2,1,4,0,4,1^{2}, 0^{3}, 1^{2}, 0,1,2,0^{3}, 17,6,13$, $2,26,4,18,3,6,2^{2}, 0^{4}, 1^{2}, 2,0,2,0^{5}, 2,0^{4}, 2,0^{9}$

$\mathrm{A}(\mathbf{2 7}, \mathbf{1 0}, \mathbf{1 3}) \geq \mathbf{2 8 3}$

$0^{2}, 56,420,4213,616,3042,732,98.10921,2166,577.1260,0,299.606,249,4566,1544,579,478.0,143,145$ $150,224,361,38,431,267,241,0,34,2033,404,108,1062,0,1172,89,242,160,371,0,74.22^{2}, 17,129,101$, $105,31,14,64,14,31,27,12,0,67,3,33,0^{2}, 11,3,94,119,63,15,1^{2}, 6,40,75,69,30,0,45,31,7,93,71,24,41,14$, $98,40,7,6,2,3,2,5,2,18,14,2,39,22,1,3,0,11,3,0,1,2,0,2.3,1,6,0,2,0^{2}, 2,0^{5}, 3,62,0,23,150,5,6,79,29,8,6$, $72,8,13,2,12,62,29,42,0,4,0^{6}, 4,0,2,0,5,1^{2}, 0,9,7,1,2,0,2,4^{2}, 1,0^{2}, 2,7,0^{2}, 1,0^{17}, 43,14,13,20,7,0,1,9,14$, $19,10,4,0,10,0,3,1,0^{5}, 1,16,4,3,0^{5}, 6,0,4,1,0^{4}, 83,23,1,76,8,0^{3}, 46,0,10,1,0^{3}, 21,2,7.0^{3}, 3,2,0^{5}, 2,0,4,1$. $0^{2}, 2,0^{5}, 1,0^{12}$

\section{$\mathrm{A}(\mathbf{2 8 , 1 0 , 1 8}) \geq \mathbf{1 9 5}$}

$0^{3}, 56,286,1296,3684,5186,1441,138,0,833,5,115,4737,1382,956,86,373,96,51.2263,1799,21,135,242$. $103,342,104,49,48,113,75,17,8,2,1,667,1,269.55,58,19,35,365,86,2,92,15,37,4,21,0,11,0^{2}, 12,15,12$. $0,10,16,1,3,16,0,31,8,2,1^{2}, 9,0,1,0,2,5,1,2,0^{2}, 10,0,4,0^{3}, 2,4,0^{3}, 6,0^{4}, 1,0,1,0^{5}, 1,0,649,11643,515$. $44,27,21,20,33,258,58,39,14,49,30,3,7,6,21,31,49,29,30,2,9,1,8,0,22,23,1,6,13,7,93,5,55,10,6,1,0$, $10.3,0,2,1,2,0,1,0,16,0^{14}, 1^{2}, 0,9,0^{2}, 1,0^{12}$

$\mathrm{A}(28,10,11) \geq 280$

$52263,46674,39014,12594,7191,49805,257,1176,8505,2624,2904,1024,411,5583,9562,3356,214,1045$, $3611,2612,291,935,641,227,406,430,2926,3213,1039,2077,44,486,200,22,156,171,481,183,51,412,27$, $93,42,38,91,9,17,3,1410,278,244,96,100,17,33,132,24.10,6,31,106,11,7,4,15,74.11,55,6,3,22,4,8,0,1$, $0,29,0,10,6,11,0,1^{3} \cdot 0^{11}, 190,10,52,15,5,37,3,0,5,2,8,21,1,3,1,0^{2}, 15,0^{2}, 9,5,0^{4}, 2,0,5,0,1,0^{4}, 1,0^{36}, 47,3$, $1^{3} \cdot 2,5,1,0,1^{2}, 3,2,1,0,1,0^{2}, 2,0^{13}, 1,0^{5}, 1,0^{3}, 1^{2}, 0,11,4,5,0,3,1,0,3,0,1^{2}, 0^{2} \cdot 1,0^{2}, 1,0^{5}, 1,0,1,65,17,13,16$, $6.4,2,0^{4}, 3,5,0^{3}, 1,2,4,12,2,1,2,1,2,0,2,0^{5}, 1^{2}, 0^{8}$

$\mathrm{A}(28,10,12) \geq 356$

$0^{4}, 1212,865,887,143,95,906,134,674,11,56,2,0,44,58,634,6,0,39,157,8,47,14,8^{2}, 23,9^{2}, 50,31,11,0,3^{2}$, $5,17,0^{3}, 5,0,7,0,4,6,0.3,0,5,0^{6}, 1.0^{4}, 38,20,9,5,13,3,11,17,2,17,4,0,2,13,4,0^{2}, 3,0^{5}, 1^{2}, 0,2,11,2^{4}, 1,0^{3}, 2$ $0^{2}, 23,42,20,16,10,0,6,3,7,0,2,0^{2}, 13,3,0,1,0,6,0^{6}, 1^{2}, 3,0^{3}, 36,35,4,0,5,0,3^{2}, 4,0^{4}, 7,0,1,0^{6}, 8,11,6,7,1,4$, $3,1^{2}, 4,1,0,8,2^{2}, 0,1,0^{2}, 3,0,1,0^{6}, 23,8,2,1,4,2,3,0^{3}, 18,8,12,5,12,1,2,1,4,0,4,1^{2}, 0^{3}, 1^{2}, 0,1,2,0^{3}, 17,6,13$, $2,26,4.18,3,6,2^{2}, 0^{4} .1^{2}, 2,0,2,0^{5}, 2,0^{4}, 2,0^{4}, 6,1,0,10,7,4,3,1,2^{2}, 5,1,7,1,17,7,6,13,19,0,16,8,3^{2}, 0^{3}, 1,3$. $1,0^{2}, 1^{2}, 3,1,0^{3}, 1,2,0,87,16,64,31,6,28,23,3,2,18,9,16,7,8,1,0^{5}, 1,0^{2}, 2,1,4,0,6,1,3,0^{2}, 2,1^{2}, 0^{2}, 3,0,1,0^{17}$ $A(\mathbf{2 8 , 1 0 , 1 3 )} \geq \mathbf{4 1 4}$

$0^{2}, 56,420,4213,616,3042,732,98,10921,2166,577,1260,0,299,606,249,4566,1544,579,478,0,143,145$, $150,224,361,38,431,267,241,0,34,2033,404,108,1062,0,1172,89,242,160,371,0,74,22^{2}, 17,129,101$, $105,31,14,64,14,31,27,12,0,67,3,33,0^{2}, 11,3,94,119,63,15,1^{2}, 6,40,75,69,30,0,45,31,7,93,71,24,41,14$, $98,40,7,6,2,3,2,5,2,18,14,2,39,22,1,3,0,11.3,0,1,2,0,2,3,1,6,0,2,0^{2}, 2,0^{5}, 3,62,0,23,150,5,6,79,29,8,6$, $72,8,13,2,12,62,29,42,0,4,0^{6}, 4,0,2,0,5,1^{2}, 0,9,7,1,2,0,2,4^{2}, 1,0^{2}, 2,7,0^{2}, 1,0^{17}, 43,14,13,20,7,0,1,9,14$, $19,10,4,0,10,0,3,1,0^{5}, 1,16,4,3,0^{5}, 6,0,4,1,0^{4}, 83,23,1,76,8,0^{3}, 46,0,10,1,0^{3}, 21,2,7,0^{3}, 3,2,0^{5}, 2,0,4,1$, $0^{2}, 2,0^{5}, 1,0^{12}, 1,7,6,19,3,2,8,4,6,0,6,5,1,5,0,7,3,4,1,0,14,30,15,10,20,3,0,2,4^{2}, 7,0^{2}, 2,3,0,3,0^{2}, 1,4^{2} .0$, $2,1,0,5,0,1,0,1,0^{3}, 26,67,37,6,28,22,19,4,7,2,9.2,19.0,5,18,3,7,24,0^{2}, 8,1.0,1,0,1,0,9,0,2^{3}, 0^{2}, 6,1,3.0$, $2,1,0^{2}, 1,0,2,1,0^{2}, 2,1,0^{3}, 1,2,0^{5}, 1,0,1,0^{13}$

$\mathbf{A}(\mathbf{2 8}, \mathbf{1 0}, 14) \geq \mathbf{4 3 5}$

$0^{3}, 1150.96,5557,4719,2069,4941,1017,10572,1545.2879,3746,675,0,842,229,295,389,1949,163,2268$, $2130,9212,0,47,1563,161,174,210,429,0,218,1161.3037,254,700,65,4877,681,5297,706,0,330,820,60$, $34,411,436,1620,124,873,885,1639,503,513,0,408,643,160,1301,1860,396,5^{2}, 28,444,558,161,286$, $344.544,305,183,0,65.2416 .95,3964,1032,1720,38,105,851,58,1061,312,32,980,1003,187,198,0,60$, $508,221,249,117,53,187,28,107,1,424,210,12,460,305,176,6,4,0,214,19,0,8^{2}, 84,0,100,165,42,26,32$, $5,13,0,34,2,16,24,0,25,0.22,8,11,2,6,0^{3}, 2,4,1,0,130.398,240,208,257,40,52,232,106,6,2^{2}, 106,3,17$, $523.4 .215,115,17.20,6,142,3,1,12,93,1,0.8,4,71,43,15,2,24,5,13,16,26,0,3,1,0,4,2,13,1,0^{2}, 2,3,2,4,3$, $7,8,2,1,0,2,0,1,3,0^{3} .42,1,35,0,12,0,1^{2}, 2,0^{2}, 6,1^{2}, 2,0,2,1,2,1,0.1,0,10,0,1^{3}, 0,1^{3}, 0^{5}, 1,0,3,1,0^{3}, 1,0,1$, $0^{22}, 69,270,157,82,24,3,97,54,9,184,7,32,28,21,47,48,5,6,52,21,7,13,2,50,8,3,54,0^{2}, 31,2,11,67,2,21$. $42,3,2,11,1,9,1^{2}, 5,21,0,34,0,6,0^{2}, 2,0,2,1,0,11,6,2^{2}, 3,1,3^{2}, 0^{12}, 7.4,2,6,1^{3}, 3,0,1,3,4,8,0^{3}, 3,2,0^{2}, 2,7,2$. $0,1,2,0^{2}, 2,1^{3}, 0,2,0^{2}, 1,0^{5}, 2^{2}, 0,1,0^{7}, 1^{2}, 0,1,0^{19}$ 
$\pi(7,3)=(7,7,6,6,5,4)$, norml $=211: 32165426131543522314621534146242315$

$\pi(8,4)=(14,14,12,12,10,8)$, norm $=844$

5214334215143251362435261246153612442163516421625342631523415124334125

$\pi_{1}(9,4)=(18,18,18,18,16,15,15,8)$, norm $=2066$

17623428635817463454125177316422563873426148517253184325231283746465712458534721 6133574126187263635452417345612167327645741523

$\pi(10,3)=(13,13,13,13,13,13,13,13,13,3)$, norm $=1530$

594279368731456885696 A815924134132727981346526724153678986723131249257A794658148

$5932136745528129734872 A 96981635445986137$

$\pi_{2}(10,4)=(30,30,30,30,26,25,22,15,2)$, norm $=5614$

54712763112843623648312585431276459317626584345225436176184725341716326423357614 21487575321755814263362415413281825637142436751247573365365542824145671317242816 39246314182173853275647321562417418366342578654321

$\pi(10,5)=(36,36,34,34,29,29,27,27)$, norm $=8044$

28657343817173624561257134426131247583724512686231547863743812415158264512867245 38645273718164382552658738264312368411347415623164551244873216281724536372485163 72416563782738537214614567332718412585622361476418737325413364815225814354218471 312648782563

$\pi_{1}(11,3)=(17,17,17,17,17,17,17,16,16,14)$, norm $=2731$

7A4 53288175962311934912386465964A5768914257463279AA1372816496A7593475825382A12A1 586423157964 A27A8658571324358916A82413974536989A1581276279483786A34532714A691643 $79 A 25$

$\pi_{2}(11,3)=(17,17,17,17,17,17,17,17,16,12,1)$, norm $=2713$

5A13869754634872517937298A2483491561863279716289436A4157927415163A7286485627353A $8219748246325 B 93 A 18569712$ A 9254686 A 13954729156843 A $11 A 632 A 75498743985238475916857 A$ 64312

$\pi_{3}(11,3)=(17,17,17,17,17,17,17,17,17,10,2)$, norm $=2705$

25816741797459633968436 A $349852128571936255 A 2328741891746369782851454$ A 12217639478 9635817496 A 385612747 A 9163364 A $952925 B 78419583$ A 74236831256729 A 4A 5618931645978224 A 18573

$\pi_{1}(11,4)=(35,35,35,34,33,33,33,32,31,25,4)$, norm $=10724$

89546341268 B2 17762426598 A $84675 B A 7131$ A 59832B 319A46B37A545933761472218A9A291324175 $169568453 A 452893718693 A 42645742596832791$ A $815365163718 A 4227 A 375534938152949371426$ 842798 A6195856127654A393645782A3A81266A41571386742293745172979583619251A84A58341 5239637615497864812369285751368 AA 6742A47837629181932548492613A686184795732A52374 $917429138 \mathrm{~A}$

$\pi_{2}(11,4)=(35.35,35.35,35,33,33,32,28,21,8)$, norm $=10616$

A 951842513754 A3B3251A287473914656828946261735593672A9486145874B3921AB7517464329A 962389871528196338614 A 57973 A 8219576148 A2B324A 638 B 978431261775828 A $995642163758 B 43$ $1175243569 A 426133 A 8625428 A 361257513467598426945871763285694718 A A 3 B 62915437182913$ 9875442 B $159378276145825 A 6331947571282546364$ A25158634972615A445363452717298693A71 $8486719 \mathrm{A3} 32$

$\pi_{1}(11,5)=(66,66,60,60,54,45,44,40,26,1)$. norm $=25066$

93521728164179693243516743257448136274353299216532181547423817125643546365741675 25683212178596213428531647372912411395784842638345269411247539513641279275842586 14327215816738125637418268474354913212365681944526373712724418825616354873541462 32148535621771625945236881453412935878271537412329657214814563127359148724153326 48651385742923196815231822463941435764293146317587247635212164789931513289425643 67251524614 A36514533842842712371653458722135796185813721286934

$\pi_{2}(11,5)=(66,66,60,60,54,45,44,42,22,3)$, norm $=25046$ 63521827184179693243519843258446137264353287218532171548423718125743546365941695 $25673212187576213426531648382712411385794742637345268411249539513641289285 A 42576$ 1432821561793712573841728749435461321236569174452638361282441772561 A354683541482 32147535621881725745239661453412635676281538412329758214614573128357146824153326 $47651385742823196715231722463941435864293146317578248635212164978831513279425 A 43$ 
APPENDIX TABLE (Continued)

69251524714638514533742642812381653457822135896175713821276934

$\pi_{1}(12,4)=(51,51,51,51,51,46,45,44,44,34,27)$, norm $=22903$

864134 B31 9A 5564215638972 A89AA4 241791542 B 782763566978421634839 A51B2B 856396 B 5978A2 678235123BB 413872456596182A57683145471A91A2 4737694241A575231863B824152A89634B743 586A31513B972 94A59762115247179A38AB $94639512 B 432658 B 275754318$ A 82479236 A 5 A 36941825 3618B4 97A8 $1623415 A 377152948291434617$ B1 465978B3A5 3B9826938532BA9664 5B 182617926475 83A5782341532713269526B9A8781397845157481356278314B 469AA1425B2673941A519A3843527 686329469732B5AB97238461467593129A895187342846517A6418B69572593142493673A2516872 $5 A B 81417$ A 4325B9

$\pi_{2}(12,4)=(51,51,51,51,49,48,48,42,42,37,23,2)$, norm $=22843$

A234B 42B3B37A42659717886A 9851B65A19185698567959B 71A186B7392484321624375B898A1156 $17 A 68596873427136452279436274313248$ A $4255869 A 189156 B A 518 A 6187979562$ A $43829534437 A$ 2473A242B1336254815769736247432952431BA15861596782B6717199858A659865B1453A242B63 $312743421 A 3 A 4722754395168$ A2 $643725 A 34431$ A21 9A8 7B71 $8596412743632479432876891569 A 85$ 13A85167CB7168596B916599A781A58214353247743262A43527A3443162167A9541352427B33B21 495861 A8 95B673342763147225843596BA1 97B18AC61A8597493524A432612437679158586719BB6 79852A8A1596423

$\pi_{i}(12,4)=(51,51,51,51,49,48,48,42,42,40,15,7)$, norm $=22815$

BC41343A1136B 94758626897 A8952C75A28295789576858962B2B7A63A 4191342741365A989A2257 26A7B587963146237154468137461323419A13455978A2 982578A529A7296868574A1394B531136A 4163 A $41492337451 C 25678637416134$ A $541329 A 25872587694$ A $7626298 B 5$ 9B 75897582153 A $414 C 73$ 324613142A3A1644651385279A4713645A31132A 428A96B629587C24613734168134967982578A 95 2BA952763162795B7A827588A692A59421353416613474A13546A3113274276A8512354146833942 185972A 985 B 761314673216445913587BA286C2BA972A 9586383541 A1347241367682595976283C7 68954A9A258714B

$\pi_{+}(12,4)=(51,51,51,51,49,48,46,44,43,37,20,4)$, norm $=22795$

31AC2821CB233186579498B6A9A54865A4747568B569757A849476AA372181324621395B977A4456 4B96A58679312843615227813628134321891325596A 74A845687549764B9798562B1372A531139A 2193A212A43362518459679362181327521347A458645A698B768494B785A 9657B65A4 153A212763 342913124 A3A1 922851375468 B2 613925A311348248B79A8475B 61429136321A813279678456B875 4AA754692394685768746577BAA4957241353219813262813529B31134624697A514352129833824 1A576497A596B831296341A225813586C74B9748A3649A5712835217132642138698457576849296 $\mathrm{AB} 753 \mathrm{C} 7 \mathrm{~A} 4596 \mathrm{~B} 18$

$\pi_{5}(12,4)=(51,51,51,51,49,48,48,45,39,36,22,4)$, norm $=22755$

3C24B4C92327B43658717996ABA51965A181856BA567858971B186A72843932416432759A8891156 17A6B5A6872347126354479326473212439932455B6A81AB156B8519861A787956493284A523327B 43729434 A $1226453 B 157687264373248543218$ A 159615967 A 4867171 A895AB658A6591352A 434862 214732341 A2 $9374475328516 B 94632745 B 233219419 A 87 B 7185 A 6 C 14732624379324876891569$ A 85 12A851673B 7169586A816588A791B58413252437732464932547923321641678A531254347A22941 395861A8A5B672234762137445A32596A81A781B9C61B9587392543832461432767915858671A3B6 7A85498A1596B42

$\pi_{6}(12.4)=(51,51,51,51,49,48,48,45,41,32,22,6)$, norm $=22663$

A2 4C 34392B352946785159B6A9A71B67C181876AB765878951B186A52843932416432579B8891176 15A6C7A685234512637445932645321243993247796A819B176A8719861A 585A764B328495233259 4352943491226473 A $175685264353248743218 B 17$ A617965AC865151B8A7AB678A67913729434862 214532341929354457328716 A 94632547923321941 AB85C5187B62145326243593248568B176BA87 $13 A 87165945169786$ A 816788 A 591B78413272435532464932745B233216416589731274345A22941 397861A8A 7B654234562135447A32796A81B581A9261B9785392743832461432565917878651ABB6 5A $87398 \mathrm{~A} 17964 \mathrm{C} 2$

$\pi_{1}(12.5)=(80,80,80,80,72,70,69,67,67,62,48,17)$, norn $=55860$

965317A143671252437CB371B58274431A6998C15246383524931865165783A927244BA4953895B2 156A364A179A427312893B 4953895B1238AB976A487123B362414C99765235247762915A8B63135A 84782B983A65BA185672B61354A897221974283A481A7B6A7216135C3761525B3694CA21659612C4 5723484 AB8 15782614583 A 92B 84B6143798528319A667512A4865394AC1561B89731673254A1774A 8253B9B4C1A527BC4 156377A2 48724631985B98675CA1923A294B3415691565A7438B68372241642 
APPENDIX TABLE (Coninited)

A793164A527498312B972B1934A5A5863BA1 362438294598A815B 73547361242783A8311B9245839 26A718491457A8A261B5996C4217C69A253484BA267319A6581B72A6A3B9124851976813C2164B 43 $8772385693384 \mathrm{~B} 45621 \mathrm{AB} 7196524651884$ 5B 9A293764AB811398A624134852732761B16378413972 . 495A 95443972683 A25 4B7AC91CB82A633925 41A36B657429756AA46B1852392CB345741362785A 83 718B2 46916857389749346235921A421A357782A47619219AB393C586BA5842126B15749 $\pi_{2}(12,5)=(80,80,80,80,75,72,71,69,63,55,40,23,4)$, norm $=55350$ 9A5416712486135324B9A46185B37224167BA691532A474532861A851756A46838322B92854985C3 1579472 A 1689236413984 A2 854AC5 7134B7A8679286134C4732129896753453266739158BA741459 62963A584CA5891A5763871452C8963317923A49291B687A6317145C 4671535A47928A31758713B2 56342A28A6156B37125B4A93C82891246A5C3B418A77651372A9A4B2CC15A188B641764352816629 C354A 862D19536A 92157467932 B6327418B598B 76597183493B2A42157A15758624967B493321723 $9684172 B 53628 D$ 4136B 6371B 42D 595874A $914732483 A 2598 B 8159645264913236$ B 489411A9325946 3756152A1256898371A5887A2316C7853542A28537441B87CA166397A488132B51867614B31A2B24 A6634955A4 4B2B257319C8197532751BB25A8C3D 4 6728CB114889732142B53A4367191746A214963 2B5885224A $637949352769 C 819$ A 83C74483521C4787562 3A 6579627719834 A 39C42B621473695984 615A327B1795649B628427345831A2318456C93B2671A31A8C4A4B597895A23137C1562B $\pi_{1}(12.6)=(132,132,120,120,110,94,90,76,36,14)$, norm $=99952$ 31542766754122374813646275134216576238594 A1 5381925782131482843564917156629872265 $431786214546523 A 7123816493125445218367389185472361253487154215732837446328132588$ 144171278567253249634819 A 71562351245617322735348162485618563431277124381438 A2635 $1732747453261152546721345636198791632572315424 \mathrm{~A} 32712543898165826451246549153269 \mathrm{~A}$ 132581 A7843173425341612936543922361147725475564283169427256133871464215814279538 46123746272715345324151786786143352893124153351846226473427615516724374622648153 35142139825334168768715142354351727264732164835972418512464178331652724961382465 5745277411632293456392161435243713487 A185231A96235194564215462856189834521723942 45132752361978 A1 63654312764525116235474723715362 A 8341834217721343658165842618435 3722371654215326517 A 918436942352765872171441885231823644738237512451784352163274 5819837638125445213946183217 A 325645412687134562278926651719465348284131287529183 51A49583267561243157264631847322145766724513

$\pi_{2}(12,6)=(132.132,120.120,110,94,90.72 .42 .12)$, norm $=99776$

31542766754122374913646275134216576239594815381925782131482843564917156628872265 $431.786214546523 A 7123816493125445218367389185472361253497154215732837446328132588$ 144171279567253249634819 A 71562351245617322735348162485618563431277124381438A2635 17327474532611525467213456361 A $8791632572315424832712543899165826451246549153269 \mathrm{~A}$ $132581 A 7843173425341612936543922361147725475564283169427256133871464215814279538$ 46123746272715345324151786796143352883124153351846226473427615516724374622648153 35142138825334169768715142354351727264732164835972418512464178331652724961382465 5745277411632293456392161435243713487 A185231A96235194564215462856199834521723842 45132752361978 A 163654312764525116235474723715362 A 8341834217721343658165842618435 3722371654215326517 A 918436942352765972171441885231823644738237512451794352163274 5819837638125445213946183217 A 325645412687134562278826651719465348284131287529183 51849593267561243157264631947322145766724513

$\pi_{3}(12,6)=(132,132,120,110,110,97,91,75,47,10)$, norm $=99072$

32541677654211364923747165234127567139596825382415681232481843574926257718861175 $432687124547513 A 8213827693215445128376389285461372153496254125631836447318231588$ 244262169576153149734829 A6257135214572631163534827148572857343216621438243981735 $2631646453172251547612345737289692731561325414 \mathrm{~A} 31621543898265816452147549253179 \mathrm{~A}$ 231582A 6843263415342721937543911372246615965574183279416157233862874125824169538 47213647161625345318252687687243351843219253352847117463916725527614364711748253 3524123A815334278678625241354352616174631274835961428521474268332751616972381475 5645166922731193457391272435143623986 A285132A97135294574125471856289834512613491 65231651372969827375432167451522713546461362537189342834126612343758275841728435 3611362754125317526 A 928437941351675961262442885132813744638136521452694351273164 5829836738215465123947283126 A315745421786234571168817752629475348184232186519283 52849593176572143256174732946311245677614523 
APPENDIX TABLE (Continued)

$\pi_{1}(13,4)=(65,65,65,65,62,61,60,57,57,53,52,45,8)$, norm $=42165$ B326A54137863947341925B 4383296BD3A16124572A 89C9A 7B62 45C41952831C7674AB2C588C491B 349A721A75ABC85A67822164C9836A 7461352B197859A 34975CB95B11ACC 29482A6C32A612495B18 775883 B 9A3636751B5248CB914632D 68 658B 334 71591CA2 7469A361584522B 761ACB185A 4 18C769A 4 A 852 BC 1975834 B $183262 C B 35961 B 729 A 9368453 D 932 B 4735642$ A $132 A B C 86473796458157 B 34291 C$ BA574A12C816C24 9D83792B5A861CBC68A755A16759248453CA1 7B5B314A 963128576 B 913B54C 836 $212439 A 24$ 9D87B23718476828453949AB36C169427A 327C18578324BB261A64733B79816A28954 1 A5 796C9516AC2 3BC897B4116C6782C5523D25A69DA87514C79A3B451698281237C36B451A5346732 ADB $194463 C 18 B 748562516$ A $1237549346 C 7 C 836224 C 7 B A 182 B 597 A 3462534$ 9CB 7359BAB7123676AC 1482A 718C59C839214563B47B325A9476188124B3527639A 9C 8265716 A 9C1B58 4195A 42CB37 $\pi_{2}(13,4)=(65,65,65,65,62,61,60,58,55,54,52,45,8)$, norm $=42163$ B24 685312B962A3A23194573272496B827161435C487A 87CB 96435 C 31954 C216B6B 3A 94759 C $7391 \mathrm{C}$ 23A68419858C7A586B 944163 CA82CB7361254B177C56823C758A95B11B884937466824A614385917 B957A2 86D2926A51B54378B913624A67C57B223B15A18C4B3989261573544A9D1CCD175631B9B6CA 3A854CB17A592361724848725C61C8 4AA82B7352692473B256349124A68B73829C635A15C923491C B65739148A 16843BAA299475CA61D8B76C755C16D5C4373528B1975A213B9A2147568BA127538B27 41432A94 3A9C6B42B19396C47352A389B26816734CA2 4C8175A 924378461 B639229A8716B4CA5331 B5 9AC8C51AB $8426789 C A 3116768 B 475542 B 45869 A C 7 B 51398 A B 2 A 35178949142682 D 7351 A 5236824$ $\mathrm{ABC} 193362 \mathrm{~B} 1767385 \mathrm{~A} 4516 \mathrm{~B} 142 \mathrm{C} 53 \mathrm{~A} 23 \mathrm{C} 89 \mathrm{~B} 726443 \mathrm{~B} 6 \mathrm{~A} 71748596 \mathrm{C} 23 \mathrm{C} 45236 \mathrm{BA} 8257 \mathrm{~A} 97 \mathrm{C} 1426 \mathrm{~A} 68 \mathrm{~B}$ 1394A91785C89274135B2D37B24 58C3A617A1439254CA286C 974658167 A 81B593185634B 72D $\pi_{3}(13,4)=(65,65,65,65,62,60,60,58,57,54,49,47,8)$, norm $=42147$ B326A54137963D47341825C43932867B3A16124572AC8C8A786245941852931C7A74B62C599C481B 348A721A75ABC95A 67922164C8936A 7461352B187958A 34875CB85B1 1ACC284 92A 6C32A $612485 B 19$ 775993B8A3636751B524 9CB81 4632D69659B33471581CA27468A361594522B 761 ACB1 85A 419C768A 4A 952BC1875934B1 932D2CB35861B 728A 8 3C $94536832 B 4735642$ A $132 A B C 96473786459157 B 34281 C$ BA574A12C916C248C93782B5A961DCC69A755A16758249453BA17B5B314A863129576B813B54C936 212438 A2 48D97B2 3719476929453848 AB36C168427A32 7C19579324BB261A64733B78916A2985441 A5 786C8516AC2 3BC987B 4116C6792C5523B25A 68BA97514CA8A3B451689291237C36D451A5346732 ADB1 $84463 C 19 B 749562516$ A $237548346 C 7 C 936224 C 7 B A 192 B 587 A 34625348 C B 73589 A B 7123676$ AC 1492A 71 9C58C938214563B 47D 325A8476199124B3527638A8C 9265716 A8C1B594185A 42CB37 $\pi_{4}(13,4)=(65,65,65,65,62,60,60,58,57,56,53,37,12)$, norn $=42015$ A236B54127A6284724183594292386CC2916134573B98A8D7B6345C41A5392167674AD3B599B 481A 24AC731C75C98A5B67C33164A8C26A7461253B187958B24875B985A11CBA3849376A23C613485A19 B 75992B8C2626751B53498A814623D69659B224715C1BA374689261594533B 761A8A195C41AD76AB 4B853BA1875924A1923738B25C61B738A8269452BB2394725643A123ACB $96472786459157 C 24381 B$ DA574A138916C34 8AA2783C5A 961DBA6BB755C1675A349452CA1795B214A8621395769812B54A 926 313428B348BC7A32719476B39452848CA26A168437C237A19579234BB361C64722978D 16A3985441 A578CD 85169A 32B9C87A41169678395532A35C68AB97514B78D2B 45168A39132BA28B451A5246723 BDB18 $4462 C 19 B 749563516 B 1327548246 C 7 B 926334 C 7 D A 193 B 587 A 2463524 C B 87259 B 687132 A 76 D C$ 1493A 719C58A928314562B 47B235C8476199134A2537628C8C9365716A8C1B594185A436B27 $\pi_{5}(13,4)=(65,65,65,65,62,62,60,59,56,55,49,40,12)$, norm $=41975$ B235861429C52C1A214C 3671272395 A 827454316 A38 7B87CAB53169148639245A591CD 3765A71D 49 219C834D86897A6859C33451AC82B97154263A477B65821A7689B6944B883C17355823C543186A47 976A 9285D2C25964A 631 789A4 1B23C57567A2 21D46B48C3A1 9892A 4691633AB54 8BA4 76514BCA 5B9 18D 63B9 4 786C215472383A726B54C83B9B2A71625B2371C2651394237B597182C9516946AC213B4 9 A56A1C43874C831DAA2CB3769B54 98975D766845A6831A 1625A4C86C241BBD2 4376589A $427618 C 27$ 34312B931BC95A329481C5737162A1BA925845713CB23584767C23178354951A22C $9874593 B$ A 6114 A6CB58A64DB832978A9B1445758A376632A36C5AB97864198B92B1647893B432582A7164D621C823 B9B451152 947571 A 683645 C4 32A 61A2 198B8725331D5B7473B69CA21B 36215CA8267B9794325A58 9 41C38947A6B8B27341652917B236B918547C431A263C82A5A 9735684578 A 4B6914A6513B72C $\pi_{6}(13,4)=(65,65,65,65,62,61,61,59,58,54,50,32,18)$, norm $=41795$ C2 4765312ABC2 93A23194583282497B6271714359468B68 9CB7435A 31654821A97A3DB 495ABA3C1D 237A 641D65689B567AB4 41C39A627C9371254A18985A623AB568A5B11A6649384C7624C714365918 A958826BD2727B51A543868913724C78758B223A15D16D493B682715935449D 716CB185731ACD 78B 36A 54CB1A 95823D182464B925C71864B96278352AA2 483725734B124B9687362BA735815D9234A1C 
APPENDIX TABLE (Continued)

975 93A1 4 6817643D892AB4A5C88196D97A955617C564383526C1B65D2137BC21485768B129536827 $414328 B 43 B D A 97429183$ A 7C 4 9352B3A8B27617A34 9B2 4961C59824396471A73B228B6A17A4 895331 A5BC96C5178642D $968 B 8311797684$ A 5542B 45C 78AAB 6513968B2A 3517684A1429627B351B523762 4 ACB 18337291 A 8936574517 C $1429538237696 B 27443 C 9 A D 1 A 465 B 9823 A 452$ 3BAD 625C $97 C 9142 B 976 C$ 1384C91865A 682B 413572C3BD2 45B83971A814392549726A9A8475617BA61C583165A347B29 $\pi_{1}(13.5)=(123,123.121 .115 .110 .109 .109,102,99,92.84,72,28)$, norm $=135679$

1C4269A6943D 73559B1427352C6DA3BCA9172491189765318294673B8B6AD2B9A978371531473225 B $647 C 28 A 16 D 4 A C B 2174987 D 1162549$ C8A 65D 79B4 3BC5A2 47C 35185BAB376D 3289B1A587C62431294 CA91889B 63A 52CB 794215654 CA 33521A1768C172D 6184C3A62D 3152BA2867A43D 98BC57B83356765 2C 948 A 1294C 3BAD $7486213155 B A 4562821$ A 792B $39 A 776 C 89356642 B 81174 C 2 B 29837351 C 853 A 24$ B 6 511237596132C69A8C4A3997B 415BC8614235DB864981C535A762A1B 7827A 39C8416B 47158849219 634C625B58AD 3C612B 41A39965A789B 742 65A9CB2B 477216CC532194721DB 6327CAB 512C $3143 B 815$ 63A4 628429A8C8A7512699588438477A81526A733522C19C6B1494218455C9183695B6A226A8B319 C77514353CB 7463D1A2 6374186596A9748C192275314986A541 BCD76931739B3B2 465C14764A239B 68A571BD38A2365A9743451822879B1D59481B9265796843624761773A45313152942AA17376C531 9BB2C48 73A2C5D 741B2B $7185849261652 A 438346215378$ A 92B12A85C 47593281 B $164937 C 284563 A 4$ 812C85318C72753CABA66194387B6347611A92855B1364254AB26C8B4742C5ACB33C4D1751B 74662 5A4899A2561 8283B9192285B5364379837C1A 65361642278794C2A5B 749BCA5484363A58147D8C2A 9CB1863D23197B23746998A5BC8137163246AB7125619AB3762B4C2B3598613582631A4589142813 45CD457A7 98C917A 637258B1A 9D4D 642762A73C5C63214A8B 58775132139A 47219632A684C13CA 65 894B85513A8496A267749C73B491912B52436CA693B4275B134A1D 973865812 C2B $6337281 B 3754$ A 7 41 A 9521297433 ABD 43152 C $\cup A 25 C 5781694854$ B 76932713728CA9158A3A4687B694D1254A36591896 $5 \mathrm{C} 473 \mathrm{~B} 2$

$\pi_{2}(13,5)=(123,123,121,115,110,109,109,101,99,93,86,68,30)$, nurm $=135557$

1C4269A6943D73559B1427352C6DA3BCA9172491189765318294673B8B6AD2B9A978371531473225 B647C28A16D4ACB2 174987 D 1 162549C8A 65D79B4 3BC5A2 47B35185BAB376D 3289D1A587C62431294 AA91889B 63A 52CB 794215654 CA33521A17689172C6184C3A62D 3152BA2867A43D98BC57B 83356765 2C948A1294C 3BAC 7486213155 BA 4562821A792B39A776C89356642B81174C2B29837351C853A24B 6 5112375961 32C69A8D4A 3997B 4 15BC 86142 35DB8 64981C535A 762A1B7827A39C8416B47158849219 634C625B58AD 3C612B 41AD9965A789B 74265A9CB2B 477216CC5321947213B6327DBB512C 3143C 815 63A4 628429A8B8A75126D 9588438477 A81526A733522C19C6A1 4C 4218455A9183695B6A22 6A 8B 319 C775143539B 7463B1A26374186596A 9748 C 1922753149768541 BCD 76931D 39B 3C2 465B14764B2397 68A571BC38A2365A9743451822879D1B59481B9265796843624761773A45313152942AA17376C531 9BB2C 4873 A2D $5 C 74$ 1B2B 7185849261652 A 43834621537 AA $92 B 12 A 85 C 47593281 B 164937 C 284563 A 4$ 812 C 85318 C 72753 BABA66194387B 6347611 A 92855 B 1364254 AB2 6C 8 B 4742 C 5ACB 33C 4D 1751 B 74662 5A4 899A2 5618283B $9192285 B 5364379837$ C 1 A 65361642278794 C2A5B $749 B C A 5484363 A 58147 D 8 C 2 A$ 9CB 1863D23197B23746998A5BC8137163246AB7125619AB3762C4C2B3598613582631A4589142813 45DD457A 798C917A 637258B1A9B4D 642762 A 73C5C63214A8B 58775132139 A 47219632 A 684 C 13 CA 65 894B85513A8496A267749B 73B 491912C52436CA693C4275B134A1D 973865812 D2B6337281B 3754 A 7 41 A 9521297433 ABD 4315268 A 25C5B81694854B76932713728CA 9158 A 3A 4687B 694B1254A36591896 $5 \mathrm{C} 473 \mathrm{D} 2$

$\pi_{3}(13,5)=(123.122,121,114,110.109 .109,102,97,91.85,77,26,1)$, norm $=1354.37$

1C427A97A43B63559C1426352E78939ABC1624A118A6753182A 476388B79C2BA9C68361531463225 D746C28817A4ACB2164D86B1172549B8975C6B943AB5D246B351A5BDC36783287C195868724312A 4 BA9188D 7739539B6A4215754CB335219167CC1 629718A43972831529A287694 3BCBCA56B833576C5 2DA4C812C4A3A 9C648721315AB $94572 B 2186$ A2A398667BCB357742981164D2B2A8363519853924C7 511236587132 A 7B 5C 44938 CB $9415 A 8 C 7142359 D 8749$ C 1 A 585C672A1B 692693 AB $841794615 C 84 A 216$ C34B 72595B 9A3D 712C419CAA7596B8A64275AA 9D2B 966217C95321846213B 7326C99512B3143B815 B3B 4 72842AAC 7C $965127 C A 58843$ C 4669815279633522 B1CB7914A4218455BA1837A5A 7C 227C8731A 9665143539B 6473B19273641895C78A648B1C2265314987A541BAC67A31639D392475B14674C23AC 789561 D 938 C2 $3759 B 64345182286$ AD1B59 481AC2 756B7843724671663945313152842AA16367B531 C982C4A6392C5B64192861B5B4A271752843B347215368CA2B12985B465B32818174A36928457394 812985318C62653B9CD 771A4 386D 7346711 DD 2855B13742549B27A8B4642C59CB33C4A1651A 64772 5C4 8B9A2 5718283B91A228585374369A 36D1C7537174226B6B4C29AC64B9AA5 484373958146C8A2 9 BAC1873D23196B23647AB8D58BC1361732479B612871D9D36728 4C2B35587135827319458A142A13 
APPENDIX TABLE (Continued)

45CB45696A8CC16A736258B19AB43792672A 63C5C73214CAB5866513213D94621A73297845138C75 BA4A85513B8487B27664AD63D491912A52437BC793A4265A134B1A56387581292C73B6281C3654D6 41C 9521296433 AC $94315278 B 25 A 5681794$ A 54B67932613628B9C15893A4786B 784D12949375A1B97 $5 \mathrm{~A} 463 \mathrm{C} 2$

$\pi_{4}(13,5)=(123,123,123,116,110,109,106,100,98,92,81,68,38)$, norm $=134757$

B23968695A4 7B912864A516A4C937B4C1173823D315D16624C9A589281497A739574B313D1284D6B 8158A4D392745C67B38D28152C46285C9B7A52673B36149478161A48275B92A 73C3B162419826654 3365A59224778C451B3A61A7984498A3A571B325659A83C419671A6258641B72A 927358278179314 BAB2 376412594712A8A1365C8241359B629C7CD5278A431B785CA9631247B463A2947C162874591B 36C9AB2387653B65124B9D72AC8313B282465651327946ACD381A 984C7D2B7A1339653A 7859DBC 94 8651219BB32 47824A137B5816924526C38192DC4B571648B33D5A 6143A625B 758C3C645A19312492 A 178B21 5621DCC3B62 468B8776513A5292C 48759A37942C8B14A 9154C6229B283C8697546B1879DA 354C7213AD9B3 459176BA31246858B162AD6A4 95B2384637395C4D576871A92A 4189218C3647D526 31BC4D772A831D3591BA923465C64A87A53239BD 784219B7B56A2837144953369589162CB5234A 35 1768A 912B 756A1A14C19A 32546D7B8725B 4376C4D9C425824611B 72AD35876B4263897C138612145 67452C7A 185423 C 7983C732B1A8C827254358B63A1C96D 9466417 ACB $127535 B 7 A 3 D B 5146271288 D$ 3926436C9C5AC51B618243BC8A73496B57391A754816A935A92673341AB5129AAD 74D6819419726D C 315849285236 B 93C 841278673 AC 5189734 B 65A91C2C615B6A439C435A138B69D42B38165B692A 38 59D 41728C175235A 944132796928 A8547B1754375413263A51628247217D674 385A67B4A315B63D9 28B29C9314B63A8B9162C5C17583864D245A76751A4A3B9472313182568C6A5214735147A73A1282 9186CBD2331775921C64842CB13CBD54A 96AB9C2531498352CA17A324A52CB1B4C6897328B 569A 98 $1 \mathrm{~A} 35624$

$\pi_{5}(13,5)=(123,123,123,116,110,109,107,104,97,89,83,62,40,1)$, norm $=134753$

C 319686A5D478923864B526A49917C4B2271831A12592 6634A985893824B 7A719574A121B2384C69 A258D 4C1A3745A 67C18B38253A 46385A9B7C 53671C1624A478262C4D375CA3C 71B1926342BB 36654 1165B59334778A452A1D62A 7D844A8B1C572B13565A981C42867296358642D73BC37158378279124 A9B31764235A 4 723A8B216598342159963AD8CB53A7D 412C785DAB612347C461A3B4 7D263874592A 16DACB3187651965234BA973A 98121938346565213794 6DA $9182 B 584 A 793 B 7 A 211 B 651 C 79598 B D 64$ 885232A9A1347834921795826934536A182A 3DC4B572648A11B576241B6C5975DA1C64582A123493 B278C 325632 CAA1C 63468 B 8776521 B 5393948759 B 17643 A 8A2 4B 9254 C 633 A 8381 C8 8 B 7546 A $28799 B$ 15497321BA9D1 459276CB12346858B263BC69495D31B461719AA45576872893A428C328A1647A 536 129D4D773C812B1592AB831465964387A51319DB87432978C56A3817244A51169589263BA5314B15 27A8B 923B75692A24C2AB13546B7D 8735B4176B4A 9D4 35834622973CB15876C 4361897A218623245 67453A7C285431B79A1 9713C2B8B837354158561B2A 96C49466427BAC23751597A1 9B5246372388C 1B36416BAD5E952A628341CB8D71496A571928754826DA15B936711429D5239A 987486D2A4297368 C12D84C3B5316981CC 42378671 BD 52A 9814 A 65B92C3C625B69419A415D218C89B 43C1C2657693A 18 59B 42738C2753158944213796938B8547C275417B42136185263C3473278674 1B5C67A4B125D61A9 38D39C9124B61B8A9263D5B27581864D345B76752A4A1B947312128356AC6B5324715247971D23C3 C286DBC3112775932A 64843B6219DC54BA6BA9C35124981537A27B134653AB2C4D6897138D569AA8 $2 \mathrm{C} 15 \mathrm{D} 34$

$\pi_{1}(13,6)=(166,166,160,156,143,143,139,135,131,122,107,100,46,2)$, norn $=239106$

14B2365C352A 813897972 A 1 B 6493 B 27856617298514 BA3CA 784 A 3 $164753821 C 4 C 3 A 267554624$ B 619 971279A 4B22813636C1914579A69132B87C $91365 B 475549 A B 2 A 589 C 463713$ DC $95514863923 D 4 A 5 B 3$ 62481C97B2A81C6752D15882C913CA64BA9D311982AB774235273C843791A684259126C9A 45681DB 6199375A 68B2C 314CA3541D25A5817346AC26A 372B481C3D62512973648B1271A5B7CD8D51B2374C 59D 426C13AB 3A 786B534D 2568 CB 61395A 4 6D 37C84B51973A2446C1D7227A 73A482B56C187B23941 52B968A869421A6B3829411C5821C3725B4 96D357B9ED 356931A6472456AC1A4867356BC219D6459 8146154BA 89173CB422D3C 98473136712825 A6D23683C $89194 A B 1 A 2 B 354716$ A 7C $5482326591 C 78$ A 3 295B2856B6A734169A4B128A 937413259672 CB 451A6D 84A 7B1D57582345AD3C53B242A618174A 938 2B7CA32791864461B989A4A16B732B16575824B29716C4521349B 6DB4C1954A3752182753CC 97315 318AB 642A41298A 554623A67B137972A33681C94C275651D86B14B5CA329843B1B9665C8A 487 B 215 C536362A57298354 1CAA85382421713A4 97C915224751849829A38B92715A58C31429A 71C2681D5A 4A6536C81439287553712873AC4A63D96B41821C68BD38674 9CE57152A 783C14272165C5124738DB A 943C1826A681386AB97AC 98493 C1A2862331982563BC4 $628657549165979 B 432834278516$ A5277C B893113A4C41628BA6328795A73B949D82412A4B8B6539236AD4BC734571919248625A171C9C732D 
APPENDIX TABLE (Continued)

2B1C5437A67A6592C4B168524317A5381724CA984361D627B8ABD 649148A7A425152DB62756A8B 43 931491369A563BC9C17232976A 43C8143915257687DCB4 723B3D55C15B 318986748CAB 949127 A 782 3CA88162A 49492 CB 621 3D 58C 9273614 C67937BA342B 4168D257B52 6C1523A 98489684157 D 6543 A 95 - 4457 B 28321 C 6973916417 B253897DB6213A3AB2C8846B12A 983 A 43759 C 71B3C286593AC9B 6435 D 84 . 97CD 81742 B 5542A35842B31C59617946495797457163D 421B3826CAB3148675912385276A5C78624 31458B2 471 9D 216AC23B8 669714B 8114AC7322AC659543A 81 9D 3652C788B 3C64ACB 955327 B 41 BA 84 21369576156794 C 359732 C 7641 BC 54214 A 9718524 AB $32623869142973 A A 76232$ 4B11D $38 B 9 B 675384$ 3DC2135AB 7152A 884A5C3726C19B6819472A

$\pi_{2}(13,6)=(166,166,160,156,144,142.138,137.131,120,106,102,46,2)$, nonn=239082

14C2356B362A813897972A1B54 93C27865517298614CA3BA884A3154763821B4B3A257664524C519 971279A 4C22813535B1914679A59132C87B91356C4 76649AC2A 689B453713DB9661485392374A6C3 52481B97C2A81B5762D16882B913BA54CA9D 311982AD 774236273A843791A584269125B9A46581BC 5199376A58C2B31 4BA3641D26A6817345AB25A372C481B3D52612973548C1271A6C7BC8D61C2374B 69D 425B13 9D 3A 785C634D12658DC51396D 45A37B8 4C61 97BA2 4 45E1D7227A 73A4 82C65B187B23941 62C95858B9421A5C3829411B6821B3726C 495D 367B9CD365931A5472465AB1A4857375B 8219A5469 C1451C4DA89173BB42263B9C 473135712826A5D23583B89194AC1A2B 364715A 7C6482325691C78B 296C2865B5A734159A4A128BD37413269572BC461A5D84A791D67682346AB3863B242A518174A 938 2C7BA32791854451C989A4A1 5C732B156C6824C29715B 462134 9C5DE4B1964A3762182763AB 97316 318B 2542A 412 98D664523A57C137972A33581B94C276561D85914C6BA32894 3C1C9556B8A487C216 C635352A672983641BAA86382421713A 497BC16224561849829A38C93716A 68C3142CA71B258196A 4A5635B81 43D2876637128739B4A53DD5A41821B58C9385749B 967162D 783B14272156B 6124738DC A 943B1825A581385AC97AB 98493B1A2852331982653CB4528567649156979C 432834278615A6277B C 893113 A 4B 4 152CCA5328798A73C94BD82412A468C5639235AD4CB 734671919248526 A 171 C9B $732 D$ 2C1B6437A57A5692B4C158624317A6381724CA984351D527C8ACB549148A7A426162BC52765A 8C 43 931491359 A 653CB9B1 7232975A43B8143916267587DBC 4723C3D66B16C 318985748BAC 949127 A 782 3CA8 8152A 494 92BD 5213C 68B9273514B 57937CA3 42C 4158A267C625B1623A $98489584167 D 5643 A 96$ $4467 C 28321 B 5973 C 15417 D 263897 D C 5213 A 3 A C 2 B 8845612 A 983 A 437 C 9 B 71 A 3 B 285693 A B 9 D 5436 C 84$ 97BC81742D6642B 36842C31B69517945496797467153C421B3825BAC3148576912386275A6D78524 31468 B $24719 \mathrm{C} 215 \mathrm{AB} 23 \mathrm{C} 8559714 \mathrm{C} 8114$ AB $7322 \mathrm{AB} 569643 \mathrm{~A} 81$ 9C 3562D 788B 3D 54ADC $966327 \mathrm{C} 41 \mathrm{CA} 84$ 21359675165794 B 36A732B75 41C864214A9718624AC32523859142973AA752324C11C38B9B576384 3BD2136BA 7162A88496D3725B19C5819472A

$\pi_{3}(13,6)=(166,166,160,156,143,142,138,136,130,120,111,97,51) \cdot$ norm $=238832$

14 B2 365C352A81 3897972A1B 6493D 2785661729D514BA3CA7B4A3164753821C 483A267554624B619 971279A 4B22813636C1C14579869132B67D 91365B475549AB2A588C463713BC $95514863923 \mathrm{C} 4 \mathrm{~A} 5 \mathrm{~B} 3$ $62481897 \mathrm{~B} 2 \mathrm{AD} 186752615882 \mathrm{~B} 913 \mathrm{CAD} 4 \mathrm{BA} 9 \mathrm{C} 311982 \mathrm{AC} 774235273 \mathrm{~A} 843791$ A $684259126 \mathrm{C} 9 \mathrm{~A} 45681 \mathrm{DB}$ 6199375A68B2C314CA3541D25A5817346AC26A372B481C3D62512973648B1271A5B7CD8D51B2374C 59D 426C13 9B3A 78 6A534B12568CD61395D 46A 37C84B51973A24 46C1C7227A 73A 4 82B 56C 187 B 23941 52B 96868B9421A6D3829411C582183725B 496D 357B9DD 356931A6472456AD1A4867356BC219D 6459 8146154 BA 89173 CC 422 D3CA 8473136712825 A 6723683 C 89194 AB1A2B 354716 A 7C5 $482326591 C D 8 A 3$ 295B2856B6A734169A 4B12 8DC37413259672B9451A6C84A791D57582345AD 3C53B2 42A618174A 938 2B79A32791864461B989A4A16B732B16575824D29716C 4521349B6DB4C1954A3752182753AC 97315

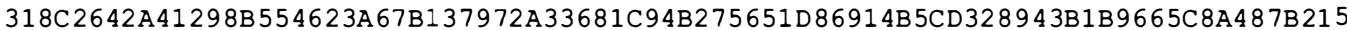
B 536362C572983941ACA85382421713A4957B1A22 465184C82BA38C9D71BA5B93142DA71C268195A 4A6536D 8143C287553712873954963AB6A41821C68B9386749C957152A783C14272165B5124738DB A943C1826A681386AB97AC98 493C1A2862331982563BC4628657549165979B 432834278516A5277D B 893113C4D 4 1628CAD 328795A73B94CD82412A 4B8B653923BAD 4BC 734571919248625A171B 99732C 2B1C5437A67A6592C4B168524317A 53C172 4CA984361DD27B8AB9C 46148A 7A4 25152CB62756A8B43 9314D1369A 563BC9C17232976A4 3C 8143915257687 DB 94723 B 3D 55C15B318986748CAB $949127 A 782$ 3CA88162A 49492 CB 6213 C58C 9273614 C 67937BA 342B 4168A257B52 6C1523A 98489684157 C 6543 A 95 4457 B2 8321C6973D16417B253897DB6213A3AB2C8846B12A983A 43759C71B3C2 86593AD 9D 6435 B 84 97BD 81742 C55 42A 35842 B 31C5 9617946495797457163 B 421A 3826CAB $314867591238527 B A 5 C 78624$ 31458B2 4719D 21 6AC23B8669714B8114AC7322AC65D54 3A81 9D 3652C788B3C64ACB 955327 B $41 B A 84$ 21369576156794 C $35 A 732 C 7641 B 854214$ A 9718524 AB $32623869142973 A A 762324$ B $11 B 3856 B 675384$ 3DC2135AB 7152A $88495 \mathrm{C} 3726 \mathrm{C} 19 \mathrm{~B} 6819472 \mathrm{~A}$

$\left.\pi_{+}(13,6)=(166,166,160), 156,145,142,139,136,131,118.113,91.50 .3\right)$, norm $=2.38698$ 
APPENDIX TABI.F (Continued)

1472356B36AC 71927AA82B1BC 493A2896551827C61 4DA3BA89473154B63921B4B3D258664524751A 78129AB 4822A1353591714687758132C58971356C48664D972E65BA4D3813CD76614B53723A4C 6A 3 524919B872CC1 95D62C169B28B13A9D4AB 773118B2B7A8 42362B3C $943 D 7195 A 4268125 C A C 46571 D 9$ 5 1'A 7386B5C8273149E3641A26B69183457825B3727491B3552612A 835497128146B8A97CA192384C 6AC 925813AC3BD 75863471265AD 951396 B 45 738CA4C61989B24 45C1D822B 983B 472C65A1A8723B 41 62D 95757A9421C5B392B411C6921C3B26A475736D97AD3657316548246598164A57385B9219B5467 A1451C48 97A183DB422 6377D 483135812996A 5723593B9A1B 48C192A364815B8764723256C18D 973 2A6A2B 657578341 A 9C4A127B9384132CA582CC46175DA 498B17686A2346253963B242B5171849D3A $29897325 A 1 A 544 C 17 B 89 D$ 4 715A832C15686924B278159462134A 75AE 471D64938621C29635978316 317B25 42A 4 12B9A664523958D 13B7827335917B4C28656179591496CB327A 4381 AB556A 9D 4 98C216 $7635352 \mathrm{C} 672793641 \mathrm{BC} 7 \mathrm{D} 63 \mathrm{~B} 242181394 \mathrm{~A} 8 \mathrm{DB} 1622456194 \mathrm{D} 927 \mathrm{~B} 3 \mathrm{AB} 9381 \mathrm{CC} 6 \mathrm{~A} 93142 \mathrm{CB} 8172 \mathrm{AB} 1 \mathrm{C} 6 \mathrm{~A}$ 4756359 A 1437289663 B 12C83764A53B 75D 4192185C7A35784AAB68162C893514282159B 6124839CB 7943318B565913752A78D5C94A381B2952331B926D 3BA4529568647156B8CA432734289615A 6258D 797311384 B 4152CC9B 32A 8CB 9837B 4C 7 A 2412946 ABC 63 A2 35CB 476 A 3468171724 B 52 6C1 819D 78325 2B186438758756C2D4C159624318B63A1824879A4 351C52AB9A9D54C14BD894261B2A952865A 9C43 B 314B1 357A 653B77A18232685A 43B 9143 A 16269578 CB 94823 C 3766 A1 6C31BA958 4 9DBA 74 C 1287892 3B9D7152C4B492C75213D6BC9283514658738C73427415AD268962571623A984A75B416895643AA6 4467 B2 9321B5A 83A 15418 C2 637B 8 9A5213939C268C 45612AC73A 438C7781B3A2C5673BDC75436D 94 78A971842B 9642A36842D3B71C517C454A6D 7846A1537421A39258B93149586C123B62879687A524 3146D72 4817A215B8239D 5578147C114998322AB56D 643C91BA 3562C8BAB3954DC7A66328741A7C4 $2135 C 785165 B C 4 D 36 B 732 A 85419 B 64214$ B $981 B 624 A 732523 C 5 A 1429 D 38 A 852324711 C 39 B 5 C 5763 B 4$ 3782136BA8162D9A4967382571CB571A4829

$\pi_{5}(13,6)=(166,166,160.156 .145 .142 .139 .136 .131 .119 .112 .88,52,4)$, norm $=238384$

$1472356 C 36 A D 71927 A A 82 D 1 C B 493 B 2896551827$ C61 4DA3BA8 9473154 B $63921 B 4 B 3 C 258664524751 \mathrm{~A}$ 78129AB4822A1353591714687758132D589A1356E48664C972C65BA4D3813DC76614B53723A4D 6A 3 524919B8 72DC1 95C62D169B28B13A9C4AB773118B2B7A842362B3C $943 \mathrm{C} 7195 \mathrm{~A} 4268125 \mathrm{CAB} 46571 \mathrm{C} 9$ 51A 738DB5C8273149B3641A26B69183457825B3727491B3552 612A835497128146C8A97CA192384D 6A6925813AC3BC75863471265AD 951396 B 45738CA 4C 61989B2 445D1D822B983B 472C65A1A8723B 1 62D95757A9421D5B392B411C6921C3B26A475736D97AC36573165482465981D4A57385B9219B5467 A 145164897 A $183 C B 422 A 377 B 483135812996$ A $5723593 B 9 A 1 B 48 C 192$ A $364815 B 8764723256 D 18 C 973$ 2A6A2B 657578341A 964A127B93841326A582DC 46175B 7498D1C686A2346253963C242B5171849C 37 $29897325 A 1 A 544$ B 1 7C89B4 715A832C15686924A2 78159462134B75AE 471D 64938621629C 35978316 317B2542A 412B9A66452395CD 13B7827335917B4D28656179691496CB32754381AD556A9B 498C216 7635352C872793641AC7B63D242181394A8CD16224561947927B3AB9381CC6A93142CB8172AB1C6A 4A56359A1 437289663D12C83764A53B75B4192185C7A35784AAB68162C893514282159C6124839CB 7943318C565913752A 78C5A 94A381C2952331B926C3BA4529568647156B8BA432734289615A6258C 797311384 B 41 52CD9B 3258CB9837B4C7A2 412946ABC63A235CD476A 3468171724B52 6D 1819B 78325 2B186438758756C2D 4C159624318B63A1824879A 4351C52AB9A9D54C14BD894261B2A 952865A 9D 43 B314B1357A 653B 77A $18232685 A 43 B 9143 A 16269578$ CD $94823 A 3766$ D 16C31BD 9584 9DBA 74 C 1287892 3B9D7152C4B4 92C75213D6BC9283514658738C73427415AD268962571623A 984A 75B416895643AA6 4467 B2 9321B5A83A15418C2637B89A5213939B268C45612AD73A438D 7781B3C2A 5673BDB 75436C 94 78A971B42C9642A 36842B3D 71C517C454A6D 7846A1537421A 39258A93149586C123B62879687AB2 4 $3146 \mathrm{C} 724817 \mathrm{~A} 215 \mathrm{~B} 8239 \mathrm{E} 5578147 \mathrm{E} 114998322 \mathrm{AB} 56 \mathrm{C} 64$ 3D $91 \mathrm{DA} 3562 \mathrm{C} 8 \mathrm{BAB} 3954 \mathrm{DC} 7 \mathrm{~A} 6 \mathrm{~B} 328741 \mathrm{~A} 7 \mathrm{C} 4$ 2135A785165BD4C35B 732A8C4 19B64214B981B62 4A73252385A1429B38A852324711639B5C5763B4 3782136BA8162D9A4967382571CB571A4829

$\pi \pi_{6}(13,6)=(166,166,160,156,145,144,137,132,127,118,111,98,56)$, norm $=238116$ $14 \mathrm{D} 23599368 \mathrm{CB} 182 \mathrm{~A} 747281 \mathrm{BA} 4 \mathrm{C} 3 \mathrm{~B} 27 \mathrm{C} 659172 \mathrm{AB} 614 \mathrm{D} 93 \mathrm{~A} 87 \mathrm{C} 4 \mathrm{~A} 3154 \mathrm{BA} 3 \mathrm{~A} 21 \mathrm{D} 9 \mathrm{~A} 34257664 \mathrm{~B} 28951 \mathrm{D}$ C7125A84722713C3591A1 4678837142C57D $91356 C 47664$ B 982B658A45371398A6614D53A23B4A653 52491C78B2A81C5D62B167B25A1379C4B8A8311C82A 97942362936843 CA1B584276125A7A465B1DC 518A37D95AB2 9314AC364182DB8D17345A62593A264C18355261277354C812D1968C97D891C2374B 67B425713AC3B685C634812659DC513B6D 45937D54A61973B2445A18C22CB7394D2B658187C23A 41 628B5C57BA4 21C59382941186721C382674A5A369B788365931654B246598184C59373BC21965478 81451C4ADA71739D 422834965731357129A6C5D235738C91847B152B364915D7A64A2325671A8B93 29692B65 45A7341C965812AB83741A26B5D2C346185DC488B1A6768234B253963B24285191A49B3C 2B7A9325C17A4491688B74 7159C3291A68692492A519C482134C75C94A1A64837621627D35A97316 
APPENDIX TABLE (Continued)

31BB25 426412CD8664923A56813BCC2933B7168482B 7561A96714B658329543719655CAA84CB 8217 963539287A2B736419AA56342421B13CA8C 981622486174 B928C37A9A71D75993142C8B1D2691B68 4D5635C7143D279A 63712C83B64B53CA5641821A579B359746CA67162B783514242158A6124739AD BA93314B5C5D13B52967A59848371A2B52331D82673B5452856764915677C9432B342C8615B62579 A 9C3113B 4A 61C2AB 983257AB1 83AB4C952412A 46C9CB37235ABD 867346B1918249526D 1718AA732D 2D1964371C7A5 6A2B49859624318A63917248AA8 43519527DCCBD 5 4814597C42A1A2B 8527659 BD 43 8314D135A 965386CD1 723267594 3DC143C1826759768C4723D3166CB6D 31B98574C899A4A12 7B8A2 39ABB 15284A 4A2795213BD9B5273514657837B6342A 418C9267BC25A16239AC4BA584167B5643986 $4467928321 \mathrm{C} 5 \mathrm{~A} 73 \mathrm{~B} 154179263 \mathrm{AB} 7 \mathrm{~A} 1521386 \mathrm{BA} 297948612 \mathrm{C} 983 \mathrm{~A} 437 \mathrm{CAD} 71 \mathrm{~B} 392 \mathrm{C} 5 \mathrm{~A} 637 \mathrm{BC} 954368 \mathrm{~B} 4$ C7C 85174296642836742 D 31C69517B 45 486A874 6D 153B 421 83C2BD8B 3148576A123A62 79C8979824 3D 469C2471C6215B923B6C58714BD114A6C322A85986435B15B3562A 7B8C39547C8969327D418674 2638BA75195CC 483BA732D 754189642149CD19A24A832523BAD 142 C 538B75232461163B8565793B 4 3BA21358971628B6485A3725B1DCC91A4728

$\pi_{7}(13,6)=(166,166.160,156,145.142 .140,136,131,118.106 .86,59.5)$, norm $=237556$

$1482356 \mathrm{D} 36 \mathrm{AC} 813287472 \mathrm{~A} 1 \mathrm{~A} 94 \mathrm{C} 3 \mathrm{~A} 27 \mathrm{C} 6551728 \mathrm{~B} 614 \mathrm{AC} 3$ 9D $79483154763 \mathrm{D} 2194 \mathrm{~A} 3 \mathrm{~B} 257 \mathrm{~B} 64529851 \mathrm{C}$ 8712 CAB $4722913535 \mathrm{C} 1814678857132 \mathrm{C} 97981356 \mathrm{~B} 47664$ A 982 A 65B 94 C $37138 \mathrm{D} 96614853 \mathrm{C} 23 \mathrm{~A} 4 \mathrm{~A} 6 \mathrm{~B} 3$ 524919A782DB1 95762C169B27B13AD54AB88311792C8A742362C36B4378195942D9125CEA4 65B1A 9 51D837CA5B9283148D3641B26A69173458725B3828491C3552612A7354981271D6B7DB8AC192374A 6B6425A139C3C785763481265CA9513A6D 45837DA4B61B73A2445B1B722A973A482D65B197823A41 62D859C85A 421A5B 392B $117692193 C 26 B 485836 C 98 B C 36583165472465 B 71 B 49583759 D 21 A 65478$ C145164C89B173AD 422B388C473135712996D5C2359389A1A47B192A364715B 7864923256B1D7983 2A6C2 96585873415964 A 128 BA3 741326 A 572CD $46185 C 8497$ B 1 A 6769234 B2 53963C24275181A 48B $3 B$ 297D8325A1954 4816AD 984A1 5C732B15676924B287158462134A759A481764937621627C35987316 31BA25429412ABA664523957D13B872833D918A492765618C6B14C65A32A54371AB556A9C497C 216 86353528B 728C3641CA8A 6342421713BA979816224A61548928C39D8EB1A96DB 31429C71A2 6A1867 49563597143B298C637129B3864A53DA5B41921C5A 8D 35874AAD 6716297C3514272159A6124739BD 8943B16A5C5B13852A87859B4D3A1B2952331CD26A3B9452D567648156B7AC432834279615A6277C 8983113D4B4152BC8D3257BC9738C4A8C2412946A 9B639235AB 486A34671C1824C526B1719D87325 2B17643785785692A4D159624317B63A1724C89A4351C527B9D9A54C14CA 79426162D 952765ABC43 D 314A1358A653D88C17232875A43C $9143916267587 B C 9472393866$ A 16B31BC9574 9ABB84D 1278792 3C9A8152D $49492 \mathrm{D} 85213 \mathrm{~A} 6 \mathrm{~B} 9 \mathrm{C} 273514657837 \mathrm{~B} 83428415 \mathrm{~A} 9267 \mathrm{~B} 6258162$ 3AC7 $498594167 \mathrm{~B} 5643 \mathrm{AA} 6$ $4468 C 29321 A 5 B 73 C 15417 D 2638 D 7 A 9521393 B C 267 B 456129 B 839437 C 8871$ A3C2A 56837BBC5436A 94 879C81742A6642B36742C3B81A517B 454A698746A1538421B3C25DA93149576A123B6278E6B89824 3146BA2 471AE2159723AC55D 71487114 9DC322A856A643B91 A $93562 D 7 B 983 D 54$ CD 896B327841A 874 2135B 875165AC4D35C732B7D41BA64214B9D1B624D832523D591428C3DA752324811639B5657B3C4 39C21368B 7162C9D 486E3C2581B 75B1A4729

$\pi_{1}(14.4)=(91,91,91,91,81,79,78,77,74.73,71,62,42)$, norm $=79393$

61B8473919C812581BA53DA7141B2976148C74235A9D3A6586B 472A2736BC1 9B25C94342A 7 C $7349 B$ 156C4235D 762C835B28CA83699B13CD2B417385CC4A 9612A397A6C8DB4A6582D 7B9517424396C263 55D82168217143D63D 48CB3659B1542389CAl 1ABD7 4A674925A8134672592347C6D3B 4 7C95649C23 8A5B762BA 45718CB81354561A2592DA8A8164BA1231D74C1732D9B18D4273A 515629C6B3A81 93CB 4 A 77C 4 5B328597C24B612823574A 463392456DA5BB6CD2 4391A8B 675C1A29A71348ACDB83179A6219 B7B4135D 56784261483595343CB1828791A7B2CD 56418267C3CB13BD89A7957A1148263524936C23 54C89AA7962451D9428AB2 7365ADBA3861CC643D928747325DB16C5764D83241B918A5673B16491C 52C 7844512 AB 582B 3A 78735 CA 194291543 A 6C1B8 4 7B3AC2B2 97863134591 B5B 3A 17829 B2AC 148 A 96 2D3C $963758424186 B 95 A 1 D 7 A 213 D 894 B 9655938 C 1235417 D 84 A C 2369 C A 6 B 7248568254$ C 7B133CDB8 293A7 6B2 48A 5C36D 4592374653781B184531A5C42B791B6278AB1A71 6D 6529D43859741242987634 $971646189731248 \mathrm{CAB} 2$ 9C95A381283CA 56B958672124BC1 95A231 8D63CAD 64984B937A5B 5637D 21 7A6249C318195B 761DA9 498B62353C254A8152C7B63494A3152B 7682C189547A1D46721AB63C7D 32 954638 AB1DC3B2D 5A764A67483512CB543682A719 $\pi_{2}(14.4)=(91,91,91,91,80,79,78,78,75,74,71,60,41,1)$, norm $=79357$

61B84 72A1AC813581B952D97141B3A76148C743259AD296586B 473937268 C1AB35CA 42 4397D 724AB 156C4329D763CD25B 38C982 6AAB12CD3B 417285C849A61392A796D8CB4 96583D7BA5174342A6C362 95D 83168317142 C 62945 DB2 65AB 154328 AC 911 DB8 749674 A 3C 981246735 A 324 7C6D2B 47CA5 $64 A C 32$ 895B 763B 945718 CB8 1254561935 A 3B 9898164 B 91321 D 74C1723DAB1 8D4372951563AC6B2 981A2CB4 
977C45B2385A7C34B61383257494622A3456C95BB6C9342A198B675C193A9712489CDE8217A 9631A B7B4125D567843614825A5242CB18387A1D7B3DC5641836792CB12B98AD7A57D1148362534A26C32 54C8A 997A63451DA4389B372659DB92861DC642BA38 747235CB16C5764D82341BA1895672B164A1C 53C78445139B583B2978725C91A 43A $154296 C 1 B 847 B 29 D 3 B 3 A 78621245 A 1 B 5 B 291783 A B 39 C 1489 A 6$ 3C2CA 62758434186BA591D79312C8A4BA655A28D1325417B849C326AC96B734856835497D122CDB8 3A2976B 34895C26D45A3274652781B18452195C43B7A1B63789B19716C653A54285A741343A 87624 A7164618A721348C9B35CA59281382C9B6DA58673134BC1A593218D62C9D64A84BA2795B5627D431 79634AD 2181A5B 761DCA 4A8B63252C35498153C7B624A492153B7683C18A54791D467319B62A 7D23 A546289B1DC2B3D59764967482513CB542683971A

$\pi_{3}(14.4)=(91,91,91,91,81,79,79,77,76.71,67,67.38 .2)$, norm $=79399$

71C9462818C913591BA52CDE1 41B3867149C64325E8B2A7597B 463A36279C18B35C842435696248B 1D7C43258673DA28B39C8927A8B12CA3A416295DC45B713D286B7A9CB4A7593D6B8516434287C372 55A9317931D142672A456B2758B1C43298CA116A9D4D764538A9124763583246C7D2B46C85748D 32 9A5B673BA45C196B 91254571 A 3583DA9AC174BA1321B64C1623B8D 19C 4362A515738C7B2A 9182DB 4 AC6D 458239586C34A713932564A 4722834579B56B7DC342D1A9B765C1A38A61249AC8B9216DA7318 B6C41259576C $4371492585242 \mathrm{CB} 1939681$ A6B 3CA57419376B2CB12BA68CD85691149372534827B 32 549D8AA6873451CD439A836275A8BA2971DC7426D396462 35CB17C5674D92341B819A5762B174D1C 53C $69445138 B 59382$ A 69625CA184 3D1542A 7C1B946B2C83B386972124581B5B2A16938B3AC 149 A 87 3C2C 872659434197 B 85A 196A 312CB8 4BC755829A1325416C84AC327BCA7B6349579354A6D1225ABD 382A 67B349C5C27B4583264752691B194B21A5C43B681D7369AC1A6178753AD42958641343896724 861747198621349 CDB38C85A291392CA5 7B859763134BC185A3219C72DAC74894C826A5B5726D 431 6D7348C219185B671CA8489B73252B354C9153A6B72484A2153D6793C198546A1847631A872D 6C23 854729 AB1CD2B3D5A674A76492513CB542793A618

$\pi_{4}(14,4)=(91,91,91,91,82.79 .78,77,75,72,67.62,45)$. norm $=79269$

638C49283B 7C315B3A55279A343617CC348794125BA62865BCA49171927DB3CD 15B742415B7924C7 3C764125AD618B2D 8165AD29BCB32891A4372856D4A6731B2C9DA67864BA5D1869A53841429B 7182 55C913771353426D 284A 69285D 635412BA 9C339B7A467D4815DA3246815D12496782947C9584AD 12 6A5B 7B1C64593A8A73254583B15A1 7D8D93B48631237A4C3621CB93B 741A2C535918BD62C8382694 C79B 45621A5CBD 149531 A12DB464822814677B5C98BA142835C8769C391A6A324DB6A9723DB65138 CC54327A97B541D34725A5242CA3717D9369618C5B43C19D82893296B67A 75DC334A192514726C12 54896D6A8B14537C419D8172A5D8CA297369742B818B4B215A63A876B 47C21438A3C 956826354635 819B744731989D1A2CB79258B36418354265A37C4C829A1A179B623245B3C5B293DA1C81673478BD 192CA 9275D 41436 A 79583AB6132ACB 467955C2D6312543B8748C12A6B96A8 1475D 71548BA32257AA 152B97A1 4865D2C84C612D4A725D 36384D2368C416B93B 7189CD39A3CC681 8B 4297A54314167BA24 6839483BAC2 3149B 751AAD5B2731C2875699568713146B385A123DC92A 9C74664B9278575C296413 6C7148923D3957BA 36B847CA612528154693519C6824A4B235178CD1B39D5496364CB1369A27CB21 A 5452A6D3C82A1759864AC7482531B85427A19B36

$\pi_{1}(14.5)=(169.169 .165 .156 .156 .152 .149 .144 .143 .137 .1 .34 .121 .118 .80 .9)$, nerm $=291280$

864C73E574DB519391AB2 3D415CE4B1A7B989A 7CC34E2B68AD132D6CAC6521 9B8A 358D $219448 B 35$ ADE 62AC62747B29568714453327DB9A3AD 571BE2998B659126BAB828C695A4C276AC $189978345 B 73$ 656A8A9E 3727245AEE2F11E2B3D8B75451793841BD9AC7297736142B5CE 6939352A14DCD1C95B 463 D6EDC87A 98121EBA7382528A64B72B616289EC54 93DD8C5275D873DE9A6816C95425C16844A81372 AEB 37CD 41283ED2 4 1A 53E9918ED5A6DB7538169AA4812BCBC1D78B7234853492DC386E 9AD 14157AD 371 A3514126BA3B742664AB8B39C.9C5E2D 46516D7AC538B26B72C892143956DA19C7A5E62B37469A 3B17D 481326874A 51B5623492B7215C4A58D67588D996C3A 94B7543727DB3634C16D9182ED172B59 ECB 495D8C4DA5A71E7D2832C3481C3754825A3D6321B 791829A461C1B75BC92 9311C4FD5 9B6734D4 62A87B6CB235418A 54E187A63E38629596CA7384515B17281E6DD453B2239686A 4B486913BC28E39 5A12B 75C $123594845 A A B 73 C C A 617235 D B F 69 E 1 B D 7 E A 672 C 4 D 636985 F 758 C A 12392 A 8 D 1475 B 3 C 9213$ 45684251D82E235A14A 7C6E1 9D86C91 7826839ED756361D84B245D597B2C9A4 7638A614B67BAC227 43C3A48771C6531BE2B5E6D 937281A4C19834D7A16C34E1D3B 1697 AB 9CB 659E $442838 C 2 A 54 F 3 D B C 2$ 9B4185275DA16395DB286E194531D24B5A6372A5BCA4E 86159D 47C65A9BC3139C153C4A221B 76B39 83251DAD 47B87E6CB5587DB139271D56A 62495C48C562A14 22C587E69EA32E67D94A517368C394B7 1D324B951762B9AD 318D7AAC9B166849758231A7398C4654C3E2E 32A16D1295A4A38C8 67B8 65D2A6 B91D5752884913ABE 74563FECD172DF8ABA1C37294864AE7D2C3392C55718ED2D 56714 7CB2 143EBA 314829627A3483A1B5218934C82D6615E4C82B758D48D7621536D 762C58437B659E219A85394BD51 
APPENDIX TABLE (Continued)

69B3DEC769E4346A5A57B3DC8171B29318B4DA3ADBC5E12E24C67615A3E8891435C646C72819D565 42A2ADE 12 CD 74 3D54C91BDA378FE6C723792164BD18946A1B5A85273D5D 434C9E6219BBE6371A68A 5719EB3A97C4 91C35EAB5F 42D8 6243 B $7183492 B 65712$ AC78D 1942673264957 A1687123DD325BCB 42 -68E69187A 598 AE 43C17B98 65CDA9D 4 452A 3CB52B1A4EC32C7136CE3A6172B 43A8914175D 269A2181 -B2 952BA73DC64 8B9 6354AA4 792CBE 961 8A 75 735B84 6B2 83C9D4 35A62 137284551476C312B8D9A5DE A2 9B6471C3DE82 563C914B967D14C258C1397CDABE7E 43C98B746851235A12BBDE9AB2 35C61D 3EB2 1895529 A 3E8D1 9562D4D 43786BEDB4 329657526B8A138C98C751421A 463C792BEC1A736B 854294D2 61367842C9A6D3CB 8134C9A15B248658337D121E64D2A 75B954CA 78E3746912C91A236E 81B9D8AC3 57

$\pi_{2}(14,5)=(169,169,165,156,155,153,151,147,143,137,134,120,112,76,15)$, norm $=290646$

964D72E57 4CB51 82 81AB32C415DC4B1A7B8 98A76D24C3B69EE123A6DAE65318B9A259E1318449B25 ACE 63AD 63747 B 38569714452237 CBDA2AC 571 BE 3889B658136BAB939D 685A 4D 376AD 1988C $9245 B 72$ 656A9A8E2737345E8E2B1 1E3B2C9B75 451782941BC8AD 7387726143B5DE6828253A 14CDC1D85B 462 C68AD97A89131DBAE293539A64B73CB1639A7D54 82EC8D5375C972FD8A6916D85425D16844C 91273 AEB2 7DC41 392ED 341 A528C81 9EC5A6CB7529168AA4 91 3BEBC1879B 7324952483CC29678EA14157AB 271A2514136BA2C7D36 64AB9B2 4D8D5E3C 46516C7AE529B36C73D983142856CA18D 7A5E 63B27468A 2C17D491236974D51B5632483B7315D4A59C67599E886D2A84B7542737CB2624D16C8193EC173B58 ECB 485A9E 4CA5A71D7E 3923D2 491D2754935A2C6231B781938A461E1B 75BD 838211C4FD 58B6724C 4 63A 97B6EB325419A54E197A62D2963C586EA729 4515B17391C68B452D3328696A 4B496812BE39C28 5A13B75D132584945AAB 72DDA617325DBC68E1BB7DA673D 4C626895C 759EA1328 3A 9B 1475B2C7312 45694351 C $938325 \mathrm{D} 14 \mathrm{~A} 7 \mathrm{D} 6 \mathrm{C} 1 \mathrm{CE} 96 \mathrm{D} 817936928 \mathrm{EC} 756261 \mathrm{C} 94 \mathrm{~B} 345 \mathrm{~A} 587 \mathrm{C} 3 \mathrm{D} 84476298614 \mathrm{~B} 67 \mathrm{BAC} 337$ 42D2A49771D6521B83B5E6DD2739184E1892CA7A16C2 4B1D2B1687AB8EB658E44392 9D3A54D2CBE3 8C4 $195375 \mathrm{CA} 1628 \mathrm{ACD} 396 \mathrm{E} 18452$ 1C34B5A 6273A5BDA 4B 9615E $847 \mathrm{C} 65 \mathrm{~B} 8 \mathrm{BD} 212$ 28D152D 4A331B $76 \mathrm{~B} 28$ 92351DAD 47E97A6DB5597CA12B3718E6A63485D 49F562E 1433D597B68CA23E67C84A517269B284D 7 1C234BD 51763B8AC2 19E 7AAD 8E166948759321A7289C4654D2F 3B23C16C1385A4A2 9D 967B965D 3C 6 BC1 85763994812 ACE 74562 FCBD 1 73BD9AE 51D 27384964 AE 783C22F 3D 5571 9E 83C567147DB31 42ACA 214938637A2 492C1B5319824F93D6615E4D93A759C 49D 7631526F 763A59427B658C318A95284BC51 68B2ECD7A864246E5A5 7B2CC 9171 B 3821 9B4EA2A8BD5C13E34D67615D2E 9981425 A 64 6D $72918 C 565$ 43A3ABE 13BC7 42C54D81 9BA2 7DFE6E 732783164BC1984691B5A95372C5C424C8D6318BB86271A69A 5718DB2AC7D 4 81D25EAB5C43A96342B7192483B65713A579F1843672364E57A1697132EC235BDB4 3 69E 68197A 58DAC 42B17B8 965CEA8F 4 453A2DB53B1A 4BF 23D 7126EC2A6173942CE814175C368A3191 B3853BA 72CD 64 9B86254AA4 783CDC8619A75725D946B392D8C425A63127394551476D213E9C8A5DA F 38B6471D2CB 93562F81 4E867A1 4D359C1287BD8A67B42B89A746951325913CBCE8AB325D61A2EB3

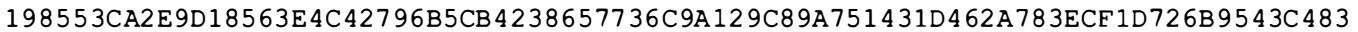
61267B4 38CD6A28B912 498A15D34965D227B1 31C9 4C3A75B854BA79A2 74 6813B81A32 6C91B8C9AD2 57

$\pi_{3}(14,5)=(169,169,165,156,155,153,151,147,142,137,133,124,109,75,16,1)$. norm $=290288$

964E73E574DB518381AB23D415CD4B1A7B898A76C34D2B69EE132A6CAC65218B 9A359C1218449B35 ADE 62AC62747B28569714453327DBCA3AD 571BE2889B 658126BAB929C685A4C2 76AC1 988F $9345 \mathrm{~B} 73$ 65 6A9A 8E 3727245A8E2B11D2B3C 9B 75451783941BD8AD 7287736142B5CE 6838352A14DCD 1C 85B 463 E6EAC97A89121EBA6392529A64B72DB1629A7C5483ED8C5275D973EC8A6916C85425C1684 4D 91372 AEB37CD 41293ED2 41A538E8 19ED 5A6DB 7539168AA4 912BDBC1879B 7234953482DD3967CAE 14157 AE 371 A $3514126 \mathrm{FA} 3 \mathrm{~B} 7 \mathrm{C} 2664 \mathrm{AB} 9834 \mathrm{C} 8 \mathrm{C} 5 \mathrm{D} 2 \mathrm{~B} 46516 \mathrm{D} 7 \mathrm{AC} 539 \mathrm{~B} 26 \mathrm{~B} 72 \mathrm{C} 982143856 \mathrm{DA} 18 \mathrm{C} 7 \mathrm{~A} 5 \mathrm{E} 62 \mathrm{~B} 37468 \mathrm{~A}$ 3D17C491326974C51B5623482E7215C4A59D67599B886C3A84D7543727DB3634C16D8192ED172B58 EDB485A 9C4DA5A71C7D2 932C3491C3754925A3E6321B781928A 461E1B75BC828311D4DC58B6734C4 62A 97B6CB235419A 54E197A63E 39628586CA7394515B17291D68B 453C2238696A 4B 49681 3CB29D38 5A12B75C123584945AAB73CCA617235DBE68D1BB7EA672C4D 636895D 759CA12382A 9B1475B3C7213 45694251D928235C14A7C6D1DE96C817926938DA756361D94B2 45A587B2C84476398614B67BAD 227 43C3A49771C6531B82B5E6DC3729184E1893DA7A16D34E1D3B1687AB8CB658E4 42939C2A 54E3DBF2 8C4195275DA1 638ADB2 96C184531D2 4B5A6372A5BCA4B 9615F 847 E65B8BC31 38C153C4A221B 76 B 38 93251DAC47E97A6CB5597DA13B271856A62 485C49C562E1422C597B68DA32D67B84A517369C384E 7 1D324BC51762B8DE 31 9E 7AAC8B1 66948759231A7389B 4654C3B2F 32E16D1285A 4A3 9C967B965C2D6 BE 185762994813 ABD 74563 FEDC172DC9AB51C37284964AD 782C 33F 2C55719E 82E 567147CB2143AEA $314928627 C 34$ 93E1B5219834C92D6615E4C92A 759D 49D 7621536F762C594 37B658E218A95384BD51 68B3DEC7A8 64 34.6D5A57B3DC9171B28319B4DA3A8BC5E1 2E24C67615C3E 9981435 C646C72 918D 565 
APPENDIX TABLE (Continued)

42A2ABE12CA 743B54D81DBA379FC6C723782164BD19846A1B5A95273D5A434C8GD218BB86371A69A 5718CB3AD 7C 481C 35EAE5F 42F 96243 B 7193482B 65712AC79D 1842673264 D 57A1697123CE325BCB 42 69D 68197A5 89AD4 3B1 7B8 965EBA8D 4452A 3EC52B1A4BE32C7136CE3A 6172B 43A9814175D268A2 191

C2852BA73DC64 9B86354AA 4782DCD 8619A75735B94 6B2 93C8D435A62137294551476C312E 9D 8A5FD

A28B6471D3EB92563E814C867A14C259D1387BC8A67B4 3B89A746951235912CEDF8AB235C61A3EB2 198552EA3C9E1 8562F 4D 43796B5DB4 328657726D 9A139D89C751421E463A782FEC1D736B9542D 482 61367B428DE6A38B9134C8A15E2 4965C337D121C94D2A75B85 4DA79A3746812B81A236F 91B8E9AC3 57

$\pi_{+}(14.5)=(169,169,163,156,155,152,149,148,142,139,132,131,102,76,19)$, norn $=289872$

B 4763834A858B2AC52D 415EC2DB86C294F 956DB4 7DA9134A772E1EB69D5A12AB346A8B2127B6B 743 679416531898F1797C32835D 419C637A496C25B1EF568A721949C51386E57A91D8C326B5EBA7469D 3C4 366B57F 17139B8D1A2 281C8AAC 74362583B42AE 9C 751A 57962E1DCD65A8 9D 41423C552BAEC 436 B8A43 9B 4 57212976E391D1 4CC3BB1 46231587A7985C9DAE1379A53B4A58A2646871 892A784962CB1 F5C6EA3821 9CBD 1328 A5945289D 3CA5B6CE 72 4368B52146792ADD3C1848AC3C1B 4D 86B7D 42523645 C629752A216B4B4E51D37CEAC57BEAB31976425BD8A393C17391468127956FD82C6537BD1357AC65 732EB942419C6869239E1B4713B129BA3AC6857D8BF64CAE85D7A43C1546A9C8625472C1862C1DA5 385B 734D9576A 8E2D641E51784924E6A7A1D83A3C12D9B24198352426774A81852274CD8945763F 3 E15B6B78313CD2D8EB62EC6B9E 9351CA75844BD3B2862A19295D9A57A11786D3B 4C6C 8426D 51C37D 54214 93A21 3D 8796E54DA63B4 92D1ACB 3785326B 956A71CE4 388B3C9594A8216518A 427D6C 37A1 29 55A9B1625C1B16672DBC3982A 7C7A 42481DBCC3857B7324E5B1373598914AF578D 89326E64BC411C DA93EA84 72C5832BE 164 E53BA51A2C682DF947D829937A2DB72F634D586457C651A9C81DB3EABD51 8432961CD7C26E4C831A6524579231B7E869C187B34D5B6259C37C64A7DB8236724A8E41125639AD 681C2B5B4 93EFC 3BADA 79B32E 4182835641 BC $9 A 57843162911$ E 4B 36735A 91 AF 85D 7762C9848D 56C 4 2A91A3A62 851 4D 76D23849784D299C5EA531D2B634859378BAC14A152632159B 47839A58BE6A71CC 87263B51E9B 724B6E3857B9D 432619EAD 8726C9154B3AE6D 71945C1BACD23B9135A 8276541278947 C2561B31AE53C5829B12A4786F1E872A6D3C1745C8D69831247EA7514AEBDC93E5A12 64D8D 9B 3432 67C5D8B83967C537EB5CE 598E2C2A1462E3B9DA4 959C821C173FEB2A546C392D 4AB39A8E13294BCB 7151C8621F 4EC75A 3862BD7756F 9AE719431286C62A4A8B23737A1C5D64D7B74A51298EC33627C8D 3926394574AB72CCEB9D8 34168A145672DAB5144C32145B87268196517B3A532786215B8B15C96A1 83D 392 4ECBAEC 9D 5827BE 5399C7BED 4616483 E 1624 3DFB13925A 4 6BD 527156C34 92B2A 781FD8 1252 B14A1BD 9934A785AC83769C3518864A276C56A59CDAE1 3B844597E312A5163AC2 6875C21487B5689 31EBD3729AEC4 1CB 43A276893C24B148324A8D 873549AB65C9D37B6215C92166C7AD61E9A 82 4BCC1 24B73169B5A326781AD 7EBCD45A566717E9C91C 46823A 454B892312345B8961E382958C669B14D51 7283A 4D17996DA7C32C473A2B51675CA4B3A21297C51C864 97DB453843E5621382D1B7CA2E85D3A6 B9

phism program NAUTY ([134], [135]). We thank him for letting us use this program.

\section{APPENDIX \\ TABIIFS (OF PAR'TILIONS}

This Appendix gives a number of partitions of the set of all $\left(\begin{array}{l}n \\ n\end{array}\right)$ binary vectors of length $n$ and wcight $w$ into disjoint codes with distance 4 (sce Section VI and especially Table VI). To explain the notation,

$$
\pi(6,3)=(4,4,4,4,2,2), \text { norm }=72: 23541.3642141 .36122543
$$

indicates that there is a partition of the 20 6-bit, weight- 3 binary words into 6 disjoint distance- 4 codes, of respective sizes $4,4,4$, 4,2 , and 2. The norm is $4^{2}+4^{2}+4^{2}+4^{2}+2^{2}+2^{2}=72$. The lexicographically first word of $\Pi(6,3)$ (namely 000111) is in code 2 , the second word (namely 001011 ) is in code 3 , the third is in code $5 . \cdots$ and the 20th word (namely 111000 ) is in code 3 .

The digit-string giving the code-numbers (or "colors") is in a constant-width font with 80 characters per line, and the colors are represented by $1,2,3, \cdots, 9, \mathrm{~A}, \mathrm{~B}, \mathrm{C}, \cdots$.
REFERF.NCIS

[1] E. F. Assmus. Jr. and J. D. Key, "On an infinite class of Steiner systems with $t=3$ and $k=6, " J$. Combin. Theory, vol. 42A, pp. $55-6 \mathbf{0}, 1986$

[2] E. F. Assmus, Jr. and H. F. Mattson, Jr., "Disjoint Steiner systems associated with the Mathieu groups," Bull. Amer. Math Soc., vol. 72, pp. 843-845, 1966.

[3] __ "New 5-designs," J. Combin. Theon, vol. 6, pp. 122-151. 1969

[4] R. D. Baker, "Partitioning the planes of $A G_{2 m}$ (2) into 2-dcsigns," Discrete Math., vol. 15. pp. 205-211, 1976.

[5] R. D. Baker, J. H. van Lint, and R. M. Wilson, "On the Preparata and Guethals codes," IEEE Trans. Inform. Theory, vol. IT-29, pp. 342-345, 1983.

[6] E. Balas, V. Chvátal, and J. Nešetřil, "On the maximum weighted clique problem," Math. Oper. Res., vol. 12, pp. 522-535, 1987.

[7] E. Balas and C. S. Yu. "Finding a maximal clique in an arbitrary graph,” SIAM J. Comput ., vol. 15, pp. 1054-1068, 1986.

[8] L. D. Baumert, "Cyclic difference sets," Lecture Notes Math, vol 182. New York: Springer-Verlag, 1971.

[9] S. Bays. "Une question de Cayley relative au problème des triades de Steiner," Enseignement Math., vol. 19, pp. 57-67, 1917.

[10] C. Berge, " $\Lambda$ new color change to improve the coloring of a graph." Discrete Appl. Math., vol. 24, pp. 25-28, 1989.

[11] M. R. Best, " $A(11,4,4)=35$ or some new optimal constant weight codcs," Math. Centr. Report ZN 71/77, Amsterdam, Feb. 1977 
[12] __, "Binary codes with a minimum distance of four," IEEE Trans. Inform. Theory, vol. IT-26, pp. 738-742, 1980.

[13] A. E. Brouwer, F. J. MacWilliams, A. M. Odlyzko, and N. J. A. Sloane, "Bounds for binary codes of length less than $25, "$ IFEE Trans. Inform. Theory, vol. I'I-24, pp. 81-93, 1978.

[14] T. Beth, D. Jungnickel, and H. Lenz, Design Theory. New York: Cambridge University Press, 1986.

[15] M. Bläum. "A $(16,9,6,5,4)$ error-correcting dc free block code," ILLE Trans. Inform. Theory, vol. 34, no. 1, pp. 138-141. Jan. 1988

[16] B. Bose and T. R. N. Rao, "Theory of unidirectional error correcting/detecting codes," IEEE Trans. Comput., vol. 31, pp. $521-530,1982$

[17] R. C. Bose and S. Chowla, "Theorems in the additive thcory of numbers," Comment. Math. Heliet., vol. 37, pp. 141-147, $1962-63$

[18] R. C. Bose and W. S. Connor, "Combinatorial properties of group divisible incomplete block designs," Ann. Math. Stat., vol. 23, pp. 367-383, 1952.

[19] D. R. Breach and $\Lambda$. P. Street, "Partitioning sets of quadruples into designs II," J. Comb. Math. Comb. Computing, vol. 3, pp. $41-48,1988$

[20] A. E. Brouwer, " $A(17,6,4)=20$ or the nonexistence of the scarce design $S D(4.1 ; 17,21)$." Math. Centr. Report ZW62, Amsterdam, Dec. 1975 .

[21] . "On the packing of quadruples without common triples," Ars. Comb., vol. 5, pp. 3-6, 1978

[22] _ .Optimal packings of $K_{4}$ 's into a $K_{n}, " J$. Comb. Theory vol. 26A, pp. 278-297, 1979.

[2.3] _ "A f cw new constant weight codes," IEEE Trans. Inform. Theory, vol. 26, p. 366, 1980

[24] "Some unitals on 28 points and their embeddings in projective planes of order 9, , in Geumetries and Groups. M. Aigner and D. Jungnickel, Eds., Lect. Notes Math., vol. 893. New York: Springer-Verlag, 1981, pp. 183-188.

[25] "Partial linear spaces with one point and one line more than affine planes-Report on results of K. Metsch as communicated by A. Blokhuis." unpublished manuscript, 1989.

[26] "A unital in the Hughes plane of order nine", preprint.

[27] - "Block designs," Discrete Math.. vol. 77, pp. 55-56. 1989

[28] A. E. Brouwer, P. Delsarte and P. Piret, "On the $(23,14,5)$ Wagner code," IEEE Trans. Inform. Theory, vol. 26, pp. 742-743, 1980.

[29] A. E. Brouwer, A. Schrijver, and H. Hanani, "Group divisible designs with block-size four," Discrete Math., vol. 20, pp. 1-10 1977.

[30] P. J. Cameron. Parallelisms of Complete Designs. New York: Carmb. Univ. Press, 1976.

[31] P. J. Cameron and J. H. van Lint, Graphs, Codes and Designs. New York: Camb. Univ. Press, 1980.

[32] A. Cayley, "On the triadic arrangements of seven and fifteen things," Phil. May, vol. 37. pp. 50-53. 1850; Collected Mathematical Papers, vol. I, pp. 481-484

[33] Y. Chen, "The Steiner system $S(3,6,26)$, , J. Cienmetry, vol. 2, pp $7-22,1972$.

[34] Z. Chen, F. Jin, and P. Fan, "On a new hinary $(22,13,5)$ code," IEEE Trans. Inform. Theory, vol. 36, no. 1, pp. 228-229, Jan 1990.

[35] W. R. Cheswick, personal communication.

[36] L. G. Chouinard II, "Partitions of the 4-subsets of a 13-set into disjoint projective planes," Discrete Math., vol. 45, pp. 297-300, 1983.

[37] H. Chung and P. V. Kumar, "Optical orthogonal codes-New bounds and an optimal construction," IEEE Trans. Inform. The on'. vol. 36, no. 4, July 1990

[38] F. N. Cole, L. D. Cummings, and HI. S White, "The complete enumeration of triad systems in 15 clcments," Proc. Nat. Acad. Sci. USA, vol. 3, 1917, pp. 197-199.

[39] S. D. Constantin and T. R. N. Rao "On the theory of binary asymmetric error correcting codes," Inform. Contr., vol. 40, pp. $2036,1979$.

[40] J. H. Conway, "The miracle octad gencrator," in Topics in Group Theory and Computation, M. P. J. Curran, Ed. New York: Academic Press, 1977, pp. 62-68.

[41] J. H. Conway, R. T. Curtis. S. P. Norton, R. A. Parker, and R. A. Wilson. ATLAS of Finite Groups. New York: Oxford Univ. Press, 1985.
[42] J. H. Conway, R. A. Parker, and N. J. A. Sloane, "The covering radius of the Leech lattice," Proc. Roy. Soc. Lon., vol. 380A, pp. 261-291, 1982.

[43] J. H. Conway and N. I. A. Sloanc, "Laminated lattices," Ann. Math., vol. 116, pp. 593-620, 1982.

[44] "Lexicographic codes: Error-correcting codes from game theory," IEEE Trans. Inform. Theon, vol. 32, pp. 337-348, 1986.

[45] __, Sphere Packings, Iattices and Groups. New York: Springer-Verlag, 1988.

[46] _ "Orbit and coset analysis of the Golay and related codes," IEEE Trans. Inform. Theory, vol. 36, no. 5, pp. 1038-1050, Sept. 1990.

[47] R. T. Curtis, "On subgroups of -0. 1. Lattice stabilizers," J. Alg., vol. 27, pp. 549-573, 1973 .

[48] _. "A new combinatorial approach to $M_{24}$," Math. Proc. Camb. Phil. Soc., vol. 79, pp. 25-42, 1976.

149] N. Darwish and B. Bose, "New lower hounds for constant weight and asymmetric error correcting codes," preprint

150] P. Delsarte and P. Piret, "Spectral cnumcrators for certain additive-error-correcting codes over intcger alphahets," Inform. Contr., vol. 48. pp. 193-210, 1981.

[51] __ "Bounds and constructions for binary asymmetric errorcorrecting codes," IEEE Trans. Inform. Theon, vol. 27, pp. 125-128, 1981; correction, vol. 36. no. 4, p. 954, July 1990.

[52] P. Dembowski, Finite Geometries. Berlin: Springer-Verlag, 1968

[53] R. H. F. Denniston, "Some maximal arcs in finite projective planes," J. Combin. Theory, vol. 6, pp. 317-319, 1969

[54] . "Some packings with Steiner triple systems," Discrete

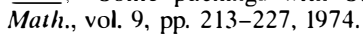

[55] __ "Sylvester's problem of the 15 schoolgirls," Discrete Math. vol. 9 , pp. 229-233, 1974

[56] __ "Some new 5-designs." Bull. Lond. Math. Soc., vol. 8, pp. 263-267, 1976 .

[57] __ "Four doubly resolvable completc 3-dcsigns," Ars. Comb., vol. 7 , pp. $265-272,1979$

[58] __ , "Enumeration of symmetric designs $(25,9.3)$, , Ann. Discrete Math.. vol. 15, pp. 111-127. 1982.

[59] I. Diener, "On cyclic Steiner systems S(3.4.22)," pp. 301-313 of [128].

[60] J. Doyen, "Constructions of disjoint Steiner triple systems," Proc. Amer. Math. Soc., vol. 32, pp. 409 416, 1972.

[61] J. Doyen and A. Rosa, "An updated bibliography and survcy of Steiner systems," Ann. Discrete Math., vol. 7, pp. 317-349, 1980.

[62] __ "A bibliography and survey of Steiner systems," preprint, Feb. 1989.

[63] G. Dueck and T. Scheuer, "Threshold accepting: A general purpose optimization algorithm appearing superior to simulated annealing," preprint.

[64] T. Etzion, "Constructions of error-correcting DC-free block codes," IEEE Trans. Inform. Theory, vol. 36, no. 4, pp. 889-905, July 1990

[65] __ "Optimal partitions for triples," J. Comb. Theon, to appear.

[66] , personal communication

[67] T. Etzion and A. Hartman, "Towards a large set of Steiner quadruplc systems," SIAM J. Discrete Math., to appear

[68] T. Etzion and C. L. M. van Pul, "New lower bounds for constant weight codcs," IEEE Trans. Inform. The ry, vol. 35. no. 6, pp. 1324-1329, Nov 1989.

[69] A. A. El Gamal, L. A. Ilemachandra, I. Shperling, and V. K. Wei, "Using simulated annealing to design good codes," IEEE Trans. Inform. Theory, vol. IT-33, pp. 116-123, 1987

[70] L. Gerhards and W. Lindenberg, "Clique detection for nondirected graphs: Two new algorithms." Computing, vol. 21, pp 295-322, 1979.

[71] M. Goldberg and T. Spencer, "A new parallel algorithm for the maximal independent set problem," SLAM J. Comput., vol. 18 pp. $419-427,1989$.

[72] R. L. Graham and N. J. A. Sloane, "Lower bounds for constant weight codes," IEEE Trans. Inform. Theory, vol. IT-26, pp. $37-43,1980$.

[73] __ "On additive bases and harmonious graphs," SIAM J. Alg. Discrete Methods, vol. 1, pp. 382-404, 1980.

[74] M. J. Grannell and T. I. Griggs, "A note on the Steiner systems S(5, 6, 24)," Ars. Comb., vol. 8, pp. 45-48, 1979.

[75] R. K. Guy. Unsolved Problems in Number Theon. New York: Springer-Verlag, 1981

176] H. Halberstam and K. F. Roth, Sequences. New York: 
Springer-Verlag, 1966

[77] M. Hall, Jr.. Combinatorial Theory. Watham, MA: Blaisdell 1967.

[78] M. Hall, Jr. and J. D. Swift. "Determination of Stcincr triple systems of order 15," Math. Tables Aids Comput., vol. ๆ. pp. 146-152, 1955.

[79] H. Hämäläinen, "Two new binary codes with minimum distance three," IEEE Trans. Inform. Theory, vol. 34, p. 875, 1988.

[80] , personal communication.

[81] H. Hanani, "On quadruple systems," Canad. I. Math., vol. 12 , pp. 145-157, 1960 .

[82] __ "The existence and construction of halanced incomplete block designs," Ann. Math. Stat., vol. 32, pp. 361-386, 1961

[83] ___ " "A balanced incomplete block dcsign," Ann. Math. Stat., vol. 36, p. 711,1965

[84] __ "Balanced incomplete block designs and related designs," Discrete Math., vol. 11, pp. 255-369, 1975.

[85] A. Hartman, "The existence of rcsolvable Steiner quadruple systems," J. Comb. Theory, vol. 44A, pp. 182-206, 1987.

[86] A. Hartman, W. H. Mills, and R. C. Mullin, "Covering triples by (juadruples: An asymptotic solution," J. Combin. Theory, vol. 41A, pp. $117-138,1986$

[87] A. A. Hashim and A. G. Constantinides, "Some new results on binary linear block codes," Electron. Lett., vol. 10, pp. 31-33, 1974

[88] A. A. Hashim and V. S. Pozdniakov, "Computerized search for binary linear codes," Electron. Lett., vol. 12, pp. 350-351, 1976

[89] H. J. Helgert and R. D. Stinaff, "Minimum-distance bounds for binary linear codes," IEEE Trans. Inform. Theory. vol. 19, pp. $344-356,1973$

[90] 1. Helleseth and T. Kløve, "On group theoretic codes for asymmetric channels," Inform. Contr., vol. 49, pp. 1-9, 1981.

[91] I. Honkala, "Lower hounds for constant weight codes," (in Finnish), Master's dissertation. Mathematics Dept., Univ. Turku, Turku, Finland, Jan. 1985.

[92] __ "Some optimal constant weight codes," Ars. Comb., vol. 20B, pp. 43-47, 1985.

[93] personal communication.

[94] I. Honkala. H. Hämäläinen, and M. K. Kaikkonen, "A modification of the Zinoviev lower bound for constant weight codes," Discrete Appl. Math., vol. 11. pp. 307-310, 1985

[95] _ "Some lower bounds for constant weight codes," Discrete Appl. Math., vol. 18, pp. 95-98, 1987.

[96] D. R. Hughes and F. C. Piper, Design Theory. Cambridge: Cambridge Univ. Press, 1985.

[97] B. Hupert, Endliche Gruppen I. New York: Springer-Verlag, 1967

[98] D. S. Johnson, C. R. Aragon, L. A. McGeoch, and C. Schevon, "Optimization by simulated annealing: An experimental evaluation. Part 1,” Oper. Res., vol. 37, pp. 865-892, 1989.

[99] S. M. Johnson. "A new upper bound for error-correcting codes," IEEE Trans. Inform. Theory, vol. IT-8, pp. 203-207, 1962.

[100] __ "Improved asymptotic bounds for error-correcting codes," IEEE Trans. Inform. Theory, vol. IT-9, pp. 198-205, 1963.

[101] "On upper bounds for unrestricted binary error-correcting codes," IEEE Trans. Inform. Theory, vol. IT-17, pp. 466-478, 1971

[102] _ "Upper bounds for constant weight error correcting codes," Discrete Math., vol. 3, pp. 109-124, 1972.

[103] G. A. Kabatiansky and V. I. Panchenko, "Packings and coverings of Hamming space with unit spheres," (in Russian). Probl. Peredach. Inform., vol. 24, no. 4, pp. 3-16, 1988

[104] M. K. Kaikkonen, "A new four-error correcting code of length 20," IEEE Truns. Inform. Theory, vol. 35, no. 6, p. 1344, Nov. 1989.

[105] , personal communication.

[106] J. G. Kalbfleisch and R. G. Stanton, "Maximal and minimal coverings of $(k-1)$-tuples by $k$-tuples," Pac. J. Math., vol. 26, pp. 131-140, 1968

[107] W. M. Kantor, "On the inequivalence of generalized Preparata codes," IEEE Trans. Inform. Theory, vol. IT-29, pp. 345-348, 1983.

[108] R. E. Kibler, "Some new constant weight codes," IEEE Trans. Inform. Theory, vol. 26, pp. 364-365, 1980

[109] T. P. Kirkman, "On a problem in combinations," Cambridge and Dublin Math. J., vol. 2, pp. 191-204, 1847.

[110] T. Klyve, "A lower bound for $A(n, 4, w)$," IEEE Trans. Inform Theory, vol. 27, pp. 257-258, 1981
[111] K.-U. Koschnick, "On the construction of some new constant weight codes." preprint.

[112] A Kotzig and J. Turgeon, "On certain constructions for Latin squares with no subsquares of order two," Discrete Math., vol. 16, pp. 263-270, 1976.

[113] E. S. Kramer, "Some triple system partitions for prime powers," Utilitas Math., vol. 12, pp. 113-116, 1977.

[114] E. S. Kramer and S. S. Magliveras, "Some mutually disjoint Steiner systems," J. Combin. Theory, vol. 17A, pp. 39-43, 1974.

[115] E. S. Kramer and D. M. Mesner, "Intersections among Steiner systems," J. Combin. Theory, vol. 16A, pp. 273-285, 1974.

[116] , "The possible (impossible) systems of 11 disjoint $\boldsymbol{S}(2,3,13)$ 's $(S(3,4,14)$ 's $)$," Utilitas Math., vol. 7, pp. 55-58, 1975

[117] "Admissible parameters for Steincr systems $S(t, k, l)$ with a table for all $(l-t) \leq 498$," Unlitas Math., vol. 7, pp. 211-222. 1975

[118] _ " $t$-Designs on hypergraphs," Discrete Math., vol. 15, pp 263-296, 1976.

[119] D. L. Kreher and S. P. Radziszowski, "The cxistence of simple 6-(14,7,4) designs," J. Combin. Theon, vol. 43A, pp. 237--243, 1986

[120] C. W. II. Lam, L. Thiel, and S. Swicrcz. "The non-existence of finite projectivc plancs of order 10," Canad. J. Math., vol. 41, pp. 1117-1123, 1989.

[121] H. Lenz, "Tripling Stciner quadruple systems," Ars. Combin., vol. 20. pp. 193-202, 1985.

[122] V. I. Levenshtein, "The application of Hadamard matrices to a problem in coding." Probl. Kihernetiki, vol. 5, pp. 123-136, 1961. English translation in Probl. Cybern., vol. 5, pp. 166-184, 1961.

[123] Shen Lin, personal communication.

[124] C. C. Lindner, "A notc on disjoint Steiner quadruple systems," Ars. Combin., vol. 3, pp. 271-276, 1977.

[125] _ "On the construction of pairwise disjoint Steiner quadruplc systems," Ars. Combin., vol. 19, pp. 153-156, 1985.

[126] C. C. Lindner and A. Rosa, "There are at least 31,021 non-isomorphic Steiner quadruple systems of order 16," Utilitas Math. vol. 10, pp. 61-64, 1976.

[127] _ "Steiner quadruple systems - $\Lambda$ survey," Discrete Math., vol. 22, pp. 147-181, 1978.

[128] C. C. Lindner and A. Rosa, Eds., "Topics on Steiner systems," Annals of Discrete Mathmatics, vol. 7. Amsterdam: North-Holland, 1980.

[129] J. H. van Lint, "A new description of the Nadler code," IEEE Trans. Inform. Theory, vol. IT-18, pp. 825-826, 1972.

[130] I. X. Lu, "On large sets of disjoint Steiner triple systems I-VI," J. Combin. Theory, vol. 34A, pp. 140-183, 1983; vol. 37A, pp. $136-192,1984$.

[131] _ _ "On large sets of disjoint Steiner triple systems VII," unfinished manuscript.

[132] F. J. MacWilliams and N. J. A. Sloane, The Theory of Error-Correcting Codes. Amsterdam: North-Holland, 1979.

[133] R. Mathon and A. Rosa, "Tables of parameters of BIBDs with $r \leq 41$ including existence, enumeration, and resolvability results." Ann. Discrete Math., vol. 26, pp. 275-308, 1985.

[134] B. D. McKay, “NAUTY user's guide (version 1.2),” Dept. Comput. Sci. Tech. Rep. TR-CS-87-03, Australian Nat. Univ., Canberra, Australia, 1987.

[135] _ _NAUTY notes," preprint, Feb. 2, 1989.

[136] E. Mendelsohn and A. Rosa, "One-factorizations of the com plete graph-A survey,” J. Graph Theory, vol. 9. pp. 43-65, 1985.

[137] N. S. Mendelsohn and S. H. Y. Hung, "On the Steiner systems $S(3,4,14)$ and $S(4,5,15)$," Utilitas Math., vol. 1, pp. 5-95, 1972.

[138] W. H. Mills, "A new 5-design," Ars. Combin., vol. 6, pp. 193-195, 1978.

[139] R. C. Mullin and A. Rosa, "Orthogonal Steiner systems and generalized Room squares," in Proc. 6th Manitoba Conf. Num. Math., Winnipeg, 1976. Congr. Numer. XVIII, Util. Math. Publ. Inc., Winnipeg, 1977, pp. 319-323.

[140] P. M. Pardalos and G. P. Rodgers, " $\Lambda$ branch and bound algorithm for the maximum clique problem," preprint.

[141] I. Peterson, Search yields math proof no one can check," Science, vol. 134 , p. 406 Dec. 1988.

[142] K. T. Phelps, "On cyclic Steiner quadruple systems $S(3,4,20)$," pp. $277-300$ of [128].

[143] "A class of large sets of Steiner triple systems of order 15," preprint.

[144] _ "A class of 2-chromatic SQS(22)," preprint

[145] $\longrightarrow$ personal communication. 
[146] K. T. Phelps and A. Rosa, "2-chromatic Steiner quadruple systems," Europ. J. Combin., vol. 1, pp. 253-258, 1980.

[147] P. Piret, "Good linear codes of length 27 and 28," IEEE Trans. Inform. Theory, vol. IT-26, p. 227, 1980

[148] V. Pless. "The children of the $(32,16)$ doubly even codes," IEEE Trans. Inform. Theory, vol. IT-24, pp. 738-746, 1982.

[149] C. L. M van Pul. "On bounds on codes," M.Sc. dissertation, Dept. of Math. and Comput. Sci., Eindhoven Univ. of Technul., Aug. 1982

[150] "Some distance problems in coding theory," Ph.D. dissertation, Dept. of Math. and Comput. Sci., Eindhoven Univ. of Technol., 1987.

[151] S. Rankinen, personal communication.

[152] D. K. Ray-Chaudhuri and R. M. Wilson, "Solution of Kirkman's schoolgirl problem," in Combinatorics, T. S. Motzkin, Ed., Proc. Symp. Pure Math.. vol. 19, pp. 187-203, 1971.

[153] M. Rciss, "Ubcr cine Steinersche Combinatorische Aufgabe wclchc im 45sten Bande dieses Journals. Seite 181, gestellt worden ist," J. Reine Angew. Math., vol. 56, pp. 326-344, 1859.

[154] V. Rödl, "On a packing and covering problem." Lurop. J. Cornhin., vol. 6, pp. 69-78, 1985.

[155] A. M. Romanov, "Ncw binary codes with minimal distance three," (in Russian), Probl. Peredach. Inform., vol. 19, no. 3, pp. 101-102, 1983.

[156] A. Rosa. R. A. Mathon, and K. T. Phelps, "Small Steiner triple systems and their properties," Ars. Combin., vol. 15, pp. 3-110, 1983.

[157] J. Schönheim, “On maximal systems of $k$-tuples," Studia Scient. Math. Hungar., vol. 1, pp. 363-368, 1966.

[158] S. Schreiber. "Covering all triples on $n$ marks by disjoint Steiner systems," J. Combin. Theory, vol. 15A, pp. 347-350, 1973.

[159] N. V. Semakov and V. A. Zinoviev, "Complete and quasi-complete balanced codes," (in Russian), Probl. Peredach. Inform., vol. 5, no. 2, pp. 14-18, 1969. English translation in Probl. Inform. Transm.. vol. 5, no. 2, pp. 11-13, 1969.

[160] "Balanced codes and tactical configurations," (in Russian), Probl. Peredach. Inform., vol. 5, no. 3, pp. 28-37, 1969. English translation in Probl. Inform. Transm., vol. 5, no. 3, pp. 22-28, 1969.

[161] M. J. Sharry and A. P. Street, "Partitioning sets of triples into designs I," Ars. Combin., vol. 26B, pp. 51-66, 1988.

[162] "Partitioning sets of triples into designs II," J. Combin. Math. Combin. Computing, vol. 4, pp. 53-68, 1988.

[163] S. S. Shrikhande, "Affine resolvable balanced incomplete block designs: A survey," Aequat. Math., vol. 14, pp. 251-269, 1976.

$|164|$ N. J. A. Sloane, S. M. Reddy, and C. L. Chen, "New binary codes," IEEE Trans. Inform. Theory, vol. IT-18, pp. 503-510, 1972.

[165] N. J. A. Sloane and J. J. Seidel, "A new family of codes obtained from conference matrices," Ann. NY Acad. Sci., vol. 175 Article 1, pp. 363-365, 1970.

[166] N. J. A. Sloane and D. S. Whitehead, "A new family of singleerror correcting codes," IEEE Trans. Inform. Theory, vol. IT-16, pp. 717-719, 1970.

[167] Warren D. Smith and N. J. A. Sloane, "Bounds for spherical codes," preprint.

[168] R. G. Stanton and I. P. Goulden, "Graph factorization, general triple systems, and cyclic triple systems," Acquat. Math., vol. 22 pp. $1-28,1981$.

[169] R. G. Stanton and M. J. Rogers, "Packings and coverings by triples," Ars. Combin., vol. 13, pp. 61-69, 1982.

[170] D. Stinson, "Determination of a packing number," Ars. Combin. vol. 3, pp. 89-114, 1977.

[171] L. Teirlinck, "On the maximum number of disjoint Steiner triple systems," Discrete Math., vol. 6, pp. 299-300, 1973.

[172] "On making two Steiner triple systems disjoint," J. Combin. Theory, vol. 23A, pp. 349-350, 1977.

[173] _ "On large sets of disjoint ordered designs," Ars. Combin. vol. 25 , pp. $31-37,1988$.

[174] _ "A complction of Lu's determination of the spectrum for large sets of disjoint Stciner triple systems," preprint.

[175] H. C. A. van Tilborg and M. Blaum, "On error-correcting balanced codes," IEEE Trans. Inform. Theory, vol. 35, no. 5, pp. 1091-1095, Sept. 1989

[176] V. D. Tonchev, Combinatorial Configurations, Designs, Codes, Graphs. New York: Longman, Harlow, 1988.

[177] T. Verhoeff, "An updated table of minimum-distance bounds for binary linear codes," IEEE Trans. Inform. Theory. vol. IT-33, pp. 665-680, 1987.

[178] _ _ "An updated table of minimum-distance bounds for binary linear codes," (revised), preprint, Jan. 1989.

[179] T. J. Wagner, "A search technique for quasi-perfect codes," Inform. Contr., vol. 9, pp. 94-99, 1966.

[180] J. H. Weber, C. de Vroedt, and D. E. Boekee, "Bounds and constructions for binary codes of length less than 24 and asymmetric distance less than 6," IEEE Trans. Inform. Theory, vol. 34 pp. $1.321-1.331,1988$

[181] R. M. Wilson, "Some partitions of all triples into Steiner triple systems," in Hypergraph Seminar, Ohio State Univ., 1972: Lecture Notes Math., vol. 411. New York: Springer-Verlag, 1974 pp. $267-277$

[182] E. Witt, "Die 5-fach transitiven Gruppen von Mathieu," Abh. Math. Sem. Univ. Hamburg, vol. 12, pp. 256-264, 1938.

[183] . "Ueber Steinersche Systeme," loc. cit., vol. 12, pp. 265-275, 1938.

[184] A. Zaptcioglu, "Entwicklung störsicherer Binärcodes," Diplomarbeit, Lehrstuhl für Informatik. Technische Universitat Braunschweig, Germany, Aug. 6, 1981.

[185] L. Zhu, "A pair of orthogonal Steiner systems $S(3,5,17)$," Discrete Math., vol. 31, pp. 111 113,1980.

[186] V. A. Zinoviev, "Cascade equal-weight codes and maximal packings." Probl. Contr. and Inform. Theory, vol. 12, no 1, pp. 3-10, 1983.

[187] "On a generalization of the Johnson bound for constantweight codes," (in Russian), Probl. Peredach. Inform., vol. 20, no. 3, pp. 105-108, 1984.

[188] V. A. Zinoviev and S. N. Litsyn, "On recurrent relations for the cardinality of equal-weight codes," in Proc. Internat. Workshor Algebraic and Combin. Coding Theory, Varna, Bulgaria, 1988, pp $181-183$. 